LA W RENCE LIVERMORE NATIO NAL LABORATORY

\section{Energy Spread Reduction of Electron Beams Produced via Laser Wake}

B. Pollock

April 3, 2012 
This document was prepared as an account of work sponsored by an agency of the United States government. Neither the United States government nor Lawrence Livermore National Security, LLC, nor any of their employees makes any warranty, expressed or implied, or assumes any legal liability or responsibility for the accuracy, completeness, or usefulness of any information, apparatus, product, or process disclosed, or represents that its use would not infringe privately owned rights. Reference herein to any specific commercial product, process, or service by trade name, trademark, manufacturer, or otherwise does not necessarily constitute or imply its endorsement, recommendation, or favoring by the United States government or Lawrence Livermore National Security, LLC. The views and opinions of authors expressed herein do not necessarily state or reflect those of the United States government or Lawrence Livermore National Security, LLC, and shall not be used for advertising or product endorsement purposes.

This work performed under the auspices of the U.S. Department of Energy by Lawrence Livermore National Laboratory under Contract DE-AC52-07NA27344. 


\title{
UNIVERSITY OF CALIFORNIA, SAN DIEGO
}

\section{Energy Spread Reduction of Electron Beams Produced via Laser Wakefield Acceleration}

\author{
A dissertation submitted in partial satisfaction of the \\ requirements for the degree \\ Doctor of Philosophy \\ in \\ Engineering Science (Engineering Physics) \\ by \\ Bradley Bolt Pollock
}

Committee in charge:

Professor George Tynan, Chair

Professor Farhat Beg

Professor Tom O'Neil

Professor Kevin Quest

Professor Mark Tillack 
Copyright

Bradley Bolt Pollock, 2012

All rights reserved. 
The dissertation of Bradley Bolt Pollock is approved, and it is acceptable in quality and form for publication on microfilm and electronically:

\section{Chair}

University of California, San Diego

2012 


\section{DEDICATION}

To my father, by whose example I have lived my entire life. To my mother, who has always been there for me. To Robin, who never stopped believing in me. 
EPIGRAPH

Excellence does not

require perfection.

-Henry James 


\section{TABLE OF CONTENTS}

Signature Page ......................... iii

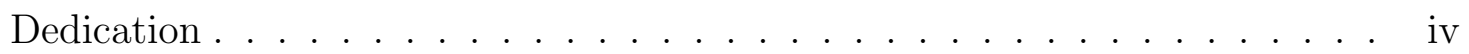

Epigraph .......................... . $\mathrm{v}$

Table of Contents ......................... vi

List of Figures . . . . . . . . . . . . . . . . . . viii

List of Tables ......................... . . . . . . . . . . .

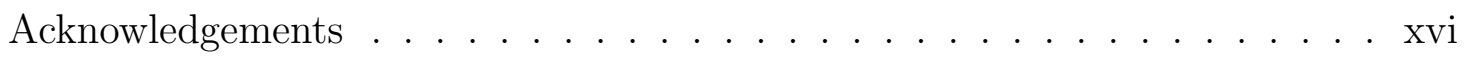

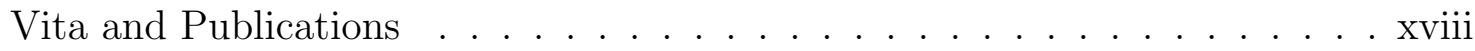

Abstract of the Dissertation .................. . xxi

Chapter 1 Introduction . . . . . . . . . . . . . . . . . . 1

1.1 Brief History of Laser Wakefield Acceleration . . . . . . . 1

1.2 Summary of Results . . . . . . . . . . . . . . . . . 5

1.3 Outline ..................... 5

Chapter 2 Physics of Laser Wakefield Acceleration . . . . . . . . . . . . . 6

2.1 Electron Blowout by the Laser Ponderomotive Force . . . 6

2.1.1 The Ponderomotive force . . . . . . . . . . 7

2.1.2 Self-guiding of the laser pulse . . . . . . . . 8

2.2 The Wake Electric Field . . . . . . . . . . . . . . . . . 10

2.3 Electron Injection and Trapping in the Wake . . . . . . . 15

2.3.1 Self-injection............ . 15

2.3.2 Ionization-induced Injection . . . . . . . . . . 17

2.4 Energy Gain from LWFA . . . . . . . . . . . . . . 18

2.4.1 Dephasing and Pump Depletion . . . . . . . . 18

2.4.2 Dephasing-limited energy gain . . . . . . . . 22

Chapter $3 \quad$ Experimental Setup .................. 24

3.1 Callisto Laser System . . . . . . . . . . . . . . . . . . 24

3.2 General Experimental Setup of the Callisto Target Cham-

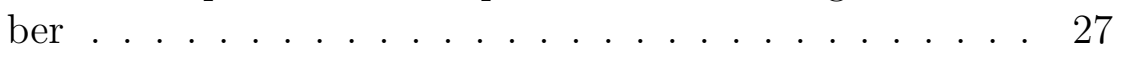

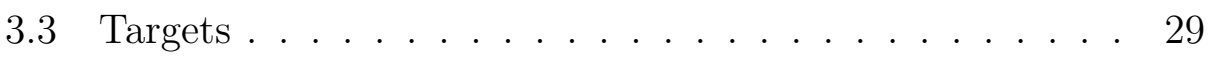

3.3.1 Gas Jets . . . . . . . . . . . . . . . . . . 29

3.3.2 Gas Cells ................ . . 29 
3.4 Diagnostics . . . . . . . . . . . . . . . . . . 29

3.4.1 Electron Spectrometer . . . . . . . . . . 30

3.4 .2 Interferometry . . . . . . . . . . . . . . . . . . . 33

3.4 .3 Spectroscopy . . . . . . . . . . . . . . 36

3.4.4 Transmitted Laser Spot Imaging . . . . . . . . . . 37

3.4.5 Transmitted Laser Spectrum . . . . . . . . . . . 37

Chapter 4 Plasma Length Scaling and Self-Trapping Threshold . . . . . . 39

4.1 Electron Energy Gain Over a General Accelerating Length 42

4.2 Gas Jets . . . . . . . . . . . . . . . . . . 43

4.3 Evidence of Wake Formation . . . . . . . . . . . . 47

4.4 Electron Energy Scaling . . . . . . . . . . . . . . . 49

4.5 Trapping Threshold . . . . . . . . . . . . . . . 56

Chapter $5 \quad 1.45 \mathrm{GeV}$ Electrons from Ionization-Induced Injection . . . . . 58

5.1 Gas Cell Target . . . . . . . . . . . . . . . 58

5.2 Ionization-induced Injection Mechanism . . . . . . . . . 61

5.3 Experimental Results . . . . . . . . . . . . . . 66

Chapter 6 Electron Beam Energy Spread Reduction by Limiting the IonizationInduced Injection Region . . . . . . . . . . . . . . . . 70

6.1 Consequences of non-uniformities in the longitudinal density profile . . . . . . . . . . . . . 71

6.2 Two-stage LWFA experiment . . . . . . . . . . . . 74

Chapter 7 Conclusions . . . . . . . . . . . . . . . . . 81

7.1 Future Work . . . . . . . . . . . . . . . . 82

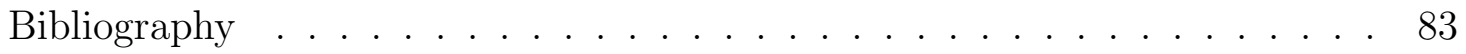




\section{LIST OF FIGURES}

Figure 2.1: A focused, $\mathrm{a}_{0}>2,60$ fs laser pulse (in red) is shown propagating to the right, where the frame is moving with the laser pulse. At the rising edge of the pulse the ponderomotive force "blows out" electrons (trajectories shown in black), while the stationary ions (not shown) provide a restoring space-charge force. Behind the laser pulse electrons are pulled back toward the laser axis and overshoot due to their inertia, and the resulting plasma wave wake has a radially directed electric field as described in the text. Along the laser propagation axis this field is purely longitudinal and is the accelerating force for LWFA. For a given plasma density and laser power, Eq. 2.15 determines the radius $\mathrm{R}$ of the blown-out region. The focusing geometry of the laser should be chosen such that the spot size $\mathrm{w}_{0} \approx \mathrm{R}$ in order to satisfy the matching condition and achieve stable laser guiding in the plasma. . . . . . . . . . . . . . .

Figure 2.2: The longitudinal electric field profile (the field along the laser axis) of the first bucket is shown, where the localized spike at the back of the bucket is due to the locally high electron density arising from electron trajectory overlap at the laser axis. The width and depth of the spike are sensitive to the exact trajectories of the blown-out electrons, and in the simulations of $\mathrm{Lu}$ et. al.[1] the amplitude is roughly 3 times the linear maximum value. . . . . . . . . . . . . . . . . . . .

Figure 2.3: The electron sheath at the back of the first bucket and the front of the second bucket are shown (in black), along with the trajectory of a self-trapped electron (in red). The trapped electron's trajectory intersected the high electric field region at the back of the first bucket such that the field pushed the electron forward to a sufficient energy for it to travel at the wake phase velocity. . . . . . . . . . . . . 16

Figure 2.4: As the laser propagates it continuously loses energy to the wake via red-shifting of laser photons. As photons red-shift they move more slowly in the underdense plasma, and the head of the laser pulse erodes backward. The left panel shows the initial laser pulse before any pump depletion, while the center and right panels show increasing amounts of pump depletion corresponding to increased laser propagation distances in the plasma. The front of the laser pulse moves backward in the plasma at the etching rate $v_{\text {etch }}=c n_{e} / n_{c} \ldots \ldots \ldots$. . . . . . 
Figure 2.5: Electrons injected at the back of the laser pulse are in a region of accelerating field. Due to the spherical symmetry of the ions in the wake, once the electrons have moved past the center of the wake the sign of the electric field reverses as shown in Fig. 2.2. Electrons that propagate through this portion of the wake lose energy in the decelerating electric field. Electrons traverse the accelerating field region over a laboratory distance of one dephasing length. . . . . . . . . . . . . . . .

Figure 3.1: Block diagram of the main components of the Callisto Laser System. . . . . . . . . . . . . . . 25

Figure 3.2: Typical stages of a CPA laser system. . . . . . . . . . . . . . . 26

Figure 3.3: The otical layout inside the Callisto target chamber. The main laser pulse and the interferometer probe pulse are shown, as well as the interferometry and spectroscopy optical layouts. The probe pulse is split from the main pulse at the output of the 5-pass amplifier and compressed to 100 fs duration with a secondary compressor. After traversing the target the laser pulse is partially reflected by a $5 \mu \mathrm{m}$ pellicle, while the remaining laser light is dumped on a $100 \mu \mathrm{m} \mathrm{Al} \mathrm{light} \mathrm{shield} \mathrm{at} \mathrm{the} \mathrm{target}$ chamber wall. Electrons pass through the pellicle and the light shield to enter the dipole magnet outside the vacuum chamber. The reflected laser light is collimated before leaving the chamber and is directed to optical diagnostics that measure the spot size and spectrum of the beam after traversing the plasma. . . . 28

Figure 3.4: The 200 TW Callisto laser is focused by an $\mathrm{f} / 8$ off-axis parabola to a vacuum spot size of $\mathrm{w}_{0}=15$ microns at the leading edge of a gas target. The target delivers He gas at neutral densities of $1 \times 10^{18}-1 \times 10^{19} \mathrm{~cm}^{-3}$. A dipole magnet is placed after the target, followed by two image plates. The displayed trajectories through the magnet correspond to the features labeled B1 and B2 on the image plates. . . . . . . . . . . . . .

Figure 3.5: a) The energy of each of our electron beams is measured first using both image plates and then again with just the front image plate. The solid line divides electron beams with positive (below the line) and negative (above the line) deflection angles. b) The deflection angles for all electron beams are shown; there is no apparent correlation to beam energy. . . . . . . . . . . . . . 34

Figure 3.6: Typical inteferogram (in false color) recorded during the twostage gas cell experiments with the corresponding Abel-inverted density profile. . . . . . . . . . . . . . . 35 
Figure 4.1: Shown on the left are electrons trapped at the back of the first bucket of the wake at a radius $\mathrm{R}$ from the center of the wake. The laser has been omitted in this figure, but propagates to the right inside the wake (as in Fig. 2.1). The trapping is assumed here to occur at the start of the plasma, corresponding to a laser propagation length $l=0$ in the plasma. The electrons propagate to the right at essentially c, while the the wake (and laser) propagates at the reduced speed $\mathrm{v}_{\phi}$ determined by Eq. 2.24. On the right is the case corresponding to the laser having propagated one dephasing length in the plasma, where the electrons have now reached the position $\mathrm{r}=0$ and the zero of the longitudinal electric field (see Fig. 2.2). . . . . . . . . . . . .

Figure 4.2: The energy gain calculated by Eq. 4.4 is normalized to the dephasing limited energy gain from Eq. 2.26 and plotted against the acceleration length normalized to the dephasing length. When $l_{a c c}=0$ the electron is at the back of the bucket. As the laser propagates the electron moves toward the center of the bucket; after one dephasing length in the laboratory frame the electron has moved from the back of the bucket to the center of the bucket and the zero of the longitudinal electric field. If the plasma is longer than a dephasing length the laser continues to propagate and the electron moves into the decelerating electric field portion of the wake. If an electron moved all the way to the front of the wake, corresponding to the laser propagating two dephasing lengths in the plasma, the net energy gain for the electron goes to zero for a symmetric electric field. . . . . .

Figure 4.3: The alignment technique used to align the laser to the gas jets is shown schematically. The target chamber has been brought to a partial vacuum of 10 Torr of air. As the $250 \mathrm{~mJ}$ alignment laser (with a Gaussian transverse intensity profile) focuses the intensity on axis eventually reaches the ionization threshold for the nitrogen and oxygen molecules in the air. This gives an initially small plasma region defined by the volume of the laser pulse that is sufficiently intense to ionize the air. As the laser continues to focus the ionization radius from the laser axis becomes larger, until the whole pulse is above the ionization intensity; after this point the ionized region follows the focusing laser profile. Downstream of the focal plane the process occurs in the reverse order, and the resulting two-lobe structure has a minimum in the center corresponding to the laser focal plane. . 44

Figure 4.4: Photograph of the $5 \mathrm{~mm}$ diameter nozzle gas jet. The $3 \mathrm{~mm}$ and $8 \mathrm{~mm}$ nozzle are of similar design. . . . . . . . . . . 
Figure 4.5: Characteristic electron density profiles for the $3 \mathrm{~mm}, 5 \mathrm{~mm}$, and $8 \mathrm{~mm}$ diameter gas jets. The backing pressure for each case is given. . . . . . . . . . . . . . . . . . . 4 46

Figure 4.6: Laser light reflected by the pellicle inside the target chamber is collimated by a lens before exiting the target chamber. The collimated light is partially reflected by a beamsplitter into the exit spot imaging system, while the transmission through the beamsplitter is directed into the transmitted spectrum system. The spot size diagnostic consists of a foucsing lens that creates a 1:1 image of the plasma exit, which is magnified by a $10 \mathrm{x}$ microscope objective onto a 14-bit CCD camera. The system yields $0.7 \mu \mathrm{m} /$ pixel magnification with a resolution of $\approx 20 \mu \mathrm{m}$. The spectral diagnostic also creates a 1:1 image of the plasma exit plane, but here at the entrance slit to a $1 / 3-\mathrm{m}$ spectrometer that is coupled to a 16-bit CCD camera. This spectral dispersion is $0.27 \mathrm{~nm} /$ pixel and has a spectral field of view of 280 nm. . . . . . . . . . . . . . . . .

Figure 4.7: Raw dispersed electron spectra from the first image plate are shown for the maximum electron energy achieved in each of the gas jets. The laser power in each case is $65 \mathrm{TW}$. . . . . . . . . 50

Figure 4.8: The dephasing length calculated in Eq. 2.25 for a laser power of $65 \mathrm{TW}$ is shown in red. The black squares correspond to the electron density which maximized the electron energy for each gas jet length. . . . . . . . . . . . . . . . . 51

Figure 4.9: The dephasing-limited energy gain calculated in Eq. 2.26 for a laser power of $65 \mathrm{TW}$ is shown in red. In addition, the black squares indicate maximum energy achieved in each gas jet at the corresponding electron density. . . . . . . . . . . . 52

Figure 4.10: The dephasing length calculated in Eq. 2.25 for a laser power of $65 \mathrm{TW}$ is shown in red. The black squares indicate the useful length of the plasma for each gas jet length at the density corresponding to the maximum recorded electron energy. . . . . 53

Figure 4.11: Eq. 4.4 is plotted with the experimental data corresponding to the maximum observed electron energy from each gas jet. . . . 54

Figure 4.12: The charge observed on all high-power system shots. The vertical dashed line at $2.5 \times 10^{18} \mathrm{~cm}^{-3}$ represents the lowest density where reliable self-injection is observed in these experiments. . . 56 
Figure 5.1: A photograph of the gas cell target. The aluminum base mounts to a set of motorized translation stages which allow the gas cell to be positioned under vacuum. The body is made of polycarbonate to allow an optical axis transverse to the laser axis for interferometry. An aluminum plate is attached to the front (back) of the polycarbonate body to seal the end of the cell; this plate contains a $500 \mu \mathrm{m}(2 \mathrm{~mm})$ aperture for the laser to propagate through the cell and for the electrons to exit the cell. The gas supply line is built into the aluminum base and the polycarbonate body, where a rubber gasket with a hole is placed between the two to form a gas seal. . . . . . . . . . . . . . 59

Figure 5.2: The front aperture of the gas cell is used as a knife-edge to align the gas cell. . . . . . . . . . . . . . . .

Figure 5.3: The temporal profile of a 60 fs laser pulse with a peak $\mathrm{a}_{0}=3.5$ is shown with the ionization locations for He (black circles) and O (red circles) electrons. . . . . . . . . . . . . . . 62

Figure 5.4: The trapping process for K-shell O electrons born inside the wake. 63 Figure 5.5: An electron ionized at a radius $\mathrm{r}=\mathrm{R}_{i}$ from the center of the wake does not start gaining energy from the wake until it reaches the position $\mathrm{r}=-\mathrm{R}_{i}$. The electron can then only become trapped if the remaining potential available is sufficient for the electron to reach the phase velocity of the wake before arriving at the back of the bucket (at radius $-\mathrm{R}) \ldots \ldots \ldots \ldots$. . . . . . .

Figure 5.6: Electron spectrum from a $\mathrm{P}=110 \mathrm{TW}$ experiment with an electron density of $1.3 \times 10^{18} \mathrm{~cm}^{-3}$. The peak energy is $1.45 \mathrm{GeV}$, but the energy spread is quasi-continuous due to the method of injection. . . . . . . . . . . . . . .

Figure 6.1: Schematic representation of the effects of a density step in a LWFA gas target. The laser propagates a distance $\mathrm{z} 1$ in the target at density $\mathrm{n} 1$, forming a blown-out ion cavity with radius R1. Electrons trapped at the rear of the cavity (and at the beginning of the target) have traveled a distance R1-b in the cavity when the laser reaches $\mathrm{z} 1$ in the target (the case $\mathrm{z} 1=\mathrm{L}$ corresponds to electrons reaching the cavity center, where $\mathrm{b}=0$, and dephasing). a) The density is reduced at $z 1$, causing the cavity radius to increase to $\mathrm{R} 2$ and the cavity center to shift toward the electrons. b) The density increases at z1, causing the blow-out radius to decrease to $\mathrm{R} 2$ and the cavity center to shift away from the electrons. . . . . . . . . . . . . . 72 
Figure 6.2: Schematic of the experimental setup showing the $800 \mathrm{~nm}$ Callisto laser beam (in red), the two-stage gas cell, the $800 \mathrm{~nm}$ probe beam (in blue), a measured interferogram with its associated Abel inverted density profile (above the interferometer CCD camera), the plasma emission spectrometer and CCD camera, plasma emission images (shown inside the gas cell windows) with the spatially resolved plasma emission spectrum along the laser axis from each stage of the gas cell, the vacuum laser axis after the gas cell (red dashed line), the 0.42T dipole magnet (20 $\mathrm{cm}$ long, centered $66 \mathrm{~cm}$ from the exit of the gas cell), the deflected electron trajectory (green dashed line) onto the image plates (located $132 \mathrm{~cm}$ and $192 \mathrm{~cm}$ from the exit of the gas cell), and the optical path of the transmitted laser light (in grey) to an imaging system and a prism spectrometer. The transverse size of the plasma observed in the interferogram is larger in the accelerator section because the non-coupled (and therefore unguided) diffracting laser light ionizes a volume larger than the sub-50 $\mu \mathrm{m}$ wake, which is not resolved by this diagnostic. Optical access for the transverse diagnostics is provided by constructing the walls of the gas cell from microscope slides pressed against rubber gaskets to form a seal. The source of the Si line in the plasma emission image is suspected to be minute amounts of Si out-gassing from the gaskets under vacuum. The gas cell entrance (exit) aperture is $0.5(2.0) \mathrm{mm}$. . . . . . . . . . .

Figure 6.3: a) Magnetically dispersed electron beam images from a $4 \mathrm{~mm}$ injector only gas cell (top) and the $8 \mathrm{~mm}$ two-stage cell (bottom). b) Electron spectra above $70 \mathrm{MeV}$ for: the $8 \mathrm{~mm}$ two-stage injector-accelerator cell (dotted blue curve) filled to an electron density of $3 \times 10^{18} \mathrm{~cm}^{-3}$ in each stage for a coupled laser power of $40 \mathrm{TW}$; the $4 \mathrm{~mm}$ injector-only cell (solid red curve) filled to an electron density of $3.4 \times 10^{18} \mathrm{~cm}^{-3}$ for a coupled laser power of $50 \mathrm{TW}$. The injector gas fill in each case is $99.5 \% \mathrm{He}$ and $0.5 \%$ $\mathrm{N}_{2}$, and the total charge is indicated for each spectrum. c) The total observed charge above $70 \mathrm{MeV}$ for injector gas fills of pure He (red squares) and $99.5 \%$ He with $0.5 \% \mathrm{~N}_{2}$ (blue circles) for coupled laser powers between 30-60 TW. . . . . . . . . . . 76 
Figure 6.4: a,b) Images of the transmitted laser light at the exit of the 4 $\mathrm{mm}$ injector stage and the $8 \mathrm{~mm}$ two-stage gas cell, respectively. Vertically integrated lineouts indicate the guided laser spot size, while the vacuum laser spot size at the exit of the cell is denoted for each case by a yellow circle. A long-pass filter is placed in front of the camera to attenuate unshifted and blue-shifted light. c,d) The transmitted laser spectrum from the injector only and the two-stage cell, respectively. A mask is used to block the fundamental $800 \mathrm{~nm}$ light to take better advantage of the dynamic range of the 8-bit, frame grabbed, infrared camera. The spectral fringes are due to the etalon effect of the uncoated pellicle beamsplitter $\left(5 \mu \mathrm{m}\right.$ thick at $\left.45^{\circ}\right)$ seen in Fig.6.2. For each spectrum the recorded false-color image is shown. . . . . 78 


\section{LIST OF TABLES}

Table 5.1: The ionization potentials and corresponding the $\mathrm{a}_{0}$ required to ionize as determined from ADK tunnel ionization[2] for He and O. 63 


\section{ACKNOWLEDGEMENTS}

There are a great many who deserve my thanks for their efforts in getting me to where I am today. In particular I would like to thank Dr. Dustin Froula for being the outstanding mentor that he was, especially during the early part of my graduate career. My thanks also to Dr. Siegfried Glenzer for his leadership and constant push to be better. And finally a thanks to Prof. George Tynan for all of the advice and fresh perspective provided throughout my studies. Without these three individuals I would not have pursued a graduate degree.

Additionally I would like to acknowledge the support that I have received from Prof. Chan Joshi's group at UCLA both in conducting experiments at Callisto and in running the OSIRIS simulations of the experiments. The laser crew at the Jupiter Laser facility also deserves recognition for their support of the Callisto experiments.

This work was performed under the auspices of the Department of Energy by the Lawrence Livermore National Laboratory under Contract No. DE-AC5207NA27344.

Chapter 3, in part, is a reprint of the material as it appears in: B. B. Pollock, J.S. Ross, G. R. Tynan, D. H. Froula, S. H. Glenzer, V. Leurent, J. P. Palastro, J. E. Ralph, C. E. Clayton, K. A. Marsh, A. E. Pak, T. L. Wang, and C. Joshi. TwoScreen Method for Determining Electron Beam Energy and Deflection from a Laser Wakefield Accelerator. Proceedings of the 2009 Paricle Accelerator Conference, Vancouver, 2009(http://epaper.kek.jp/PAC2009/papers/we6rfp101.pdf), p. A14. The dissertation author was the primary investigator and author of this paper.

Chapter 6, in part, is a reprint of the material as it appears in: B.B. Pollock, G.R. Tynan, F. Albert, C. Filip, S.H. Glenzer, J. Meinecke, A. Pak, J.E. Ralph, C.E. Clayton, C. Joshi, K.A. Marsh, J. Shaw, K.L. Herpoldt, and D.H. Froula. The effects of a density mismatch in a two-stage LWFA. Proceedings of the 2011 Paricle Accelerator Conference, New York, 2011 (http://www.cad.bnl.gov/pac2011/proceedings/papers/weobs3.pdf), p. 1. The dissertation author was the primary investigator and author of this paper.

Chapter 6, in part, is a reprint of the material as it appears in: B. B. Pollock, 
C.E. Clayton, J.E. Ralph, F. Albert, A. Davidson, L. Divol, C. Filip, S.H. Glenzer, K. Herpoldt, W. Lu, K. Marsh, J. Meinecke, W.B. Mori, A. Pak, T.C. Rensink, J.S. Ross, J. Shaw, G.R. Tynan, C. Joshi, and D.H. Froula. Demonstration of a narrow energy spread, $0.5 \mathrm{GeV}$ electron beam from a two-stage Laser Wakefield Accelerator. Phys. Rev. Lett., 107, 045001 (2011). The dissertation author was the primary investigator and author of this paper. 


\section{VITA}

2006

2007

2007-2009

2008-2012

2012
B. S. in Engineering Physics cum laude, University of the Pacific

Graduate Teaching Assistant, University of California, San Diego

Graduate Student Researcher, University of California, San Diego

Lawrence Scholar, Lawrence Livermore National Laboratory

Ph. D. in Engineering Physics, University of California, San Diego

\section{PUBLICATIONS}

B.B. Pollock, D.H. Froula, P.F. Davis, J.S. Ross, S. Fulkerson, J. Bower, J. Satariano, D. Price, K. Krushelnick, and S.H. Glenzer, "High magnetic field generation for laser-plasma experiments", Rev. Sci. Instr., 77, 114703 (2006).

B.B. Pollock, D.H. Froula, L. Divol, D. Price, R. Costa, F. Yepiz, S.H. Glenzer, and G.R. Tynan, "Multi-cm Long High Density Magnetic Plasmas for Optical Guiding", Rev. Sci. Instr., 79, 10F550 (2008).

B.B. Pollock, P. Davis, L. Divol, S.H. Glenzer, J.P. Palastro, D. Price, G.R. Tynan, and D.H. Froula, "Magnetically Controlled Optical Plasma Waveguide for Electron Acceleration", Proceedings of the Thirteenth Advanced Accelerator Concepts Workshop, Santa Cruz, CA, 2008 (AIP, New York, 2009), pp. 165-170.

B.B. Pollock, J.S. Ross, G.R. Tynan, D.H. Froula, S.H. Glenzer, V. Leurent, J.P. Palastro, J.E. Ralph, C.E. Clayton, K.A. Marsh, A.E. Pak, T.L. Wang, and C. Joshi, "Two-Screen Method for Determining Electron Beam Energy and Deflection from a Laser Wakefield Accelerator", Proceedings of the 2009 Paricle Accelerator Conference, Vancouver, 2009 (http://epaper.kek.jp/PAC2009/papers/we6rfp101.pdf), p. A14.

D.H. Froula, J.S. Ross, B.B. Pollock, P. Davis, A.N. James, L. Divol, M.J. Edwards, A.A. Offenberger, D. Price, R.P.J. Town, G.R. Tynan, and S.H. Glenzer, "Quenching of the nonlocal electron heat transport by large external magnetic fields in a laser produced plasma measured with imaging Thomson scattering", Phys. Rev. Lett., 98, 135001 (2007). 
J.S. Ross, S.H. Glenzer, J.P. Palastro, B.B. Pollock, D. Price, L. Divol, G.R. Tynan, and D.H. Froula, "Observation of Relativistic Effects in Collective Thomson Scattering", Phys. Rev. Lett., 104, 105001 (2010).

D.H. Froula, L. Divol, P. Davis, J.P. Palastro, P. Michel, V. Leurent, S.H. Glenzer, B.B. Pollock, and G. Tynan, "Magnetically controlled plasma waveguide for laser wakefield acceleration", Plasma Phys. Control. Fusion, 51, 024009 (2009).

D.H. Froula, C.E. Clayton, T. Doppner, K.A. Marsh, C.P.J. Barty, L. Divol, R.A. Fonseca, S.H. Glenzer, C. Joshi, W. Lu, S. Martins, P. Michel, W. Mori, J.P. Palastro, B.B. Pollock, A. Pak, J.E. Ralph, J.S. Ross, C.W. Siders, L.O. Silva, and T. Wang, "Measurements of the critical power for self-injection of electrons in a laser wakefield accelerator", Phys. Rev. Lett., 103, 215006 (2009).

V. Leurent, P. Michel, C.E. Clayton, L. Divol, T. Doppner, S.H. Glenzer, C. Joshi, K.A. Marsk, A. Pak, J.P. Palastro, B.B. Pollock, J. Ralph, G.R. Tynan, T. Wang, and D.H. Froula, "Study of x-ray radiation from a laser wakefield accelerator", Proceedings of the Thirteenth Advanced Accelerator Concepts Workshop, Santa Cruz, CA, 2008 (AIP, New York, 2009), pp. 235-240.

D.H. Froula, L. Divol, N. Meezan, S. Dixit, J. Moody, P. Neumayer, B.B. Pollock, J.S. Ross, and S.H. Glenzer, "Ideal laser beam propagation through high temperature ignition hohlraum plasmas", Phy. Rev. Lett., 85, 085001 (2007).

D.H. Froula, L. Divol, N.B. Meezan, S. Dixit, P. Neumayer, J.D. Moody, B.B. Pollock, J.S. Ross, L. Suter, and S.H. Glenzer, "Laser beam propagation through inertial confinement fusion hohlraum plasmas", Phys. Plasmas, 14, 055705 (2007).

D.H. Froula, V. Rekow, C. Source, K. Piston, R. Knight, S. Alvarez, R. Griffith, D. Hargrove, J.S. Ross, S. Dixit, B.B. PollockL. Divol, S.H. Glenzer, W. Armstrong, R. Bahr, K. Thorp, and G. Pien, "3 omega transmitted Beam diagnostic at the Omage Laser Facility", Rev. Sci. Instr., 77, 10E507 (2006).

A. Visco, R.P. Drake, D.H. Froula, S.H. Glenzer, and B.B. Pollock, "Temporal dispersion of a spectrometer", Rev. Sci. Instr., 79, 10F545 (2008).

J.P. Palastro, J.S. Ross, B. Pollock, L. Divol, D.H. Froula, and S.H. Glenzer, "Fully Relativistic Form Factor for Thomson Scattering", Phys. Rev. E., 81, 036411 (2010).

J.E. Ralph, C.E. Clayton, F. Albert, B.B. Pollock, S.F. Martins, A.E. Pak, K.A. Marsh, J.L. Shaw, A. Till, J.P. Palastro, W. Lu, S.H. Glenzer, L.O. Silva, W.B. Mori, C. Joshi, and D.H. Froula, "Laser wakefield acceleration at reduced density in the self-guided regime", Phys. Plasmas, 17, 056709 (2009). 
C.E. Clayton, J.E. Ralph, F. Albert, R.A. Fonseca, S.H. Glenzer, C. Joshi, W. Lu, K.A. Marsh, S.F. Martins, W.B. Mori, A. Pak, F.S. Tsung, B.B. Pollock, J.S. Ross, L.O. Silva, and D.H. Froula, "Self-Guided Laser Wakefield Acceleration beyond 1 GeV Using Ionization-Induced Injection", Phys. Rev. Lett., 105, 105003 (2010).

J.S. Ross, J.L. Kline,S. Yang, M. Henesian, T. Weiland, D. Price, B.B. Pollock, and S.H. Glenzer, "4 omega Thomson scattering probe for high-density plasma characterization at Titan", Rev. Sci. Instr., 81, 10D524 (2010).

J.S. Ross, S.H. Glenzer, J.P. Palastro, B.B. Pollock, D. Price, G.R. Tynan, and D.H. Froula, "Thomson-scattering measurements in the collective and noncollective regimes in laser produced plasmas", Rev. Sci. Instr., 81, 10D523 (2010).

B.B. Pollock, G.R. Tynan, F. Albert, C. Filip, S.H. Glenzer, J. Meinecke, A. Pak, J.E. Ralph, C.E. Clayton, C. Joshi, K.A. Marsh, J. Shaw, K.L. Herpoldt, and D.H. Froula, "The effects of a density mismatch in a two-stage LWFA", Proceedings of the 2011 Paricle Accelerator Conference, New York, 2011 (http://www.cad.bnl.gov/pac2011/proceedings/papers/weobs3.pdf), p. 1.

B.B. Pollock, C.E. Clayton, J.E. Ralph, F. Albert, A. Davidson, L. Divol, C. Filip, S.H. Glenzer, K. Herpoldt, W. Lu, K. Marsh, J. Meinecke, W.B. Mori, A. Pak, T.C. Rensink, J.S. Ross, J. Shaw, G.R. Tynan, C. Joshi, and D.H. Froula, "Demonstration of a narrow energy spread, $\sim 0.5 \mathrm{GeV}$ electron beam from a twostage Laser Wakefield Accelerator", Phys. Rev. Lett., 107, 045001 (2011). 


\title{
ABSTRACT OF THE DISSERTATION
}

\section{Energy Spread Reduction of Electron Beams Produced via Laser Wakefield Acceleration}

\author{
by \\ Bradley Bolt Pollock \\ Doctor of Philosophy in Engineering Science (Engineering Physics) \\ University of California, San Diego, 2012 \\ Professor George Tynan, Chair
}

\begin{abstract}
Laser wakefield acceleration of electrons holds great promise for producing ultra-compact stages of $\mathrm{GeV}$ scale, high quality electron beams for applications such as x-ray free electron lasers and high energy colliders. Ultra-high intensity laser pulses can be self-guided by relativistic plasma waves over tens of vacuum diffraction lengths, to give $>1 \mathrm{GeV}$ energy in cm-scale low density plasma using ionization-induced injection to inject charge into the wake at low densities.

This thesis describes a series of experiments which investigates the physics of LWFA in the self-guided blowout regime. Beginning with high density gas jet experiments the scaling of the LWFA-produced electron beam energy with plasma electron density is found to be in excellent agreement with both phenomenological
\end{abstract}


theory and with 3-D PIC simulations. It is also determined that self-trapping of background electrons into the wake exhibits a threshold as a function of the electron density, and at the densities required to produce electron beams with energies exceeding $1 \mathrm{GeV}$ a different mechanism is required to trap charge into low density wakes.

By introducing small concentrations of high-Z gas to the nominal He background the ionization-induced injection mechanism is enabled. Electron trapping is observed at densities as low as $1.3 \times 10^{18} \mathrm{~cm}^{-3}$ in a gas cell target, and $1.45 \mathrm{GeV}$ electrons are demonstrated for the first time from LWFA. This is currently the highest electron energy ever produced from LWFA. The ionization-induced trapping mechanism is also shown to generate quasi-continuous electron beam energies, which is undesirable for accelerator applications. By limiting the region over which ionization-induced trapping occurs, the energy spread of the electron beams can be controlled. The development of a novel two-stage gas cell target provides the capability to tailor the gas composition in the longitudinal direction, and confine the trapping process to occur only in a limited, defined region. Using this technique a $460 \mathrm{MeV}$ electron beam was produced with an energy spread of 5\%. This technique is directly scalable to multi-GeV electron beam generation with sub-percent energy spreads. 


\section{Chapter 1}

\section{Introduction}

\subsection{Brief History of Laser Wakefield Accelera- tion}

The acceleration of beams of electrons to high energies is of great importance throughout the scientific community. In addition to particle physics studies of collisions between electrons and positrons, high energy electron beams can be used to produce high energy beams of x-rays (such as in the case of x-ray free-

electron lasers and betatron radiation sources). Conventional linear acceleration of electrons using radio-frequency $(\mathrm{RF})$ oscillating electric fields has produced electron beams with energies up to $50 \mathrm{GeV}$ at the Stanford Linear Accelerator (SLAC, the longest linear accelerator in the world), with very good electron beam quality (low energy spread, low emittance, high charge)[3]. However, due to constraints on the maximum amplitude of the electric field in these RF devices (10's of $\mathrm{MeV} / \mathrm{m}$ ), the length required to achieve such high energies is several kilometers (SLAC is $3.2 \mathrm{~km}$ in length).

Alternatively, it is possible to accelerate electrons using laser-driven relativistic plasma waves with electric fields $10^{3}-10^{4}$ times greater than those achievable in RF accelerators. This process, called Laser Wakefield Acceleration (LWFA), allows for a substantial reduction in the size (and therefore cost) of an accelerator capable of producing high energy electron beams. The concept was first proposed 
in 1979 by Tajima and Dawson[4], where they predicted that the necessary electron plasma wave could be driven by an intense $\left(\mathrm{I}>10^{18} \mathrm{~W} / \mathrm{cm}^{2}\right)$, short duration $\left(\tau_{\text {Laser }} \sim \lambda_{p} / c\right)$ laser pulse to produce the accelerating structure for electrons.

The physical processes involved are charge separation induced by the the laser ponderomotive force and charge restoration due to the space charge force from the charge separation. Assuming a bi-Gaussian laser pulse (Gaussian transverse spatial profile and longitudinal temporal profile) incident on an initially quasineutral plasma (or a neutral gas such as He which is tunnel ionized many e-foldings ahead of the $\mathrm{I}>10^{18} \mathrm{~W} / \mathrm{cm}^{2}$ peak of the laser pulse), the ponderomotive force on the electrons scales as the gradient of the laser intensity and acts to move them outward from the center of the laser beam. The ions, however, are not able to respond to the high-frequency fluctuations of the laser electric field due to their inertia and so remain fixed. The maximum distance an electron travels from the laser axis is determined by balancing the space charge force of the stationary ions with the ponderomotive force from the laser. After the laser pulse passes a given location the electrons are pulled back toward the laser axis by the space charge force of the ions and overshoot; this oscillation is the desired plasma wave, or the wake. Now consider a frame moving with the phase velocity of the wake, which is nearly equal to the laser group velocity in the plasma; in such a frame the wake is stationary and the first period has an electric field structure that is positive outward from the center of the ion-only region. If an electron is now trapped at the back of this wake period (trapping mechanisms are discussed in the next chapter) it experiences a $\sim 100 \mathrm{GeV} / \mathrm{m}$ electric field in the direction of the laser propagation. The maximum energy an electron gains from this process and other limitations, such as maintaining the laser intensity over distances many times the Rayleigh length, are discussed in detail in Ch. 2.

This process can be driven directly by the laser only if the pulse duration $\tau_{\text {Laser }}$ is short enough to be contained within one period of the wake, which for electron densities $n_{e}$ of $10^{18}-10^{20} \mathrm{~cm}^{-3}$ corresponds to laser pulse lengths of $110-10$ fs. However, when LWFA was first proposed the available laser pulse lengths were $>3$ orders of magnitude too long to drive the wake directly; fortunately, beating of 
two co-propagating laser pulses with a frequency difference of a plasma frequency (beat-wave LWFA, or BWLWFA) provided a platform for early theoretical and experimental work[5]. The first demonstration of a driven wake in this regime was published in 1985[6], but it was not until 1993 that electrons were successfully injected into, trapped, and accelerated by the wake[7]. The beat-wave LWFA regime is technically challenging because it requires a two-frequency laser system, and the frequency difference must be exactly matched to the plasma frequency in order to drive the wake. The peak electron energies obtained during these early experiments were $\sim 30 \mathrm{MeV}[8]$, and required external electrons to be injected into the wake.

At nearly the same time that the beat-wave experiments were beginning to demonstrate electron acceleration, chirped-pulse amplification[9] allowed for the production of sub-ps laser pulses with terawatts of power. While the pulse lengths were still too long to excite wakefields directly, the laser intensity was now high enough to drive the forward Raman scattering instability, which in turn was able to produce the plasma waves desired for LWFA studies. This became known as the self-modulated LWFA (SMLWFA) regime, and as it offered several advantages over the beat-wave scheme (most notably the ability to use a single frequency laser) it replaced BWLWFA for most of the field. Exponential electron spectra extending to nearly $100 \mathrm{MeV}$ were produced for the first time using this technique[10], and the electric field was deduced to be more than $150 \mathrm{GeV} / \mathrm{m}$ (compared with less than $10 \mathrm{GeV} / \mathrm{m}$ from BWLWFA). Another significant advantage to this regime was the observation of self-trapping of background electrons, attributed to the large amplitude of the wakes produced by SMLWFA, in contrast to the need for external injection in BWLWFA.

Further advances in laser technology eventually led to the development of Ti:Sapphire laser systems, where pulse lengths below 100 fs and powers above 10 TW were finally realized and wakefields could be excited directly by the laser pulse. This LWFA regime is divided primarily into two categories by the intensity of the laser. The linear regime corresponds to weakly relativistic laser intensities of $\sim 10^{18} \mathrm{~W} / \mathrm{cm}^{2}$, while the so-called "blowout" regime is accessed at intensities of 
$10^{19}-10^{20} \mathrm{~W} / \mathrm{cm}^{2}$. While the scalings for energy gain and associated phenomena scale slightly differently between the two regimes, the primary difference comes with respect to guiding the laser pulse over centimeter-scale distances, which is necessary to maintain peak laser intensity longer than a Rayleigh length. The linear regime requires the use of an external guiding structure, such as a capillary or a plasma channel[11], to guide the pulse. In the blowout regime the laser pulse is self-guided by the plasma[12] (this process is discussed in Ch. 2) which reduces the complexity of the system.

Using such laser systems electron energy gains exceeding $1 \mathrm{GeV}$ have been demonstrated $[13,11]$ in the LWFA regime, with even higher energies on the horizon as higher power and higher repetition rate systems become available (such as the Texas Petawatt at University of Texas at Austin, the BELLA Laser at University of California at Berkeley and the proposed E23 at the Lawrence Livermore National Laboratory). These LWFA experiments have also made tremendous progress toward improving the quality of the electron beam, specifically in terms of energy spread. While SMLWFA beams were continuous in energy, energy spreads as low as a few percent have been reported from LWFA. One key issue for the LWFA community is the trapping of electrons into the wake as the plasma density is reduced (the motivation for which is increased energy gain, which is discussed in detail in Ch. 2). At the densities required for multi-GeV energy gain the self-trapping mechanism that was so advantageous breaks down[14]. Alternative injection schemes relying on ionization of higher-Z gases (ionization-induced injection) $[15,16]$ than the traditional $\mathrm{H}_{2}$ or $\mathrm{He}$ plasmas have been shown to be effective at densities several factors below the self-trapping threshold, but have generally led to large energy spread electron beams reminiscent of SMLWFA[13]. However, by tailoring the longitudinal gas composition of the target to limit the ionization-induced injection region, a $0.46 \mathrm{GeV}$ electron beam with 5\% energy spread has successfully been demonstrated[17]. This technique should be directly scalable to multi-GeV electron energies with presently available laser systems, and even higher energies once short-pulse laser systems advance to petawatt power capabilities. 


\subsection{Summary of Results}

This thesis presents an experimental investigation of laser wakefield acceleration in the blowout regime which produced for the first time a narrow energy spread beam of $\sim 0.5 \mathrm{GeV}$, ionization-injected electrons from LWFA[17].

We demonstrate using gas jet He targets that there is an electron density threshold for self-trapping background plasma electrons into the wake[14]. Furthermore, we show that by employing ionization-induced injection[15] this threshold can be overcome and broad spectrum electron beams with maximum energies exceeding $1 \mathrm{GeV}$ are accessible using a gas cell target[13]. Finally, by tailoring the gas composition along the gas cell we demonstrate a reduction of the energy spread of our electron beams from $50 \%$ to $5 \%$ at peak energies of $\sim 0.5 \mathrm{GeV}$.

\subsection{Outline}

The thesis is organized in the following manner: the physics of the blowout regime of LWFA is presented in Chapter 2; the experimental setup is shown in Chapter 3; the main results are discussed in Chapters 4-6; and conclusions and future work are highlighted in Chapter 7. 


\section{Chapter 2}

\section{Physics of Laser Wakefield}

\section{Acceleration}

This chapter will focus on how a high-power, short-pulse laser can be used to drive a plasma wave wake in the so-called "blowout" regime of LWFA[1]. The processes of forming the wake and laser pump depletion are presented, while the electric field structure within the wake and the electron energy gain achievable from LWFA will be calculated.

\subsection{Electron Blowout by the Laser Ponderomo- tive Force}

The blowout regime of LWFA is characterized by the normalized vector potential $a_{0}=\frac{e E}{m c \omega}=0.85 \times 10^{-9} \lambda_{\mu m} I_{W / c^{2}}^{1 / 2}$ of the laser and is valid for $a_{0}>2$. This is typically achieved by focusing short $\left(c \tau_{\text {Laser }} \sim \lambda_{p}\right)$, high power laser pulses to spot sizes of $w_{0} \sim \lambda_{p} / 2$. Here $\mathrm{c}$ is the speed of light, $\lambda_{p}=2 \pi / k_{p}=2 \pi c / \omega_{p}$, and the plasma frequency $\omega_{p}=\left(\frac{n_{e} e^{2}}{\epsilon_{0} m}\right)^{1 / 2}$. In a plasma, the ponderomotive force of the laser can be viewed as the laser radiation pressure which acts to expel electrons outward from the center of the laser beam (the inertia of the ions is too great for them to respond to the high frequency fluctuations of the laser electric field). The physical mechanism for creating the wake structure in the plasma is balancing the 
ponderomotive force from the laser with the space charge force from the ions, and for a given laser power leads to a condition relating the plasma density to the spot size of the focused laser beam.

\subsubsection{The Ponderomotive force}

For a linearly polarized laser pulse propagating in the z-direction with an electric field $\mathbf{E}=E_{0} \cos (k z-\omega t) \hat{x}$ and a corresponding magnetic field $\mathbf{B}=\frac{1}{c} E_{x} \hat{y}$ (from Maxwell's equations), the magnitude of the time-averaged Poynting vector $\langle\mathbf{S}\rangle=\frac{1}{\mu_{0}}\langle\mathbf{E} \times \mathbf{B}\rangle$ gives the laser intensity

$$
I=\frac{c \epsilon_{0}}{2} E_{0}^{2}
$$

To calculate the ponderomotive force, first rewrite the electric field in the form $\mathbf{E}=\mathbf{E}_{s} \cos (-\omega t)$, where all of the spatial information is included in $\mathbf{E}_{s}$. Newton's second law and the Lorentz force now yield the equation of motion for an electron in the laser field

$$
m \frac{d \mathbf{v}}{d t}=-e(\mathbf{E}+\mathbf{v} \times \mathbf{B})
$$

with $\mathrm{m}$ (e) the mass (charge) of the electron. The ponderomotive force is a secondorder effect, so the equation of motion must be solved for its second-order components.

Assuming the electron is initially at rest and that the only electric and magnetic fields are those of the laser (i.e. there are no dc fields present), the first order equation of motion evaluated at the initial position of the electron becomes

$$
m \frac{d \mathbf{v}_{1}}{d t}=-e \mathbf{E}\left(\mathbf{r}_{0}\right)
$$

After integrating in time the first-order velocity is $\mathbf{v}_{1}=\frac{e}{m \omega} \mathbf{E}_{s} \sin (\omega t)$, while integrating in time again gives the first-order change in position $\Delta \mathbf{r}_{1}=\frac{e}{m \omega^{2}} \mathbf{E}_{s} \cos (\omega t)$.

In second-order there is a $\mathbf{v}_{1} \times \mathbf{B}$ term in the equation of motion, where $\mathbf{B}=\frac{1}{\omega} \nabla \times \mathbf{E}_{s} \sin (\omega t)$ from Maxwell's equations. Additionally, the electric field 
expanded about the initial position of the electron is $\mathbf{E}=\mathbf{E}\left(\mathbf{r}_{0}\right)+\left(\Delta \mathbf{r}_{1} \cdot \nabla\right) \mathbf{E}(r=$ $\left.r_{0}\right)+$ higher order terms. Therefore, the second-order components are

$$
m \frac{d \mathbf{v}_{2}}{d t}=-e\left(\left(\Delta \mathbf{r}_{1} \cdot \nabla\right) \mathbf{E}+\frac{\mathbf{v}_{1}}{\omega} \times \nabla \times \mathbf{E}_{s} \sin (\omega t)\right)
$$

Substituting the first-order values of velocity and displacement into the secondorder equation of motion and taking the time average (where $\left\langle\sin ^{2}(-\omega t)\right\rangle=$ $\left.\left\langle\cos ^{2}(-\omega t)\right\rangle=1 / 2\right)$ gives

$$
\mathbf{f}_{P}=m\left\langle\frac{d \mathbf{v}_{2}}{d t}\right\rangle=-\frac{e^{2}}{2 m \omega^{2}}\left(\left(\mathbf{E}_{s} \cdot \nabla\right) \mathbf{E}_{s}+\mathbf{E}_{s} \times\left(\nabla \times \mathbf{E}_{s}\right)\right)
$$

Employing the vector identity $\mathbf{E}_{s} \times\left(\nabla \times \mathbf{E}_{s}\right)=\frac{1}{2} \nabla\left(\mathbf{E}_{s} \cdot \mathbf{E}_{s}\right)-\left(\mathbf{E}_{s} \cdot \nabla\right) \mathbf{E}_{s}$ the final ponderomotive force on a single electron is

$$
\mathbf{f}_{P}=-\frac{e^{2}}{4 m \omega^{2}} \nabla\left(E_{s}^{2}\right)=-\frac{1}{2 c n_{c}} \nabla I
$$

where $n_{c}=\frac{\omega^{2} \epsilon_{0} m}{e^{2}}$ is the critical density for the wave with frequency $\omega$.

If the intensity is now assumed to have a Gaussian transverse profile $I=$ $I_{0} e^{-2 r^{2} / w_{0}^{2}}$, where $I_{0}$ is the peak intensity on-axis and $w_{0}$ is the waist radius, the ponderomotive force is always positive outward from the beam center. Physically, an electron initially near the beam center moves from a higher field region to a lower field region in one half cycle of the laser pulse, and therefore cannot return to its initial position during the second half cycle. The ions, however, remain fixed on the time-scale of the laser pulse and provide a restoring force (calculated in Sec. 2.2) for the blown-out electrons. This process continues during successive cycles, and for $800 \mathrm{~nm}$ laser light (with a critical frequency of $1.75 \times 10^{21} \mathrm{~cm}^{-3}$ ) peak intensities of $I_{0}>0.87 \times 10^{19} \mathrm{~W} / \mathrm{cm}^{2}\left(\mathrm{a}_{0}>2\right)$ lead to a "blown-out" region void of electrons within a laser spot size $w_{0}=10-20 \mu \mathrm{m}$ from the laser axis as determined by balancing the ponderomotive and space charge forces.

\subsubsection{Self-guiding of the laser pulse}

At this point it is appropriate to introduce the concept of laser guiding in the plasma, as blowout can only occur in regions of high laser intensity. Since a 
focused laser beam will naturally diffract, the longitudinal blowout distance would be limited to a few Rayleigh lengths $z_{R}=\pi w_{0}^{2} / \lambda$ without a mechanism to guide the laser pulse at its focused spot size. One such mechanism is plasma channel guiding, where an external guiding structure is required to balance the laser diffraction with refraction in the channel. For a Gaussian laser pulse a parabolic radial density profile with a minimum on the laser axis is needed for guiding[18]. Such structures have been produced with long pulse (few nanosecond duration) lasers in low density $\left(\mathrm{n}_{e}<10^{19} \mathrm{~cm}^{-3}\right)$ plasmas using 1-5 T magnetic fields parallel to the laser axis to control heat transport and affect the radial electron density profile[19, 20, 21, 22]; simulations using the code WAKE[23] have been performed using these channel parameters and indicate that these structures are suitable for guiding a subsequent laser pulse[18]. Alternatively, channel guiding has been demonstrated in capillary discharge plasmas and has produced GeV electron beams[11]. In this scheme 200$300 \mu \mathrm{m}$ diameter capillaries are machined into sapphire blocks[24]. After filling the capillary with $\mathrm{H}_{2}$ or $\mathrm{He}$ an electrical arc discharge along the capillary axis ionizes the gas, and heat conduction to the walls forms the desired radial electron density profile to guide the short-pulse laser.

However, it has been shown recently that high power, short-pulse lasers are able to self-guide in low density plasma without the need of an external structure[12, 25].The radial index of refraction can be written in the form[26]

$$
\eta(r)=1-\frac{n_{e}}{2 n_{c}}\left(1+\frac{\Delta n_{c h}}{n_{e}} \frac{r^{2}}{w_{0}^{2}}+\frac{\Delta n}{n_{e}}-\frac{a_{0}^{2}}{8}\right)
$$

where $n_{e}$ is the background electron density and the last three terms in the parentheses are due to, respectively, external channel guiding, local density depletion from blowout, and relativistic mass corrections to the electrons. The parameter which characterizes the external channel depth is determined by solving the paraxial wave equation without the density depletion or mass corrections terms in the index of refraction and with diffraction being exactly canceled by refraction

$$
\Delta n_{c h}=\frac{4 \epsilon_{0} m c^{2}}{w_{0}^{2} e^{2}}
$$

Evaluating the channel guiding term in Eq. 2.7 at $r=w_{0}$ gives 


$$
\frac{\Delta n_{c h}}{n_{e}}=\frac{4}{k_{p}^{2} w_{0}^{2}}
$$

which is the required index of refraction to guide the laser at its minimum spot size $w_{0}$.

In the absence of a channel the same index change must be achieved from the mass correction term for self-guiding to occur. Equating these terms

$$
\frac{a_{0}^{2}}{8}=\frac{4}{k_{p}^{2} w_{0}^{2}}
$$

and using the definitions for $a_{0}, n_{c}$, and $k_{p}$ and Eq. 2.1 to relate the electric field to the intensity gives

$$
I=\frac{16}{w_{0}^{2}} \frac{n_{c} \epsilon_{0} m^{2} c^{5}}{n_{e} e^{2}}
$$

where with one final substitution relating the peak intensity of a Gaussian laser

pulse to the power $\mathrm{P}$ in the focal spot $I=\frac{2 P}{\pi w_{0}^{2}}$ the power threshold for self-guiding is

$$
P=\left(\frac{n_{c}}{n_{e}}\right) 2 m c^{3} \frac{4 \pi \epsilon_{0} m c^{2}}{e^{2}}
$$

This power is the critical power for relativistic self-focusing $\mathrm{P}_{c}=17\left(\frac{n_{c}}{n_{e}}\right) \mathrm{GW}[27]$ to occur in the plasma. If the laser power $\mathrm{P}$ exceeds this threshold, at the front of the laser pulse electrons are blown-out rapidly enough that the resulting bare ion channel can self-guide the majority of laser pulse (i.e. the laser produces its own channel in the plasma).

\subsection{The Wake Electric Field}

In addition to the ponderomotive force of the laser, electrons also experience a space-charge force from the ions they leave behind. Once the laser pulse has passed, this space-charge force acts to restore electrons to their initial positions. The electrons overshoot these positions due to their inertia, and the resulting oscillatory behavior is the plasma wave wake. This process is shown schematically 
in Fig. 2.1 in a frame moving with the laser. If the first period of the wake is assumed to be spherical, then the wake electric field in this region can be very closely approximated by Gauss's law for a sphere uniformly filled with positive charges

$$
\begin{gathered}
\oint_{S} \mathbf{E} \cdot \mathbf{d} \mathbf{A}=\int_{V}\left(\rho / \epsilon_{0}\right) d V \\
E(r)=\frac{e}{3 \epsilon_{0}} n_{e} r
\end{gathered}
$$

This radially directed electric field is purely longitudinal on the laser propagation axis, where the peak field at the back of the of the ion sphere is $E_{\max }=\frac{e}{3 \epsilon_{0}} n_{e} R$ and $\mathrm{R}$ is the blowout radius. When electron injection and trapping are discussed in the next section, it will be initially assumed that electrons injected into the wake are trapped such that their subsequent motion is along the laser axis where the radial field has no component transverse to the laser axis.

A phenomenological investigation of the blowout regime was carried out by Lu et.al.[1], and through a series of 3D particle in cell (PIC) simulations using the code OSIRIS[28] the condition relating the focused laser spot size to the electron density was found to be[1]

$$
k_{p} R \sim k_{p} w_{0}=2 \sqrt{a_{0}}
$$

where the blowout radius $\mathrm{R}$ is approximately equal to the laser spot size $w_{0}$. The above relation can be recast as

$$
a_{0}=2\left(P / P_{c}\right)^{1 / 3}
$$

for a Gaussian laser pulse. Setting the ponderomotive force (re-written in terms of $\left.a=a_{0} e^{-r^{2} / w_{0}^{2}}\right)$ equal to the space-charge force gives

$$
\frac{-m c^{2}}{4 \gamma} \nabla a^{2}=\frac{e^{2}}{3 \epsilon_{0}} n_{e} r
$$


where $\gamma$ is the relativistic Lorentz factor. After differentiating the left hand side and assuming $a \approx \gamma$ (from the relation $\gamma^{2}=1+a^{2}[26]$, resulting in less than $10 \%$ error in the approximation for $a_{0} \geq 2$ )

$$
a_{0} e^{-r^{2} / w_{0}^{2}}=\frac{2}{3} k_{p}^{2} w_{0}^{2}
$$

Finally, setting the blowout radius $R=w_{0}$ the relation between the laser intensity, spot size, and electron density becomes

$$
k_{p}^{2} w_{0}^{2}=0.74 \sqrt{a_{0}}
$$

From simple scaling arguments Lu et. al. reported a coefficient of 1 on the right hand side of Eq. 2.19. The factor of two on the right hand side of Eq. 2.15 was determined through their simulations as the condition which provided the most stable self-guiding of the laser beam, which is necessary to drive the wake over $>1 \mathrm{~mm}$-scale distances (the importance of which will be seen in a later section).

In summary, plasma electrons are expelled from the focal region of a high intensity laser pulse by the ponderomotive force. After this short-duration pulse passes, electrons are pulled back toward the laser axis by the space-charge force of the stationary ions; in a frame moving with the laser pulse these electrons form a sheath around the ions. The electrons overshoot, setting up an electron plasma wave - the wake - which follows the laser pulse; this process is illustrated in Fig. 2.1. The first period of the wake is seen in the simulations of $\mathrm{Lu}$ et.al. to be nearly spherical for $2<a_{0}<4$, and the laser pulse is self-guided within the wake.

However, when the blown-out electrons return to the laser axis the electron density becomes very high just at the rear of the wake period, or "bucket". This manifests in a large local spike in the longitudinal electric field (since this phenomenon occurs on the laser axis where the radial field has only a longitudinal component) just at the back of the sphere, as seen in the simulations of Lu et.al.[1] and in Fig. 2.2. While this spike plays a critical role in the injection of background electrons into the first bucket, it is sufficiently localized so as not to cause the longitudinal electric field to deviate from linear. 


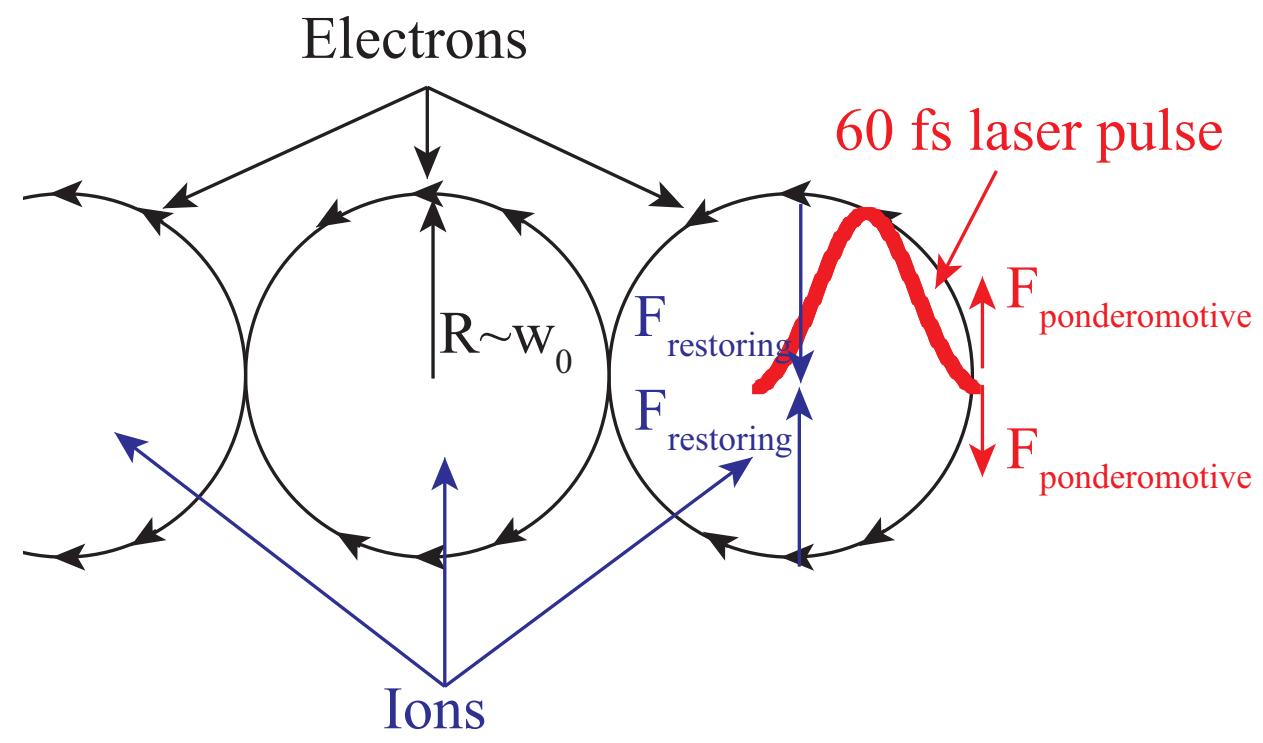

Figure 2.1: A focused, $a_{0}>2,60$ fs laser pulse (in red) is shown propagating to the right, where the frame is moving with the laser pulse. At the rising edge of the pulse the ponderomotive force "blows out" electrons (trajectories shown in black), while the stationary ions (not shown) provide a restoring space-charge force. Behind the laser pulse electrons are pulled back toward the laser axis and overshoot due to their inertia, and the resulting plasma wave wake has a radially directed electric field as described in the text. Along the laser propagation axis this field is purely longitudinal and is the accelerating force for LWFA. For a given plasma density and laser power, Eq. 2.15 determines the radius $\mathrm{R}$ of the blown-out region. The focusing geometry of the laser should be chosen such that the spot size $\mathrm{w}_{0} \approx \mathrm{R}$ in order to satisfy the matching condition and achieve stable laser guiding in the plasma. 


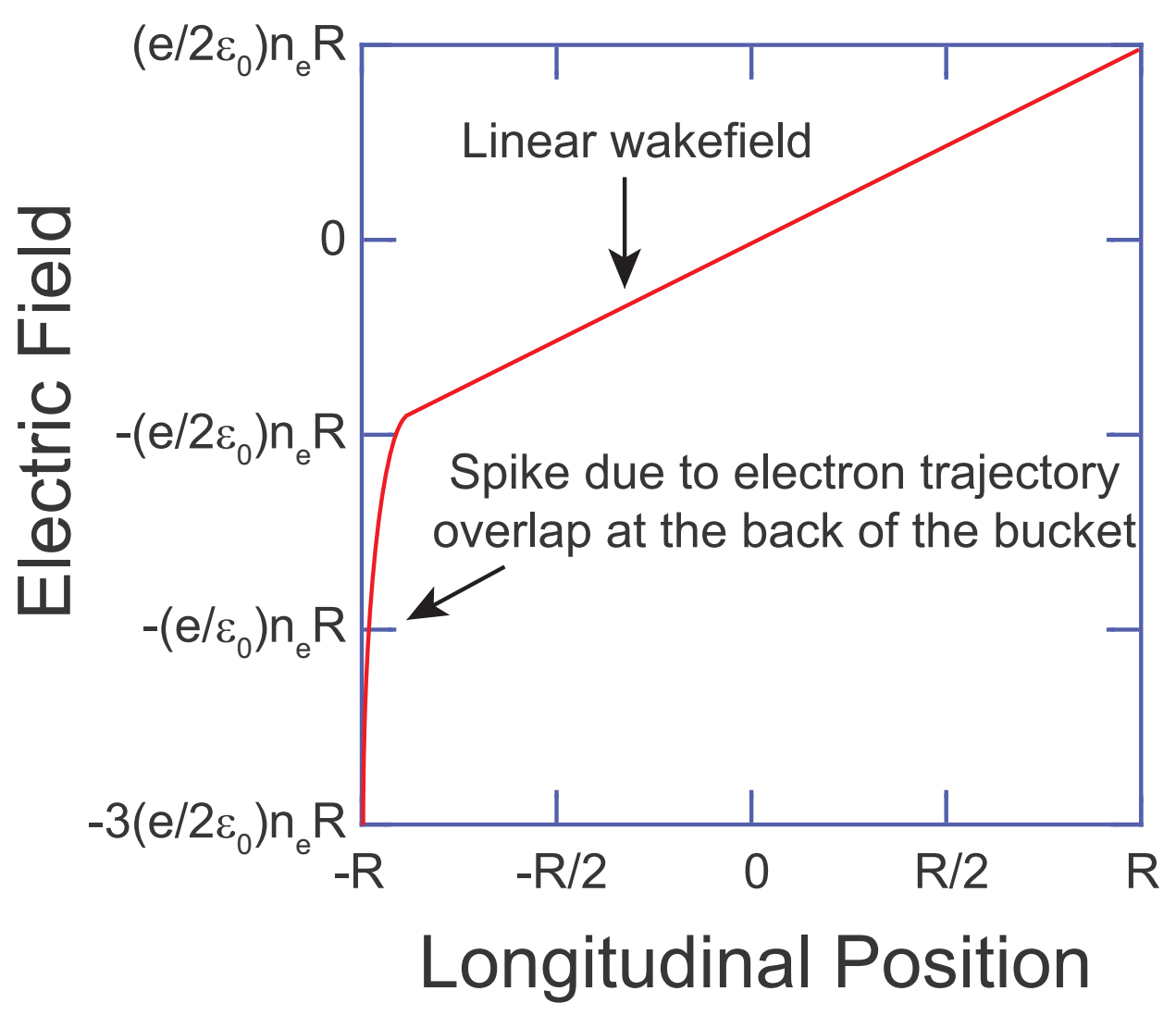

Figure 2.2: The longitudinal electric field profile (the field along the laser axis) of the first bucket is shown, where the localized spike at the back of the bucket is due to the locally high electron density arising from electron trajectory overlap at the laser axis. The width and depth of the spike are sensitive to the exact trajectories of the blown-out electrons, and in the simulations of Lu et. al.[1] the amplitude is roughly 3 times the linear maximum value. 
Additional observations from simulations indicate that, while the longitudinal electric field of the first bucket scales with density and blowout radius as expected from Gauss's law, the magnitude of the field is increased by a factor of 1.5. The resulting maximum electric field of

$$
E_{\max }=\frac{e}{2 \epsilon_{0}} n_{e} R
$$

observed in the simulations is attributed to the details of the trajectories of the blown-out electrons and hence the real shape of the bucket (including deviations from perfect spherical symmetry). This scaling has also shown very good agreement with experimental values for energy gain[14,13], and this value of the peak electric field is used throughout this thesis.

\subsection{Electron Injection and Trapping in the Wake}

\subsubsection{Self-injection}

An electron must find itself inside the wake structure with a velocity in the direction of laser propagation, or longitudinal velocity, equal to the wake phase velocity (derived in the next section, but corresponding to an electron kinetic energy of order $5 \mathrm{MeV}$ ) in order to be trapped and subsequently accelerated. Much of the current literature regarding LWFA, including results that will be presented in this thesis, employ the self-injection mechanism to trap blown-out electrons into the wake. Consider the motion of electrons as shown in Fig. 2.1: after being ionized and blown out at the front of the first bucket they move along a nearly circular arc around the ions before returning to the laser axis at the back of the bucket. This narrow region where trajectories overlap gives rise to the negative electric field spike in the longitudinal electric field profile at the back of the first bucket as shown in Fig. 2.2. As electrons move around the perimeter of the bucket they experience the radial electric field of the ions, where the component transverse to the laser propagation has already been discussed as the restoring force to the laser ponderomotive force. Additionally, the electrons feel the longitudinal component of the radial field, which is symmetric except for the spike at the back 


\section{Self-Trapped Electron Trajectory}

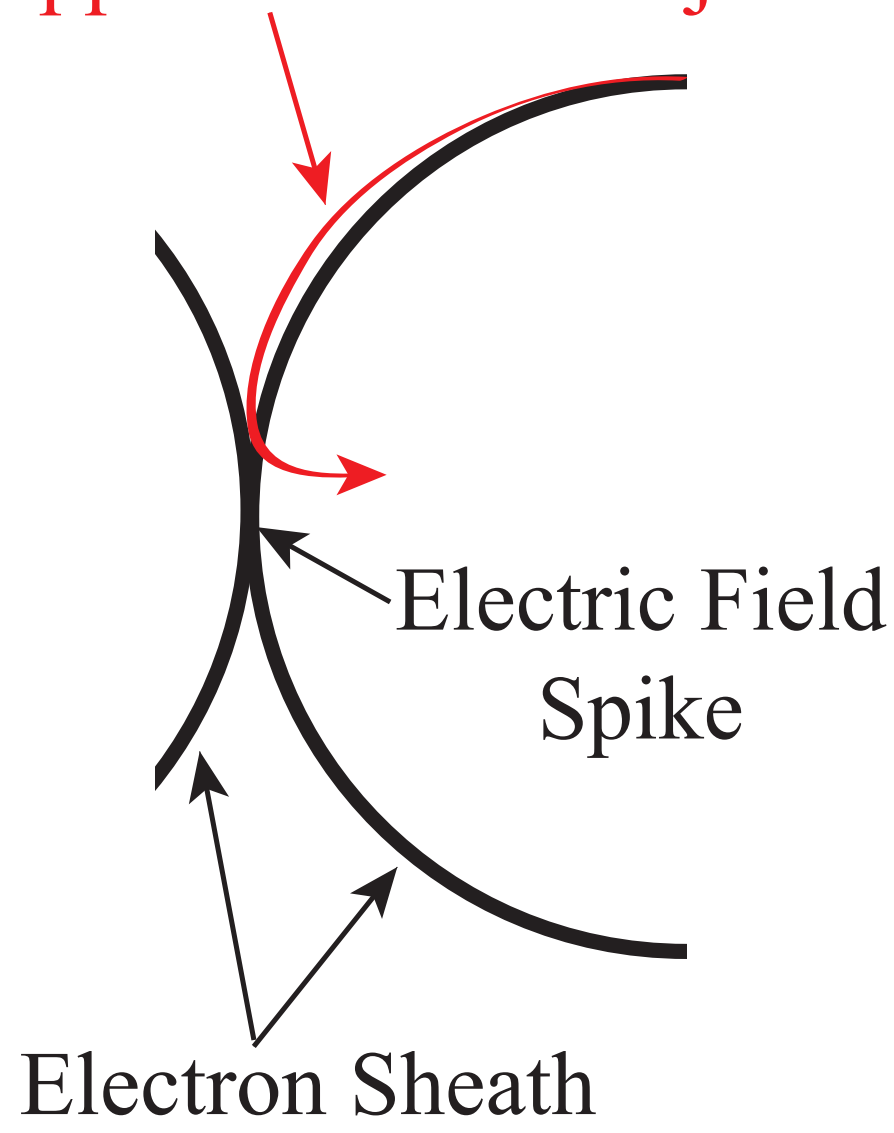

Figure 2.3: The electron sheath at the back of the first bucket and the front of the second bucket are shown (in black), along with the trajectory of a self-trapped electron (in red). The trapped electron's trajectory intersected the high electric field region at the back of the first bucket such that the field pushed the electron forward to a sufficient energy for it to travel at the wake phase velocity. 
of the bucket. This assymetry is necessary for electrons to achieve the required longitudinal velocity for trapping, as without it their longitudinal velocity would be the same as it was at the point of ionization (from the symmetry of the electric field profile).

In order for self-injection to occur some portion of the electrons must take a slightly different trajectory around the ions than that shown in Fig. 2.1. This modified trajectory is shown in Fig. 2.3, where the radius of the self-trapped electron trajectory is slightly larger than the nominal blowout radius. Rather than following the bulk of the electrons in the sheath, this electron crosses the sheath at the back of the first bucket and is pushed forward by the electric field spike. The electron becomes trapped only if it receives enough energy from the spike to reach the phase velocity of the wake.

The exact details of the shape of the electric field spike, and therefore the details of the self-trapping process and the necessary electron trajectories, are extremely sensitive to the exact laser and plasma profiles and are still under investigation. There have been several numerical studies of this trapping process[29, 30], which predict density thresholds for self-trapping of few- $10^{18}-10^{20} \mathrm{~cm}^{-3}$ for the blowout condition $2<\mathrm{a}_{0}<4$. This thesis will present data indicating that in He

plasmas this threshold occurs at $4 \times 10^{18} \mathrm{~cm}^{-3}$ for laser powers of $60 \mathrm{TW}$. However, it will also be shown that in order to achieve electron energy gains greater than 1 $\mathrm{GeV}$ the density must be reduced to less than $2 \times 10^{18} \mathrm{~cm}^{-3}$, requiring a different mechanism to trap electrons in the wake for high energy studies.

\subsubsection{Ionization-induced Injection}

A recently developed approach to injection at low densities is ionizationinduced injection $[15,16]$. The mechanism is enabled by adding trace amounts of a high-Z dopant gas (such as $\mathrm{N}_{2}$ or $\mathrm{O}_{2}$ ) to the He background. The dopant should be chosen such that its L-shell electrons will be ionized at a similar laser intensity to the K-shell electrons from the He gas, thereby allowing the dopant L-shell electrons to be blown-out and contribute to forming the wake. Conversely, the dopant Kshell electrons should have a sufficiently high ionization potential that they are 
not ionized (via ADK tunnel ionization[2]) until nearly the peak laser intensity, which is inside the wake as seen in Fig. 2.1. These electrons do not feel the strong transverse ponderomotive force of the laser, and are thus not blown-out with the rest of the electrons. Instead, the dopant K-shell electrons (which are born near the center of the bucket) slip backward in the wake near its axis. If they gain sufficient longitudinal momentum to be trapped before reaching the back of the bucket they can turn around and begin accelerating forward in the wake. This scheme has successfully allowed electron trapping at densities where self-trapping did not occur, i.e. below $4 \times 10^{18} \mathrm{~cm}^{-3}[17,13]$, and will be discussed in greater detail in the experimental results chapters where the technique was employed to increase the electron beam energy and reduce the energy spread.

\subsection{Energy Gain from LWFA}

Regardless of the mechanism responsible for trapping an electron in the wake, once it is injected into the wake region it will then accelerate under the $\sim \mathrm{GeV} / \mathrm{cm}$ longitudinal wakefield. Since the laser is propagating in a plasma its group velocity is reduced from the vacuum value of $\mathrm{c}$, and electrons accelerating in the wake begin to outrun it. Additionally, as the laser gives energy to the wake the front of the pulse is pump depleted, which effectively slows the wake further. This section will calculate the length in the laboratory over which an electron remains in the accelerating portion of the wake, known as the "dephasing length," as well as the resulting energy gain.

\subsubsection{Dephasing and Pump Depletion}

From Maxwell's equations and the definition of the current density $\mathbf{J}=q n \mathbf{v}$ the well-known dispersion relation for electromagnetic waves (light) in a plasma is given by

$$
\omega^{2}=\omega_{p}^{2}+k^{2} c^{2}
$$




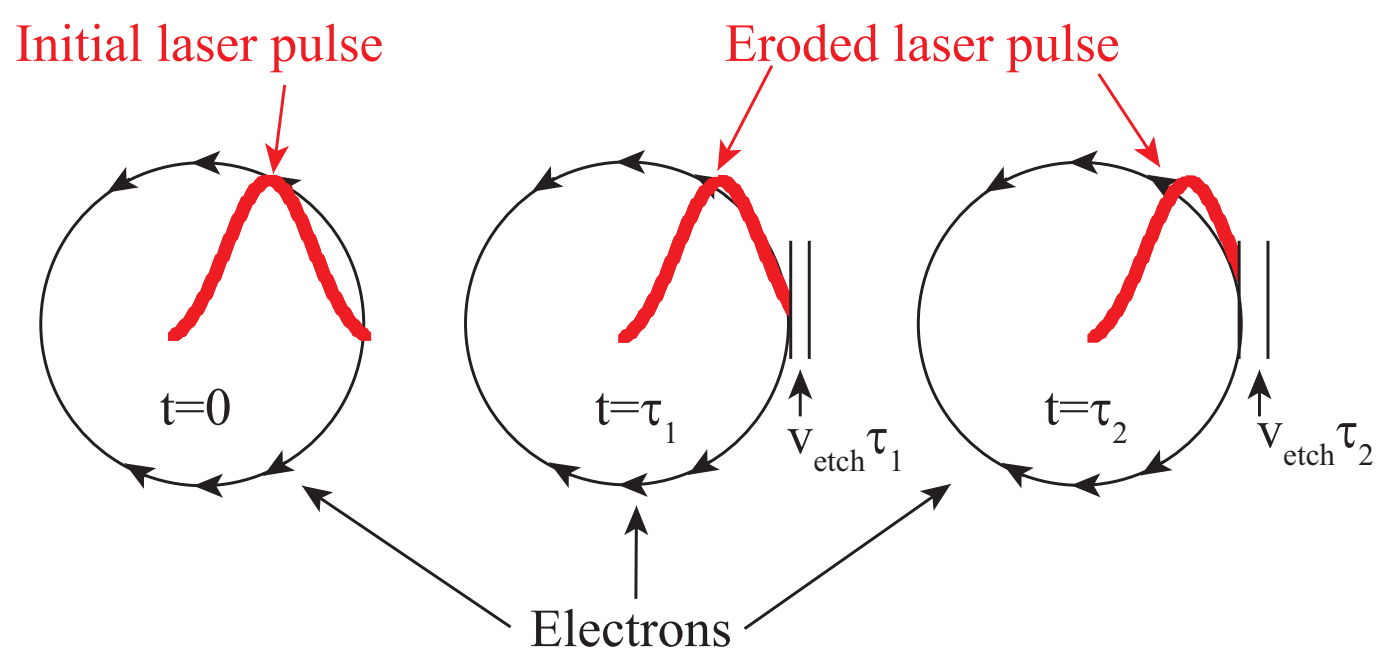

Figure 2.4: As the laser propagates it continuously loses energy to the wake via red-shifting of laser photons. As photons red-shift they move more slowly in the underdense plasma, and the head of the laser pulse erodes backward. The left panel shows the initial laser pulse before any pump depletion, while the center and right panels show increasing amounts of pump depletion corresponding to increased laser propagation distances in the plasma. The front of the laser pulse moves backward in the plasma at the etching rate $v_{\text {etch }}=c n_{e} / n_{c}$.

with $\omega(\mathrm{k})$ the frequency (wavevector) of the light and $\omega_{p}$ the electron plasma frequency. The group velocity $v_{g}=\frac{\partial \omega}{\partial k}$ for propagation through underdense plasma is then

$$
v_{g}=c\left(1-\frac{\omega_{p}^{2}}{\omega_{0}^{2}}\right)^{1 / 2} \simeq c\left(1-\frac{n_{e}}{2 n_{c}}\right)
$$

where it is assumed that $n_{e}<<n_{c}$ so that $k \approx k_{0}$ and $k_{0} c=\omega_{0}$. A central laser wavelength of $800 \mathrm{~nm}$ corresponds to a critical density $n_{c}=1.75 \times 10^{18} \mathrm{~cm}^{-3}$, so for a plasma density $n_{e}=3 \times 10^{18} \mathrm{~cm}^{-3}$ the laser group velocity $v_{g}=0.999 \mathrm{c}$.

As the laser propagates and continuously blows out electrons, energy is continuously being given to the wake. Since the number of photons must be conserved, this energy lost to the wake comes in the form of photon deceleration, or red-shifting of the photons. These longer wavelength photons move more slowly in the plasma, and consequently the head of the laser pulse erodes backward. This process, called pump depletion, is illustrated schematically in Fig. 2.4. This theory has been developed by Decker et.al[31] in 1-D, and predicts an etching rate 
$v_{\text {etch }}=c n_{e} / n_{c}$ for the head of the laser pulse moving backward in the wake. The 2-D simulations of Decker et.al. and the 3-D simulations of Lu et.al.[1] are in very good agreement with the non-linear rate calculated in 1-D. The etching length, or pump depletion length $L_{p d}$, for a pulse of duration $\tau$ is then given by

$$
L_{p d}=\frac{c}{v_{\text {etch }}} c \tau=\frac{n_{c}}{n_{e}} c \tau
$$

Since the head of the laser pulse is eroding due to pump depletion, the source of electron blowout is also slipping backward in the pulse. Relative to a trapped electron, then, the bucket is moving backward at $v_{\text {etch }}$, which acts to reduce the wake phase velocity from the laser group velocity. The wake phase velocity $v_{\phi}$ is then given by

$$
v_{\phi}=v_{g}-v_{e t c h}=c\left(1-\frac{3 n_{e}}{2 n_{c}}\right)
$$

An electron is trapped in the wake if it obtains a kinetic energy such that its longitudinal velocity is equal to the phase velocity of the wake. For the $n_{e}=3 \times 10^{18}$ $\mathrm{cm}^{-3}$ example above, where $v_{\phi}=0.997 c$ (reduced from the $0.999 \mathrm{c}$ group velocity of the laser), this corresponds to a kinetic energy $K E=(\gamma-1) m c^{2}=6.6 \mathrm{MeV}$.

As the electron accelerates in the wake it continues to gain velocity (although most of the energy is going to the mass increase), and begins to outrun the wake. If the electron is assumed to be trapped at the rear of the bucket as shown in Fig. 2.5 (near the position -R in Fig. 2.2), the furthest it can move forward while remaining in the accelerating portion of the wake is the bucket radius $\mathrm{R}$; after this point (where the electron passes the center of the bucket and hence the zero-crossing of the electric field) the electric field is decelerating for the electron and it begins to lose energy. If it is further assumed that the electron is traveling at $\mathrm{c}$, it will remain in this accelerating phase for a laboratory distance termed the dephasing length $L_{\text {deph }}$ which is calculated to be

$$
L_{\text {deph }}=\frac{c}{c-v_{\phi}} R=\frac{2}{3} \frac{n_{c}}{n_{e}} R
$$

An electron that remains in the wake for a distance greater than the dephasing length will begin to see a decelerating electric field, and hence lose energy. Con- 


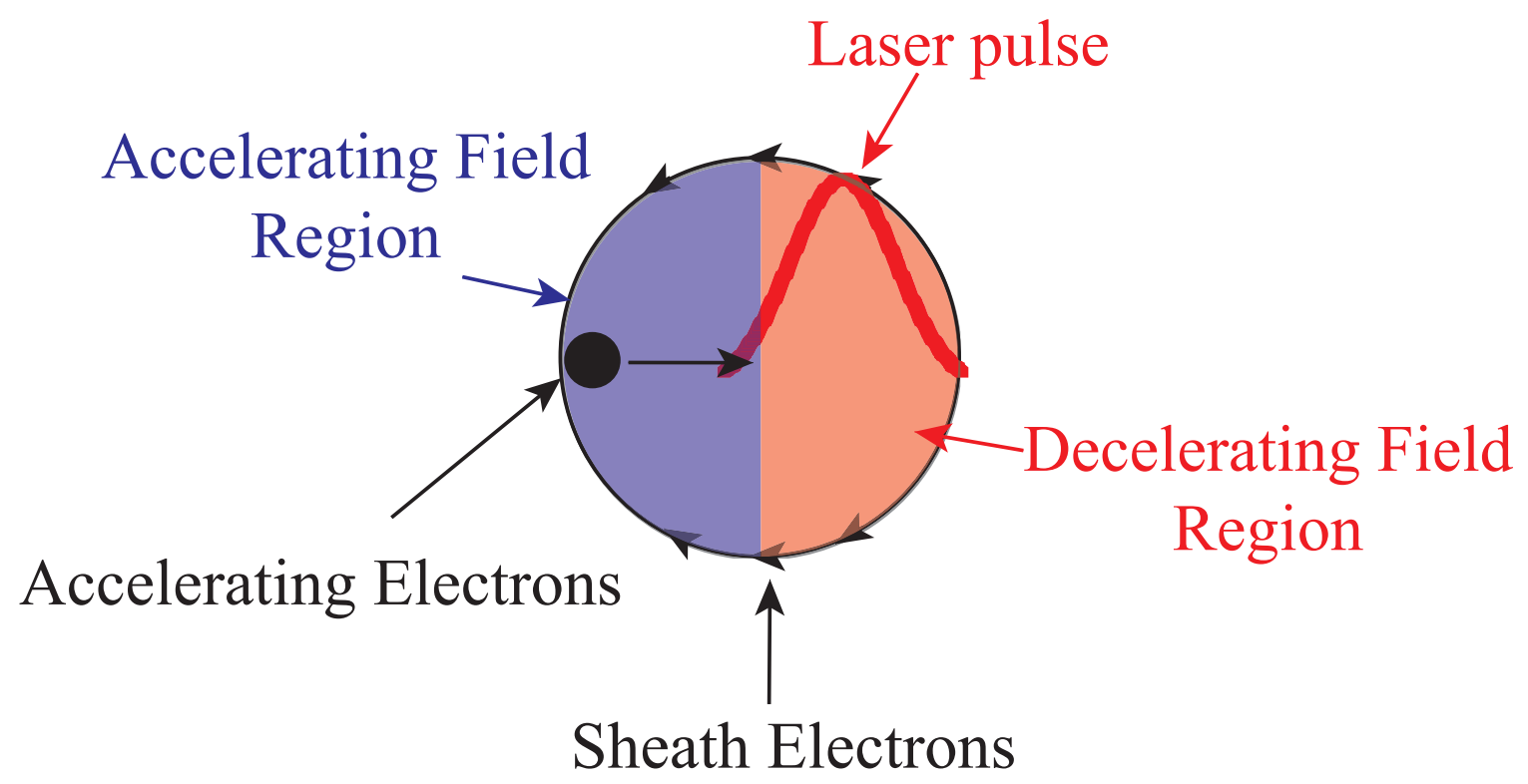

Figure 2.5: Electrons injected at the back of the laser pulse are in a region of accelerating field. Due to the spherical symmetry of the ions in the wake, once the electrons have moved past the center of the wake the sign of the electric field reverses as shown in Fig. 2.2. Electrons that propagate through this portion of the wake lose energy in the decelerating electric field. Electrons traverse the accelerating field region over a laboratory distance of one dephasing length. 
versely, if the length of the plasma is shorter than $L_{\text {deph }}$ electrons will not reach the zero-crossing of the electric field, and will therefore not achieve the maximum possible energy gain. This sets an experimental requirement that the length of the plasma be set as close to the dephasing length as possible in order to maximize the overall energy gain of the electron beam. There is also an additional requirement that the length of the plasma be kept below the pump depletion length, as this is the greatest distance over which a wake can be stably driven.

\subsubsection{Dephasing-limited energy gain}

The dephasing limited energy gain $W_{\max }$ of an electron in the blowout regime of $L W F A$ is

$$
W_{\text {max }}=\int \mathbf{F} \cdot \mathbf{d} \mathbf{l}=e E_{\text {ave }} L_{\text {deph }}
$$

where $\mathbf{F}=-e \mathbf{E}, \mathbf{E} \cdot \mathbf{d} \mathbf{l}=-E_{\text {ave }} L_{\text {deph }}$ for a linear electric field over the dephasing length, $E_{\text {ave }}=E_{\max } / 2$ from Eq. 2.20, and it is assumed that $L_{p d} \geq L_{d e p h}$. Substituting Eq. 2.20 and Eq. 2.25 into Eq. 2.26 with the definitions for the blowout radius (Eq. 2.15) and the laser normalized vector potential (Eq. 2.16) and re-arranging, the energy gain expressed in terms of laser power and electron density is

$$
W_{\text {max }}(G e V)=1.7\left(\frac{P}{100 T W}\right)^{1 / 3}\left(\frac{10^{18} \mathrm{~cm}^{-3}}{n_{e}}\right)^{2 / 3}
$$

for $800 \mathrm{~nm}$ laser light. Note that the energy gain depends inversely on the electron density; even though the wake electric field is reduced as the electron density is reduced, the dephasing length increases faster. The lower limit for electron densities that can produce high energy electron beams is set by the requirement for self-guiding, i.e. $P>P_{\text {crit }}$. In order to achieve electron energy gains exceeding 1 GeV with presently available laser systems $\left(\mathrm{P} \leq 100 \mathrm{TW}\right.$, where $P_{\text {crit }}=100 \mathrm{TW}$ corresponds to an electron density of $3 \times 10^{17} \mathrm{~cm}^{-3}$ ), electron densities below $\sim 2.2 \times 10^{18}$ $\mathrm{cm}^{-3}$ are required and the laser must be self-guided over a dephasing length of at least $7.5 \mathrm{~mm}$. As discussed in the injection section above, operating at these densities precludes self-trapping in favor of ionization-induced injection. Later chapters 
will discuss experiments that have demonstrated electron energies above $1 \mathrm{GeV}$ using this injection mechanism, but in a continuous energy spread beam, and a technique which has demonstrated a $\sim 0.5 \mathrm{GeV}$ beam with a significant reduction in energy spread by terminating the ionization-induced injection process. 


\section{Chapter 3}

\section{Experimental Setup}

This chapter presents the experimental setup for the Laser Wakefield Acceleration studies performed with the Callisto laser system. The gas cell and gas jet targets used for the experiments are described, as well as the diagnostic suite which was developed to characterize the laser, plasma, and electron beam parameters.

\subsection{Callisto Laser System}

The Callisto Laser System is located at the Lawrence Livermore National Laboratory and is part of the Jupiter Laser Facility. It is a Ti:Sapphire chirpedpulse amplification (CPA)[32] system with a central wavelength of $800 \mathrm{~nm}$ which is capable of producing $200 \mathrm{TW}, 60$ fs laser pulses at a rate of 1-2/hour; a block diagram of the key components of the system is shown in Fig. 3.1. CPA laser systems "chirp" (or stretch in time) initially short (and therefore broadband) laser pulses to reduce the optical intensity during subsequent amplification stages of the system, as shown in Fig. 3.2. After each amplifier section the beam diameter is increased, so that after the final amplification the beam diameter is large enough to safely re-compress the beam temporally to short duration. The temporal stretching and compressing of the beam are achieved with diffraction gratings, where the first order reflection when stretching separates the various wavelengths of the broadband laser beam and recombines them when compressing.

The beam originates with a Femtosource oscillator (from Femto Lasers) 


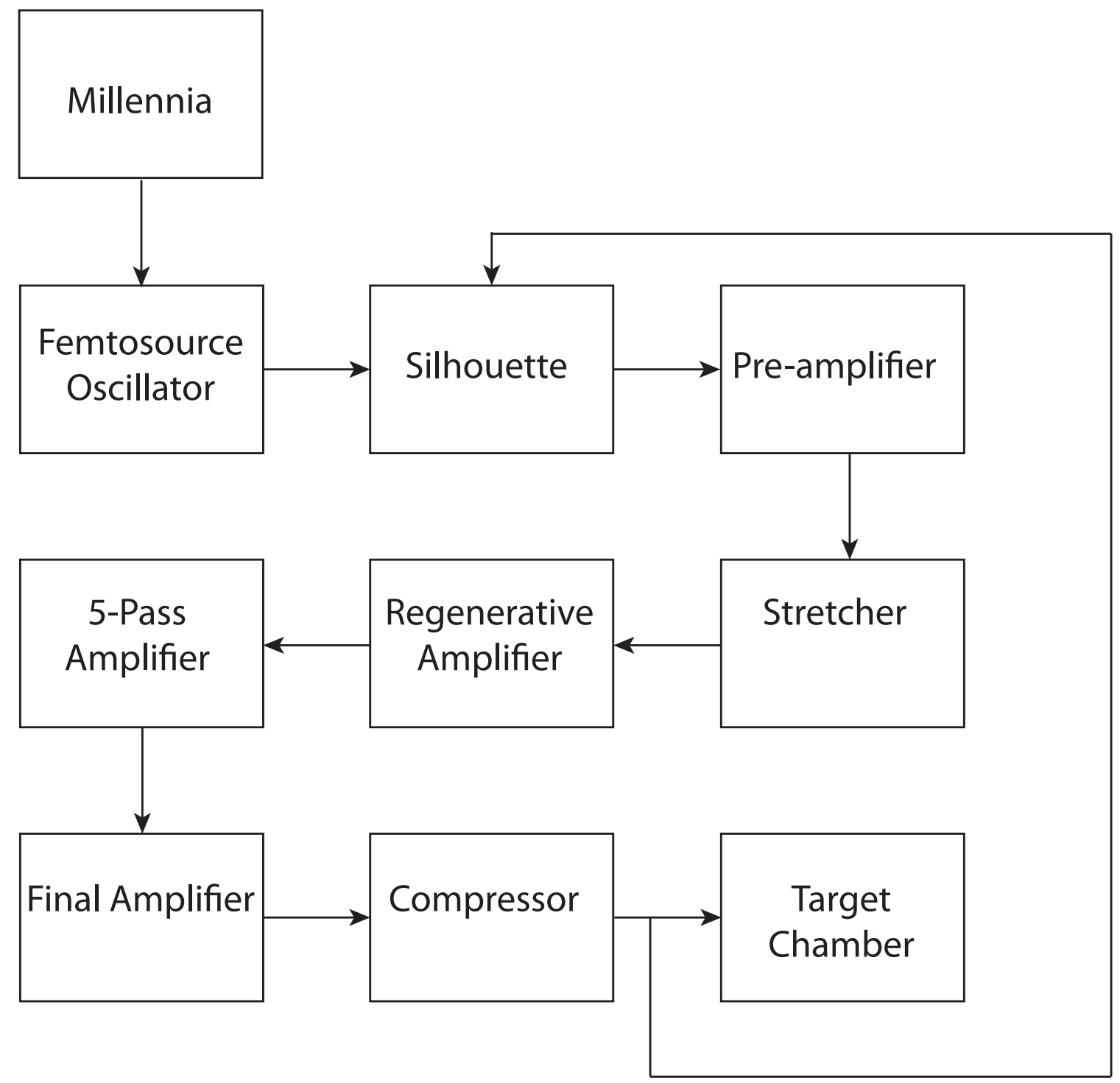

Figure 3.1: Block diagram of the main components of the Callisto Laser System. 


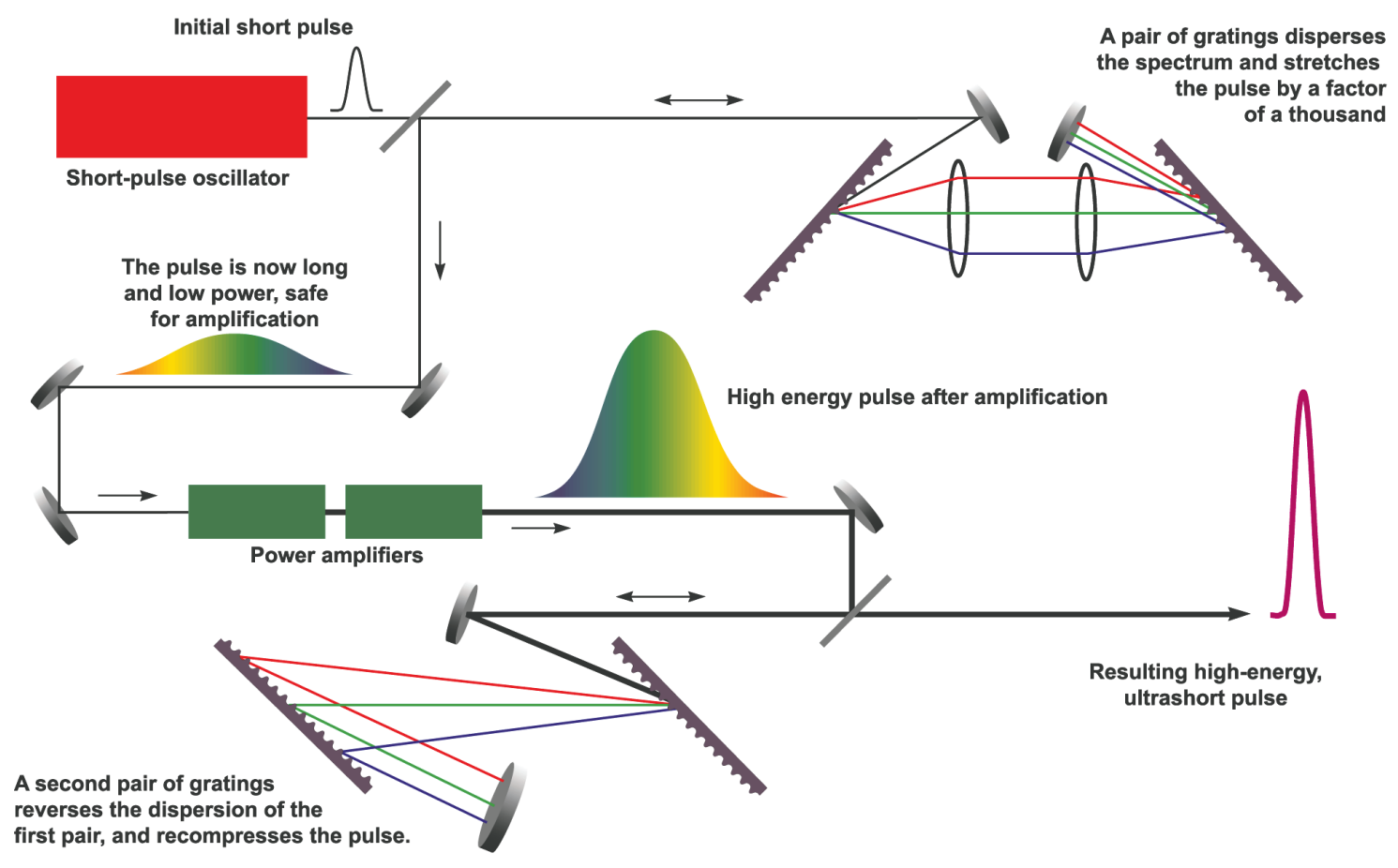

Figure 3.2: Typical stages of a CPA laser system.

which is pumped by a diode-driven Millennia CW laser (from Newport). The Millennia operates at $532 \mathrm{~nm}$ and $3.8 \mathrm{~W}$. The oscillator produces a train of broadband pulses centered at $800 \mathrm{~nm}$ wavelength and separated by $12.2 \mathrm{~ns}(82 \mathrm{MHz})$. Ten pulses per second are selected from this pulse train by a pulse-picker Pockels cell, giving rise to the $10 \mathrm{~Hz}$ alignment mode of operation available at Callisto. A Silhouette (from Coherent) spatial light modulator is used to minimize phase distortions across the laser spatial profile and improve the beam quality and final compression; the spectrum of the compressed beam is measured with an Ocean Optics fiber spectrometer and feedback from that measurement is sent to the Silhouette to determine the required modulations to the pulse. The bandwidth of each pulse is $36 \mathrm{~nm}$ entering the stretcher. Prior to being stretched the beam is sent through a pre-amplifier section to reduce the eventual number of passes required in the regenerative amplifier; each pass through the regenerative amplifier accumulates amplified spontaneous emission (ASE) which is not compressible and becomes a pre-pulse (light arriving at the target ahead of the main pulse). The 
contrast of the laser is defined as the peak power (or intensity if focused) of the main pulse divided by the peak power of the pre-pulse and is measured with a water cell during a high power laser shot to be of order $10^{6}$. The energy in each pulse entering the regenerative amplifier is $\sim 1 \mu \mathrm{J}$; after 8 round trips through the cavity the energy is increased to $4 \mathrm{~mJ}$. The 5-pass amplifier then increases the energy to $250 \mathrm{~mJ}$. The Ti:Sapphire crystal in the regenerative (5-pass) amplifier is pumped by a Quanta-Ray GCR-4 diode laser delivering $100 \mathrm{~mJ}$ (650 mJ) of $532 \mathrm{~nm}$ laser light light to the crystal. The final amplifier section consists of two additional crystals which are pumped by $14 \mathrm{~J}$ and $86 \mathrm{~J}$ of frequency-doubled Nd:YAG laser light (with a wavelength of $527 \mathrm{~nm}$ ) produced by the Janus Laser System also located at the Jupiter Facility. The limit on the rate for high power shots is heating of this final amplifier section, which increases the final energy of the Callisto laser beam (in single shot mode) to up to $30 \mathrm{~J}$. The pulse is then re-compressed using diffraction gratings (see Fig. 3.2) to reverse the chirp introduced by the stretcher; the compressed pulse has a bandwidth of 20-25 nm and a duration of 55-65 fs FWHM. After re-compressing the pulse to short duration it is transported to the target chamber, where it is focused by an $\mathrm{f} / 8$ off-axis parabola to a $1 / \mathrm{e}^{2}$ spot size $\mathrm{w}_{0}=15 \mu \mathrm{m}$ containing $30-50 \%$ of the laser energy.

\subsection{General Experimental Setup of the Callisto Target Chamber}

The general experimental setup used for the LWFA experiments described in this thesis is shown in Fig. 3.3. The main pulse from the compressor is reflected onto a 6" diameter off-axis parabola (OAP) with a 1-m focal length. The beam diameter at the OAP is 5.25 ", resulting in an $\mathrm{f} / 8$ focusing geometry. The focal spot has a $1 / \mathrm{e}^{2}$ radius $\mathrm{w}_{0}=15 \mu \mathrm{m}$ containing $30-50 \%$ of the laser energy. The gas target (introduced in the next section) is placed at the focus of the laser. After an introduction to the targets used in these experiments, the remainder of this chapter describes the diagnostics employed to characterize the high energy electrons produced from the LWFA mechanism, the laser light transmitted through 


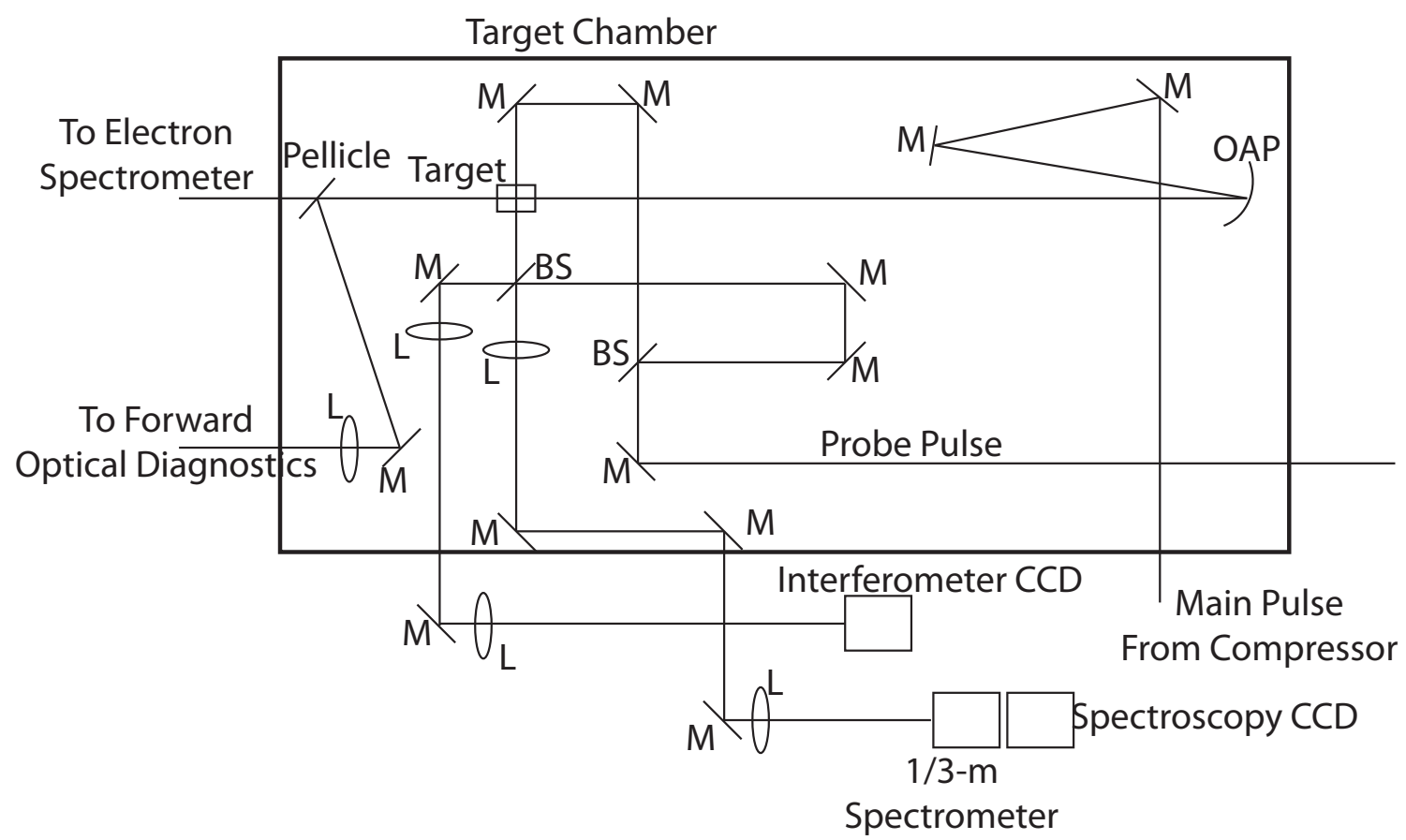

Figure 3.3: The otical layout inside the Callisto target chamber. The main laser pulse and the interferometer probe pulse are shown, as well as the interferometry and spectroscopy optical layouts. The probe pulse is split from the main pulse at the output of the 5-pass amplifier and compressed to $100 \mathrm{fs}$ duration with a secondary compressor. After traversing the target the laser pulse is partially reflected by a $5 \mu \mathrm{m}$ pellicle, while the remaining laser light is dumped on a $100 \mu \mathrm{m}$ Al light shield at the target chamber wall. Electrons pass through the pellicle and the light shield to enter the dipole magnet outside the vacuum chamber. The reflected laser light is collimated before leaving the chamber and is directed to optical diagnostics that measure the spot size and spectrum of the beam after traversing the plasma. 
the plasma, and the electron density and gas composition along the target.

\subsection{Targets}

\subsubsection{Gas Jets}

For the majority of the experiments performed at electron densities above $4 \times 10^{18} \mathrm{~cm}^{-3}$, a supersonic jet of He gas served as the target. The diameter of the jet determined the length of the plasma which could be produced, and from the dephasing length equation (Eq. 2.25) this set an optimum electron density and maximum energy gain for that jet diameter. In order to increase the maximum electron beam energy for a given laser power, Eq. 2.26 indicates that the electron density must be reduced and the length of the plasma increased (corresponding to a longer dephasing length at lower density). For this reason three gas jets of different diameters were used during the experiments in order to change the length of the plasma.

\subsubsection{Gas Cells}

For densities below $4 \times 10^{18} \mathrm{~cm}^{-3}$, a variable length gas cell replaced the gas jet. This is primarily because at these densities plasma lengths of $\sim 1 \mathrm{~cm}$ are required, and uniform gas profiles could not be achieved from our gas jets at these densities and lengths. The gas cell also allowed for variation of the gas composition along the target via independent filling of two stages separated by an aperture to allow the laser to pass between the stages. A schematic of the gas cell and a more detailed description of its key features are given in Chs.??.

\subsection{Diagnostics}

A suite of diagnostics allows the characterization of the plasma density, the laser propagation through the plasma, and the resulting electron beams. Each diagnostic that was used throughout the study is introduced here, while subsequent 
chapters will state which diagnostics each set of experiments employed and in what configurations.

\subsubsection{Electron Spectrometer}

In order to unambiguously determine the output electron beam energy and deflection angle at the plasma exit, we have implemented a two-screen electron spectrometer. This system is shown in Fig. 3.4 and is comprised of a dipole magnet followed by two Fujifilm image plates. By measuring the electron beam position on each plate, both the energy and deflection angle at the plasma exit are determined through the relativistic equations of motion.

The dipole magnet is comprised of two permanent magnetic plates, each measuring $20.3 \mathrm{~cm}$ long by $7.6 \mathrm{~cm}$ high. The field between the plates is determined by their separation distance, with $\mathrm{B}=0.46 \mathrm{~T}$ for a plate separation of $3.5 \mathrm{~cm}$. The magnet entrance is located $62.5 \mathrm{~cm}$ from the plasma exit. In order to resolve the electron energies, two Fujifilm BAS-MS image plates are placed after the exit of the magnet at distances of $144.6 \mathrm{~cm}$ and $203.8 \mathrm{~cm}$ from the plasma exit. They are read with a Fujifilm FLA-7000 scanner providing 200 micron spatial resolution. There is a minimum 20 minute delay between image plate exposure and scanning to minimize error introduced by fading effects in the plates [33].

In order to uniquely solve for the electron beam energy and initial deflection angle consider the relativistic Lorentz equation

$$
\gamma m \frac{d \mathbf{v}}{d t}=q \mathbf{v} \times \mathbf{B}
$$

where $\gamma$ is the electron beam energy normalized to $511 \mathrm{keV}, \mathrm{m}$ (q) is the electron rest mass (charge), and $\mathrm{B}$ is the field strength of the dipole magnet. While the electrons traverse the magnetic field they undergo Larmour motion at the relativistic gyrofrequency, $\omega_{c}=q B / \gamma m$, which is determined by the beam energy for a given field strength. Allowing for an initial deflection angle $\theta$ of the electron beam from the laser axis and solving Equation 3.1 for the electron orbit due to the field yields the vertical position $\left(\mathrm{y}_{\text {exit }}\right)$ of the electrons at the magnet exit 


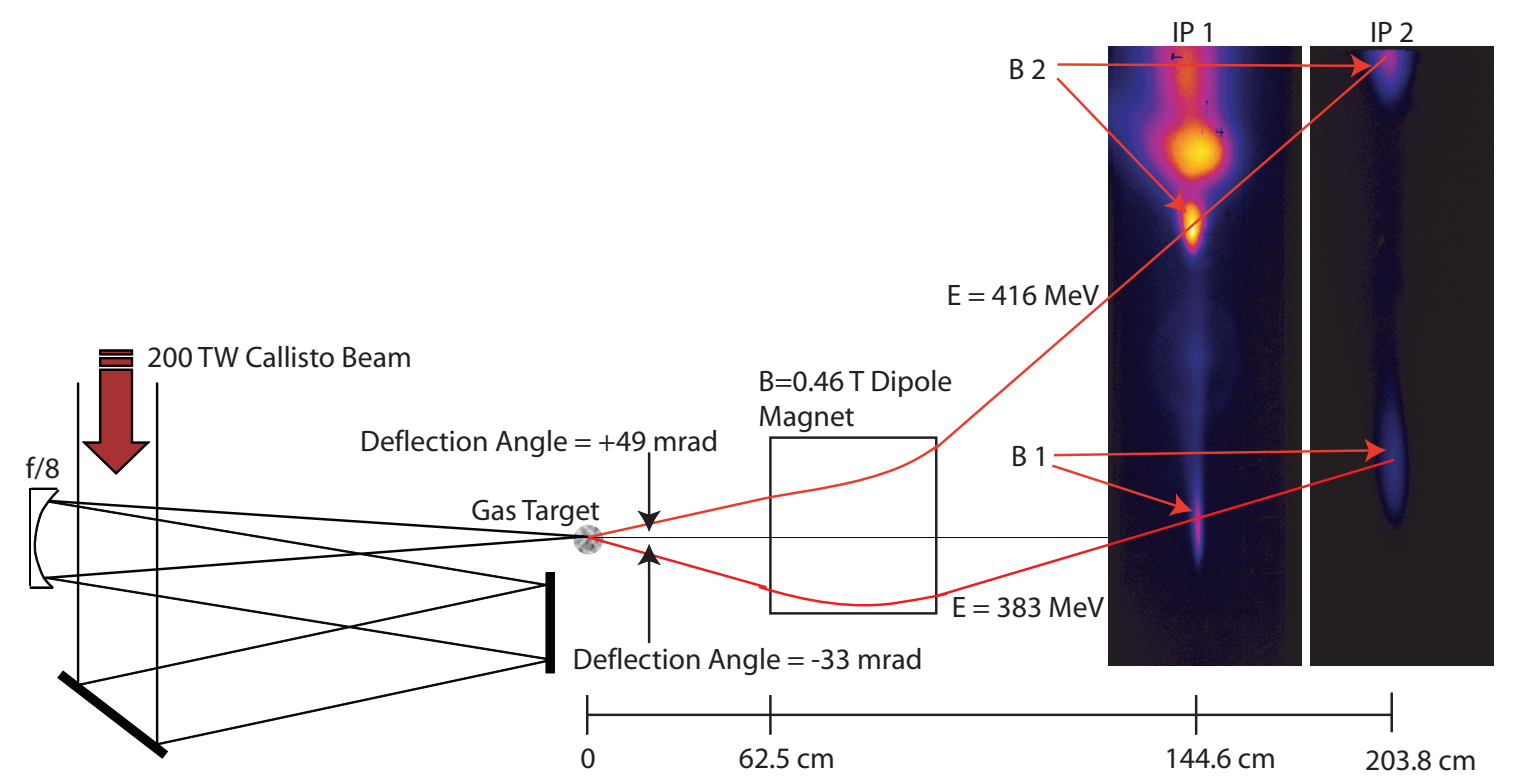

Figure 3.4: The 200 TW Callisto laser is focused by an f/8 off-axis parabola to a vacuum spot size of $\mathrm{w}_{0}=15$ microns at the leading edge of a gas target. The target delivers He gas at neutral densities of $1 \times 10^{18}-1 \times 10^{19} \mathrm{~cm}^{-3}$. A dipole magnet is placed after the target, followed by two image plates. The displayed trajectories through the magnet correspond to the features labeled B1 and B2 on the image plates.

$$
y_{\text {exit }}=z_{0} \tan \theta+\frac{v}{\omega_{c}}(\cos \theta-\cos \phi)
$$

where $z_{0}$ is the distance from the plasma exit to the magnet entrance and $\phi$ is the electron beam angle relative to the laser axis at the magnet exit.

Since the vertical displacement of the electron beam is known in two positions after the magnet we obtain a second relation for $\mathrm{y}_{\text {exit }}$

$$
y_{\text {exit }}=y_{1}-d_{1} \tan \phi
$$

where $\mathrm{L}$ is the length of the magnet, $\mathrm{d}_{1}$ is the distance from the magnet exit to the front image plate, $\mathrm{y}_{1}$ is the height of the electron beam on the front image plate, and $\tan \phi$ is the slope of the electron beam trajectory as determined from both image plates. The system of equations is closed by relating the beam energy to the deflection angle by $\mathrm{L}=\mathrm{r}_{L}(\sin \phi-\sin \theta)$, with the Larmour radius $\mathrm{r}_{L}=\mathrm{v} / \omega_{c}$. 
Figure 3.4 shows the result of a 145 TW laser pulse incident on a plasma with an electron density of $3.2 \times 10^{18} \mathrm{~cm}^{-3}$. In this particular example there is more than one bunch of accelerated electrons visible on the image plates. For the bunch labeled B1 on each image plate, the deviation from the laser axis is $3 \mathrm{~mm}$ for the first plate and $26 \mathrm{~mm}$ for the second plate.

If the electron beam is assumed to have no deflection angle at the plasma exit, using only the first image plate position to solve the equations of motion in the magnet results in an energy measurement of $5 \mathrm{GeV}$. Using only the second image plate with the same assumption gives an electron beam energy of $1.4 \mathrm{GeV}$. However, since the beam height is known in two locations the trajectory of the beam back to the exit plane of the magnet is determined. It is now not possible to solve the equation of motion without allowing for an initial deflection angle at the plasma exit. For the data in Figure 3.4, the electrons exit the plasma with an angle of $-33 \pm 1.5 \mathrm{mrad}$; this corresponds to a beam energy of only $383 \pm 20 \mathrm{MeV}$.

The same analysis can be applied to each successive electron beam. For the bunch labeled B2, the beam height is 11.7 (18.55) $\mathrm{cm}$ on the first (second) plate, which independently corresponds to 165 (195) MeV assuming no deflection. Solving using both measurements yields an energy of $416 \pm 30 \mathrm{MeV}$ with a deflection angle of $+49 \pm 3 \mathrm{mrad}$. This indicates that not only is it necessary to allow for initial deflections, but that each electron beam trajectory must be analyzed independently as the deflection angle can vary between features from the same experiment.

Figure 3.5 shows the results from analyzing $>60$ of our recorded electron beams. Each beam has been analyzed first using both image plates, and then again with only the front plate. The solid line in Figure 3.5a corresponds to equal energy measurements for both methods. Points above this line correspond to electron beams that underwent a downward deflection at the plasma exit, while points below the line correspond to beams that had a positive deflection angle. Every beam suffered from some initial deflection, as shown in Figure 3.5b.

For the lowest energy electron beams the energy measured with the twoscreen system closely follows the solid line of Figure 3.5a. This is to be expected, since lower energy beams are deflected more by the dipole magnet. As the electron 
beam energy increases, the magnet deflection decreases, and the effect of the initial deflection becomes more pronounced. This necessitates a two-screen electron spectrometer system in order to resolve $\mathrm{GeV}$-scale electron beams.

\subsubsection{Interferometry}

A portion of the main Callisto beam that is transmitted through a "leaky" mirror in the 5-pass amplifier is independently compressed to $100 \mathrm{fs}$ and is available as a probe beam. The experiments described in this thesis employ the probe for interferometry in a Mach-Zehnder configuration; aligning the signal arm of the probe to intersect the target perpendicular to the main beam path allows the electron density along the plasma to be measured. The probe is timed to arrive 20 ps after the main pulse exits the plasma, before ion motion can occur. The spatial resolution is $125 \mu \mathrm{m}$, and the field of view is $11.8 \mathrm{~mm}$. Probe light is 1:1 relay imaged from the target plane to a 16-bit CCD camera which records the interferograms. The temporal resolution is determined by the duration of the probe, which is 100 fs. The optical path for the entire target chamber is shown in Fig. 3.3.

The 10-20 $\mu \mathrm{m}$ size of the wake is not resolvable by our imaging system. The density that is measured is the background density of the bulk plasma produced not only by the high intensity focal region of the laser but also by the wings of the laser pulse (i.e. wherever the intensity is above the He ionization threshold). This region is typically for our plasmas $1-2 \mathrm{~mm}$ in diameter. The cold ions in this region move on an acoustic time-scale; even a $100 \mathrm{eV}$ He ion can only move $1 \mu \mathrm{m}$ in 20 ps (compared to the mm-scale bulk plasma size). Additionally, 20 ps corresponds to $6 \mathrm{~mm}$ of propagation at c. When examining the interferogram of a 5-10 $\mathrm{mm}$ long plasma there are no discernable differences in the plasma density from one end to the other, further indicating that the ion motion is slow enough to be neglected on this time scale. The background electron density is then calculated by Abel inverting[34] the interferograms of this bulk plasma, and when compared with the energy gain achieved in various length plasmas provides very good agreement with calculations using the equations from the previous chapter. 

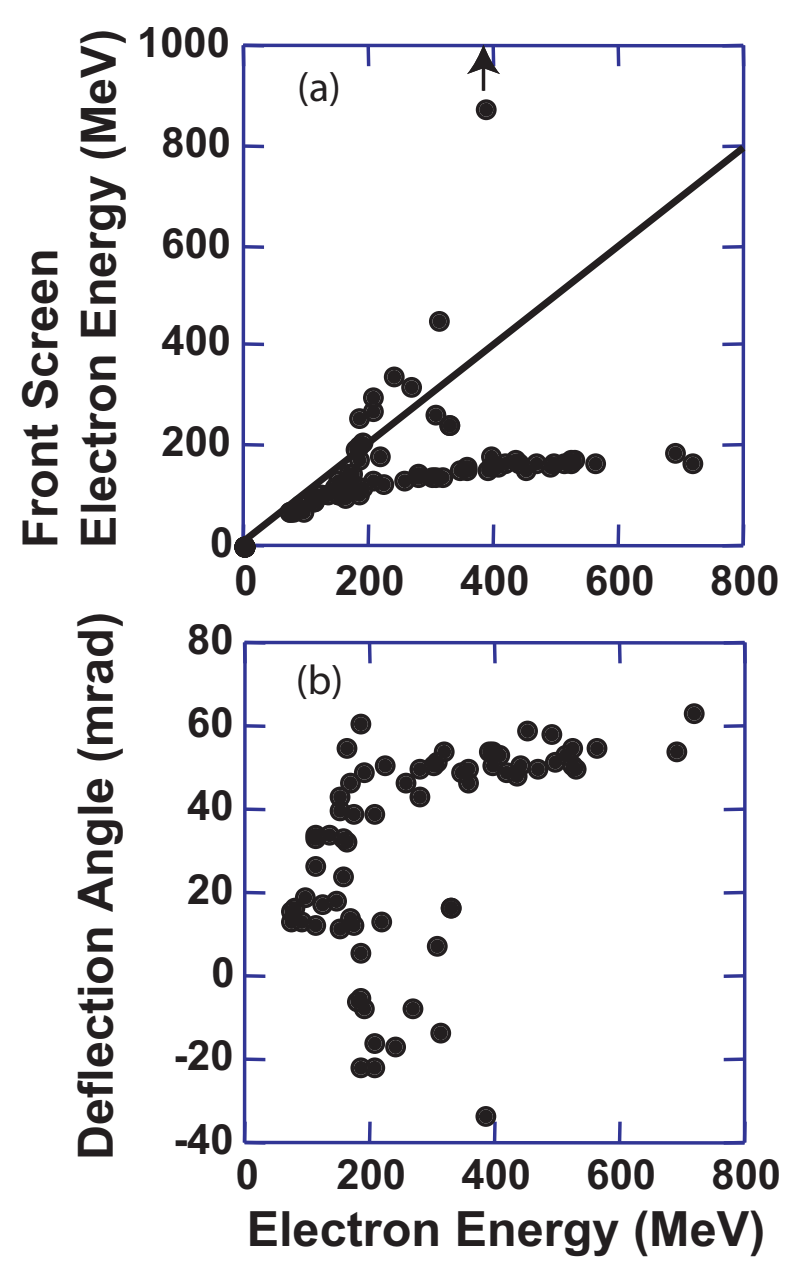

Figure 3.5: a) The energy of each of our electron beams is measured first using both image plates and then again with just the front image plate. The solid line divides electron beams with positive (below the line) and negative (above the line) deflection angles. b) The deflection angles for all electron beams are shown; there is no apparent correlation to beam energy. 


\section{Laser Propagation}

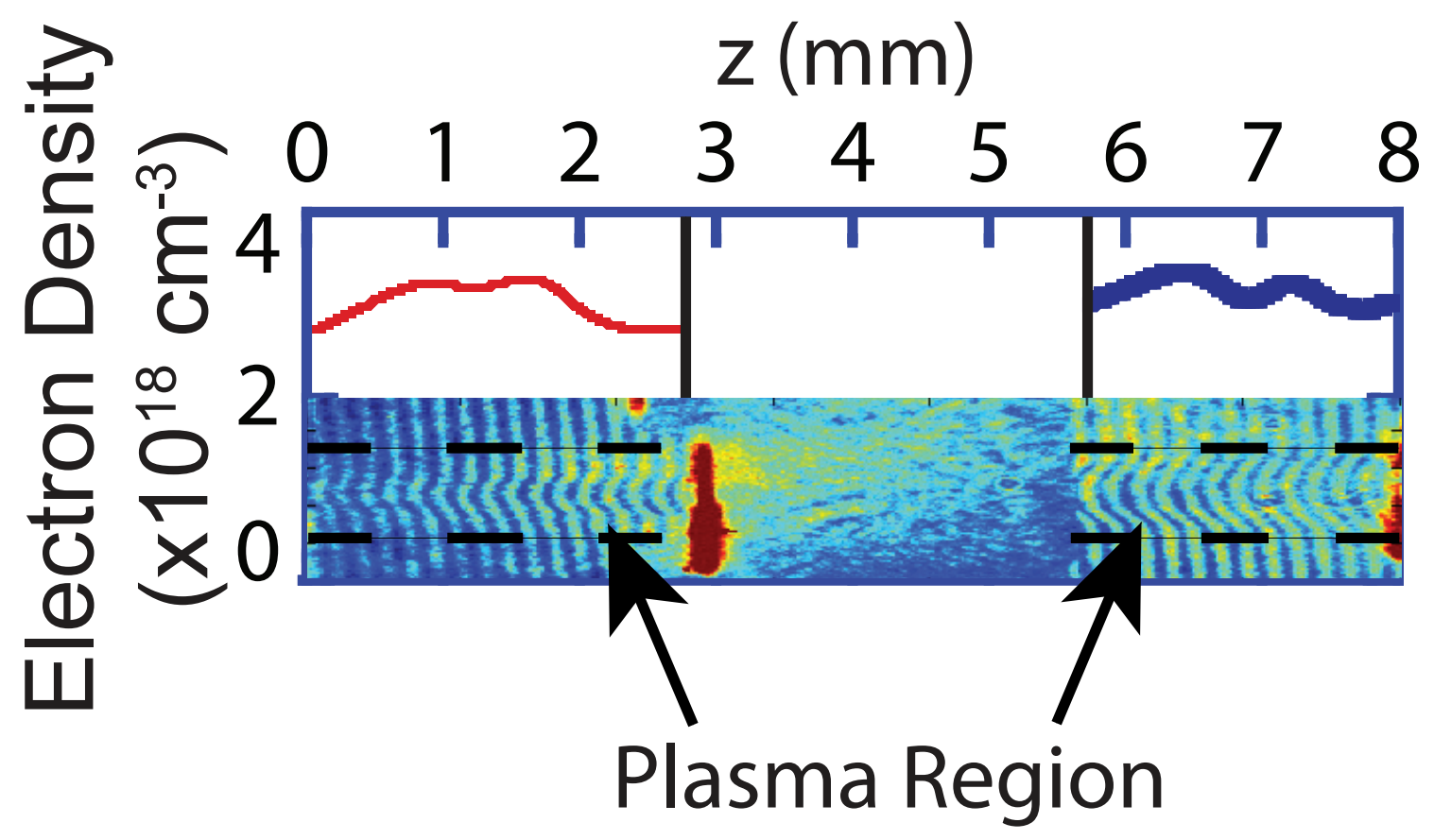

Figure 3.6: Typical inteferogram (in false color) recorded during the two-stage gas cell experiments with the corresponding Abel-inverted density profile. 
A typical interferogram from the two-stage gas cell experiments is shown in Fig.3.6, along with its corresponding Abel-inverted electron density profile. In this case the main pulse propagates from left to right, and the region between $2.8 \mathrm{~mm}$ and $5.7 \mathrm{~mm}$ is obscured by the gas cell hardware that joins the two stages together. The interferometer is aligned such that in vacuum (regions void of plasma) the arms of the interferometer interfere to produce straight, parallel fringes at the plane of the CCD camera that records the interferogram. When the main arm of the probe traverses a medium with an index of refraction not equal to 1 (as in the reference arm) there is a phase shift relative the surrounding region. The amount of this phase shift $\Delta \phi$ introduced by the plasma is proportional to the electron density and the chord length through the plasma by

$$
\Delta \phi=\int \Delta k d l
$$

where $\Delta k=k_{0}-k, k_{0}=2 \pi / \lambda_{0}$, and $k$ is determined by the dispersion relation for light in a plasma $\omega^{2}=k^{2} c^{2}+\omega_{p}^{2}$. For a uniform, cylindrically symmetric, underdense $\left(\omega \approx \omega_{0}\right)$ plasma this becomes

$$
\Delta \phi=\frac{2 \pi n_{e}}{\lambda_{0} 2 n_{c}} l
$$

where $l$ is the chord length. For non-uniform plasmas, Abel's inversion[34] provides a recipe for determining the radial density profile by building up the density profile one chord at a time. At each longitudinal position along the interferogram an Abel inversion is performed, and the density on-axis is reported as the plasma density which is of interest.

\subsubsection{Spectroscopy}

The plasma emission is 1:1 imaged onto the entrance slit of a $1 / 3-\mathrm{m}$ spectrometer coupled to a 16-bit CCD camera. Light is collected along the same path as the second arm of the interferometer, and the $800 \mathrm{~nm}$ light from the probe (which is substantially brighter than the plasma emission) is dispersed out of the CCD camera field of view by the spectrometer. The system has a spectral resolution of 
$2.5 \AA$ and the same spatial resolution and field of view as the interferometer; the spectral field of view is $65 \mathrm{~nm}$. This diagnostic was only used during the two-stage experiments, and is discussed further in that chapter.

\subsubsection{Transmitted Laser Spot Imaging}

A $5 \mu \mathrm{m}$ thick pellicle placed downstream of the target reflects a portion of the transmitted laser light into a forward optical diagnostic system which determines the spatial profile and spectral content of the laser pulse after traversing the plasma. As stated in the previous chapter, the laser pulse must be self-guided at nearly its focused spot size in order to maintain a sufficiently high intensity to drive a wake over centimeter-scale distances (or distances greater than 2 Rayleigh lengths, which for $\mathrm{w}_{0}=15 \mu \mathrm{m}$ corresponds to $1.75 \mathrm{~mm}$ ). Relay imaging the exit plane of the target onto a CCD camera allows the laser spot size at the exit of the plasma to be measured, and it is found that in order to accelerate electrons above $10 \mathrm{MeV}$ some degree of self-guiding must occur. A typical image of a self-guided laser pulse is shown in Figs. 6.4a 6.4b.

\subsubsection{Transmitted Laser Spectrum}

In addition to measuring the spatial profile of the laser beam at the plasma exit, its spectral content is also measured by relay imaging the exit of the target into either a spectrometer or a prism. While there is not a quantitative theory relating the distance over which the laser pulse drives a wake to the extent of the observed red-shifting due to energy loss to driving the wake and photon conservation, we are able to compare shot-to-shot transmitted beam spectra and comment on the extent of the red-shift and the plasma length. For observing red-shifts out to less than $1 \mu \mathrm{m}$ wavelength the transmitted light is focused onto the entrance slit of a $1 / 3-\mathrm{m}$ spectrometer coupled to a 16-bit CCD camera. However, we have also implemented a prism and an 8-bit infrared camera to detect red-shifts to wavelengths greater than $1 \mu \mathrm{m}$ (where our CCD cameras have a quantum efficiency of zero). We have observed light extending past $1200 \mathrm{~nm}$ in this configuration, indicating a strong 
wake driven over nearly $1 \mathrm{~cm}$ of plasma. The spectral calibration of the prism system is achieved by measuring the spectral width of the Fabry-Perot fringes induced in the spectrum by the pellicle, which can be carefully measured using the optical spectrometer setup. Red-shifting observed from the prism system is shown in Figs. 6.4c and 6.4d.

Chapter 3, in part, is a reprint of the material as it appears in: B. B. Pollock, J.S. Ross, G. R. Tynan, D. H. Froula, S. H. Glenzer, V. Leurent, J. P. Palastro, J. E. Ralph, C. E. Clayton, K. A. Marsh, A. E. Pak, T. L. Wang, and C. Joshi. TwoScreen Method for Determining Electron Beam Energy and Deflection from a Laser Wakefield Accelerator. Proceedings of the 2009 Paricle Accelerator Conference, Vancouver, 2009(http://epaper.kek.jp/PAC2009/papers/we6rfp101.pdf), p. A14. The dissertation author was the primary investigator and author of this paper. 


\section{Chapter 4}

\section{Plasma Length Scaling and Self-Trapping Threshold}

The electron energy gain equation from Ch. 2, Eq. 2.26, indicates that the maximum energy gain from LWFA increases with decreasing electron density. As the density is reduced the electric field inside the wake is also reduced, but the dephasing length increases faster than the field falls off. This chapter describes a series of self-trapping experiments using three different length gas jet targets that has been carried out using the Callisto laser system which demonstrates this scaling. For each gas jet length an optimum density exists which sets the plasma length equal to the dephasing length and maximizes the energy gain. Densities below the optimum result in electron beams which have not yet dephased, while densities above the optimum correspond to electrons which dephased and began decelerating. In addition, a density threshold is determined below which the selftrapping mechanism is no longer reliable for injecting charge into the wake. The same threshold behavior is also observed in simulations. 


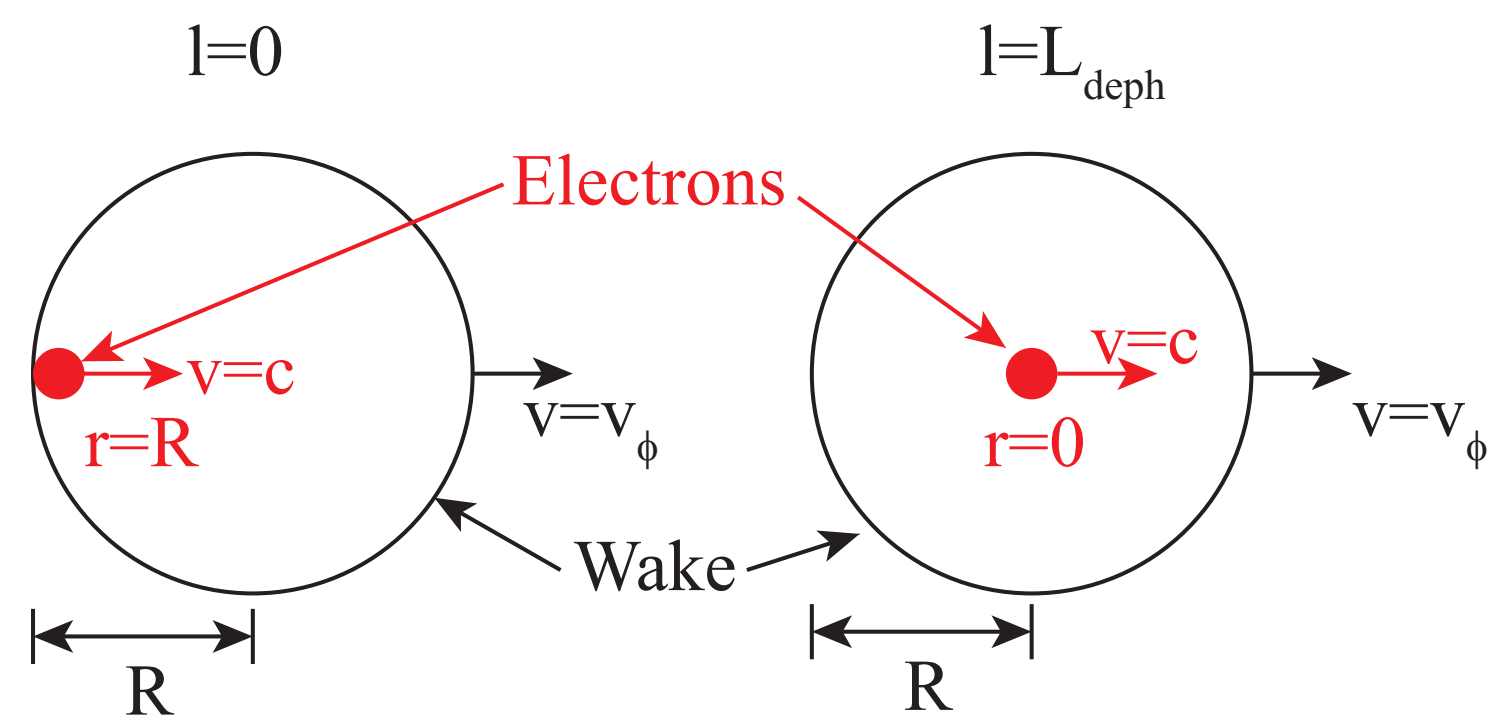

Figure 4.1: Shown on the left are electrons trapped at the back of the first bucket of the wake at a radius $\mathrm{R}$ from the center of the wake. The laser has been omitted in this figure, but propagates to the right inside the wake (as in Fig. 2.1). The trapping is assumed here to occur at the start of the plasma, corresponding to a laser propagation length $\mathrm{l}=0$ in the plasma. The electrons propagate to the right at essentially c, while the the wake (and laser) propagates at the reduced speed $\mathrm{v}_{\phi}$ determined by Eq. 2.24. On the right is the case corresponding to the laser having propagated one dephasing length in the plasma, where the electrons have now reached the position $\mathrm{r}=0$ and the zero of the longitudinal electric field (see Fig. 2.2). 


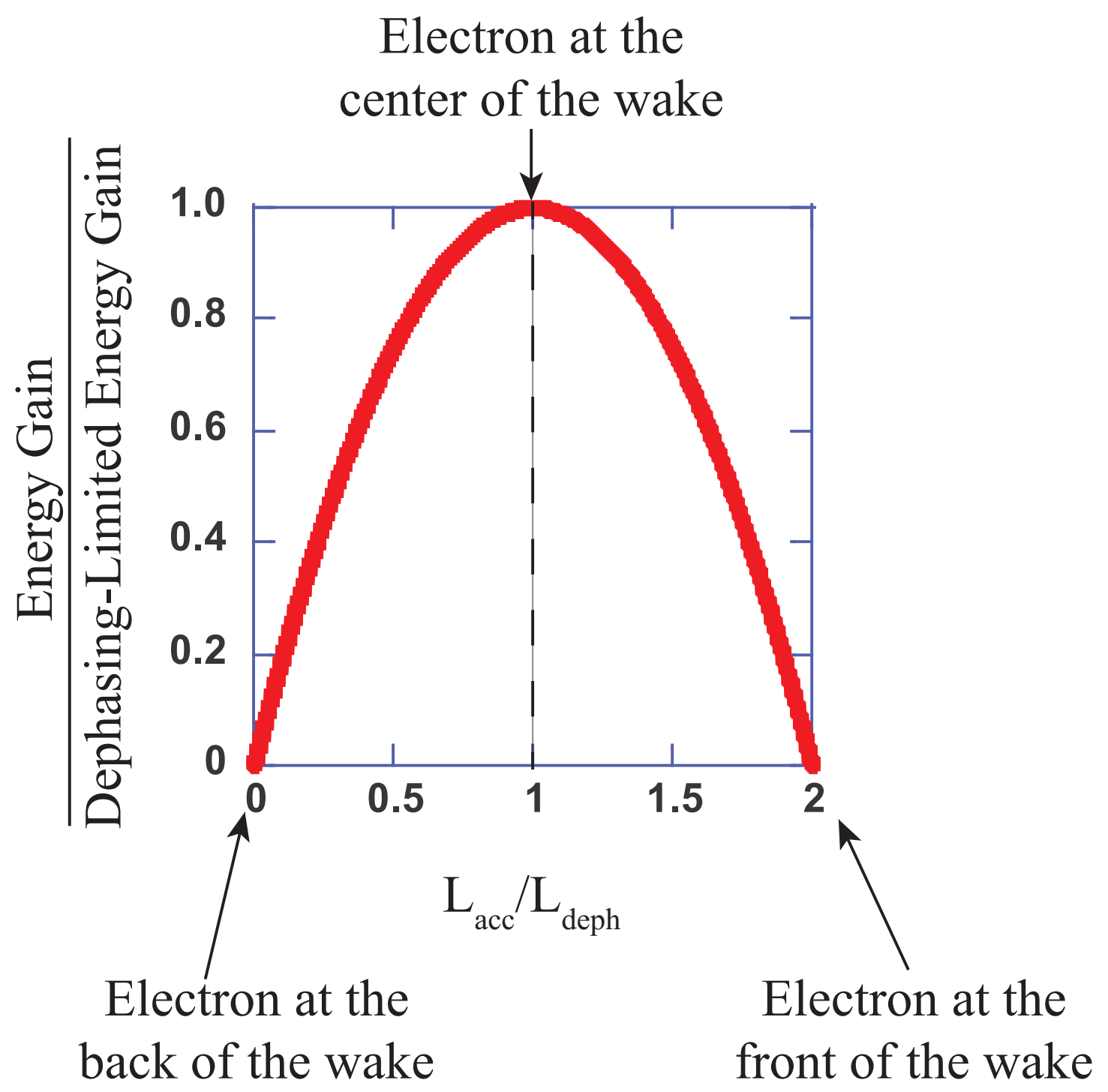

Figure 4.2: The energy gain calculated by Eq. 4.4 is normalized to the dephasing limited energy gain from Eq. 2.26 and plotted against the acceleration length normalized to the dephasing length. When $l_{a c c}=0$ the electron is at the back of the bucket. As the laser propagates the electron moves toward the center of the bucket; after one dephasing length in the laboratory frame the electron has moved from the back of the bucket to the center of the bucket and the zero of the longitudinal electric field. If the plasma is longer than a dephasing length the laser continues to propagate and the electron moves into the decelerating electric field portion of the wake. If an electron moved all the way to the front of the wake, corresponding to the laser propagating two dephasing lengths in the plasma, the net energy gain for the electron goes to zero for a symmetric electric field. 


\subsection{Electron Energy Gain Over a General Accel- erating Length}

The maximum energy gain that can be achieved from LWFA when the length of the plasma is equal to the dephasing length for the corresponding electron density is determined by Eq. 2.26. This equation does not describe, however, the energy gain if the density is too high (low), corresponding to a plasma length that is longer (shorter) than the dephasing length. For this more general calculation the energy gain is determined by the integral of the electric field over the length of the plasma

$$
W=-\int_{0}^{L_{a c c}} e \mathbf{E} \cdot \mathbf{d} \mathbf{l}
$$

where $L_{a c c}$ is the plasma length over which electrons accelerate in the wake electric field. When $L_{a c c}$ is set to $L_{\text {deph }}$ Eq. 4.1 takes the same form as Eq. 2.26. Substituting the wake electric field from Eq. 2.14 (with the simulatio-determined coefficient of 1.5) into Eq. 4.1 gives

$$
W=\int_{0}^{L_{a c c}} e \frac{e}{2 \epsilon_{0}} n_{e} r d l
$$

where a change of variable from the radial coordinate in the wake frame to the longitudinal coordinate in the laboratory frame is now required. Since an electron moves from the rear of the bucket (with radius $R$ ) to a radius of zero in the wake frame while traversing one dephasing length in the laboratory frame, as illustrated in Fig. 4.1, the two systems are related by

$$
r=R\left(1-\frac{l}{L_{\text {deph }}}\right)
$$

The general expression for the energy gain is then

$$
W=\int_{0}^{L_{a c c}} \frac{e^{2}}{2 \epsilon_{0}} n_{e} r d l=\frac{e^{2}}{4 \epsilon_{0}} n_{e} R L_{d e p h}\left(2 \frac{L_{a c c}}{L_{d e p h}}-\frac{L_{a c c}^{2}}{L_{d e p h}^{2}}\right)=W_{m a x}\left(2 \frac{L_{a c c}}{L_{d e p h}}-\frac{L_{a c c}^{2}}{L_{d e p h}^{2}}\right)
$$


where electron trapping at the back of the first wake period is still assumed, $W_{\max }$ is the dephasing-limited energy gain from Eq. 2.26, and $L_{a c c}=L_{\text {deph }}$ again recovers the dephasing-limited energy gain expression. The energy gain calculated from Eq. 4.4 (normalized to $W_{\max }$ ) is plotted in Fig. 4.2 as a function of the fraction of a dephasing length electrons traverse in the plasma. As expected the energy gain maximizes for a plasma length of one dephasing length, and due to the linear and symmetric electric field the energy gain is quadratic. If an electron is allowed to propagate two dephasing lengths in the wake it will have a net zero energy gain from this electric field.

\subsection{Gas Jets}

Experiments using the Callisto laser system have been performed in order to investigate the validity of Eq. 4.4. The experimental setup from Fig. 3.3 is implemented with three different diameter gas jets as targets to create three different length plasmas (these experiments did not use the plasma emission diagnostic, and the transmitted laser spectrum is measured with the spectrometer and 16-bit CCD camera system). By varying the electron density at each length the dephasing length and dephasing limited energy gain $\mathrm{W}_{\max }$ are modified and the electron spectra produced are recorded on the Fujifilm image plates after being dispersed by the dipole magnet. During these experiments $30 \%$ of the laser energy resided inside the $\mathrm{w}_{0}=15 \mu \mathrm{m}$ focal spot.

The supersonic gas jet nozzles are designed[35] to deliver uniform gas density profiles at neutral densities of He ranging from $1.5 \times 10^{18} \mathrm{~cm}^{-3}$ to $1 \times 10^{19} \mathrm{~cm}^{-3}$. The gas jet diameters are $3 \mathrm{~mm}, 5 \mathrm{~mm}$, and $8 \mathrm{~mm}$, and are supplied with up to 2000 psi of He. The gas jets are aligned such that the plane of the nozzle opening is $1 \mathrm{~mm}$ below the laser axis. To align the nozzle in the other two axes an imaging system looks directly down onto the opening of the conical nozzle. Fig. 4.3 shows the alignment technique schematically. The target chamber is pumped down to a partial vacuum of 10 Torr of air, and the $10 \mathrm{~Hz}, 250 \mathrm{~mJ}$ alignment laser is propagated into the target chamber. As the beam approaches focus the intensity 


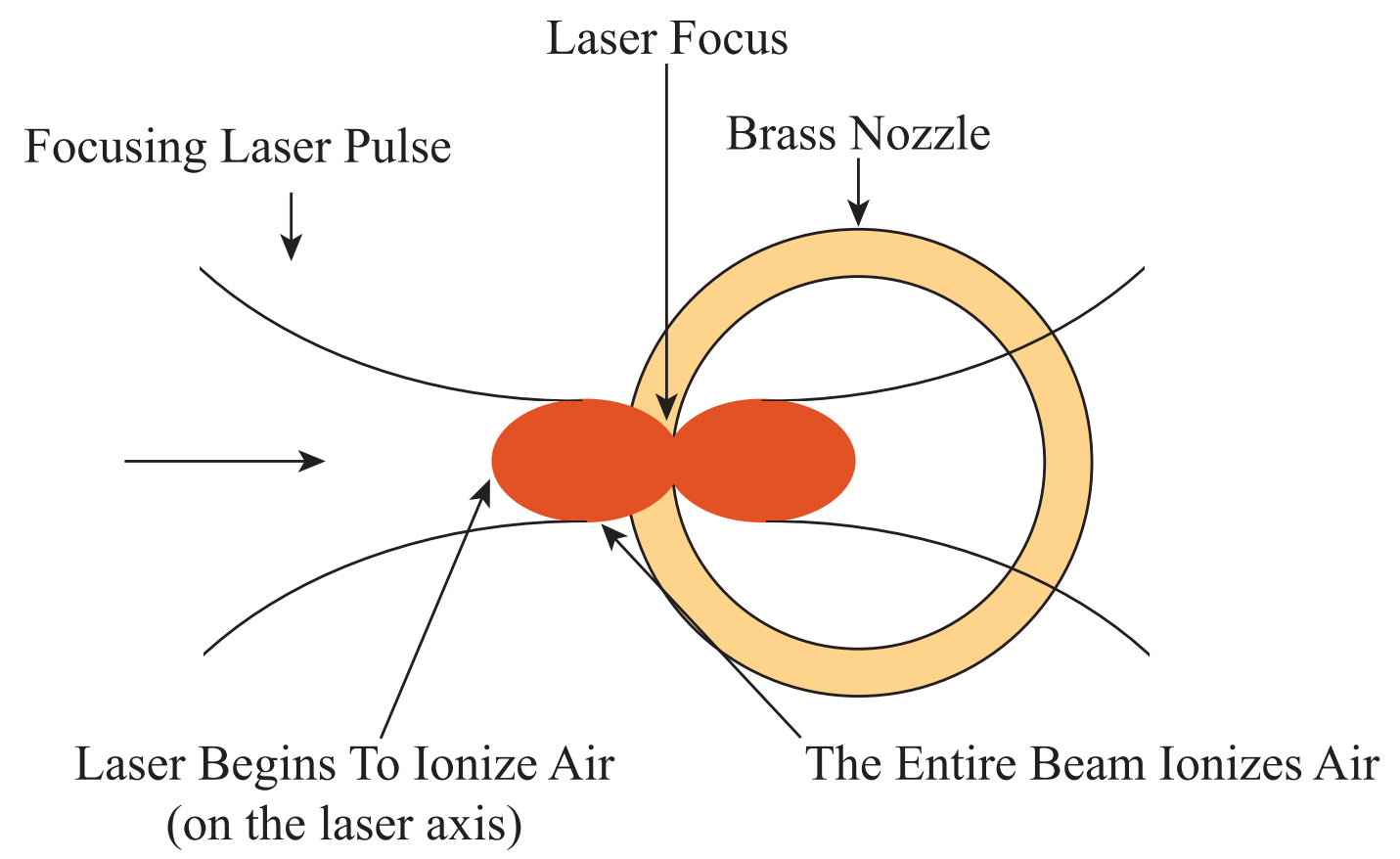

Figure 4.3: The alignment technique used to align the laser to the gas jets is shown schematically. The target chamber has been brought to a partial vacuum of 10 Torr of air. As the $250 \mathrm{~mJ}$ alignment laser (with a Gaussian transverse intensity profile) focuses the intensity on axis eventually reaches the ionization threshold for the nitrogen and oxygen molecules in the air. This gives an initially small plasma region defined by the volume of the laser pulse that is sufficiently intense to ionize the air. As the laser continues to focus the ionization radius from the laser axis becomes larger, until the whole pulse is above the ionization intensity; after this point the ionized region follows the focusing laser profile. Downstream of the focal plane the process occurs in the reverse order, and the resulting two-lobe structure has a minimum in the center corresponding to the laser focal plane. 


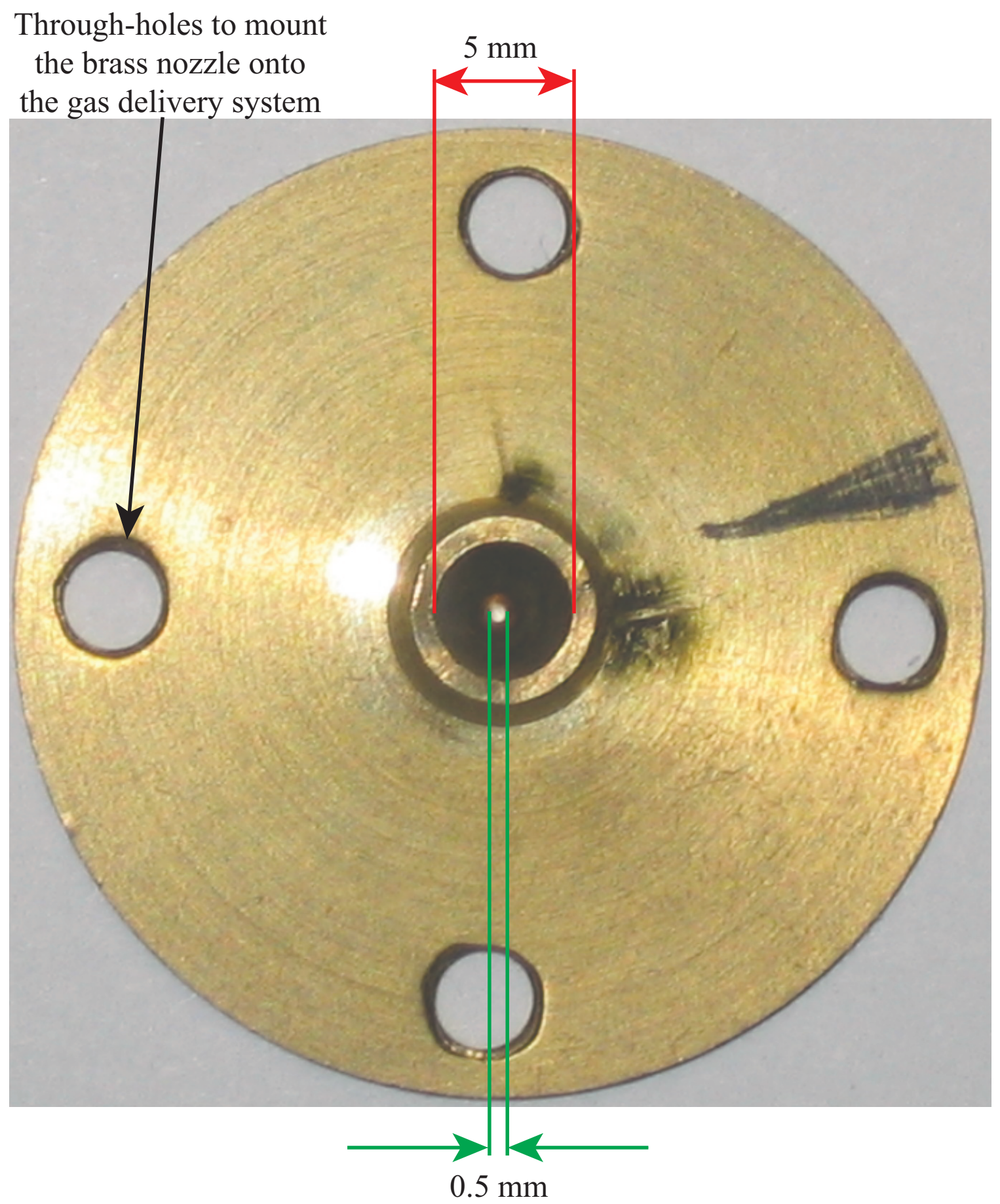

Figure 4.4: Photograph of the $5 \mathrm{~mm}$ diameter nozzle gas jet. The $3 \mathrm{~mm}$ and 8 $\mathrm{mm}$ nozzle are of similar design. 


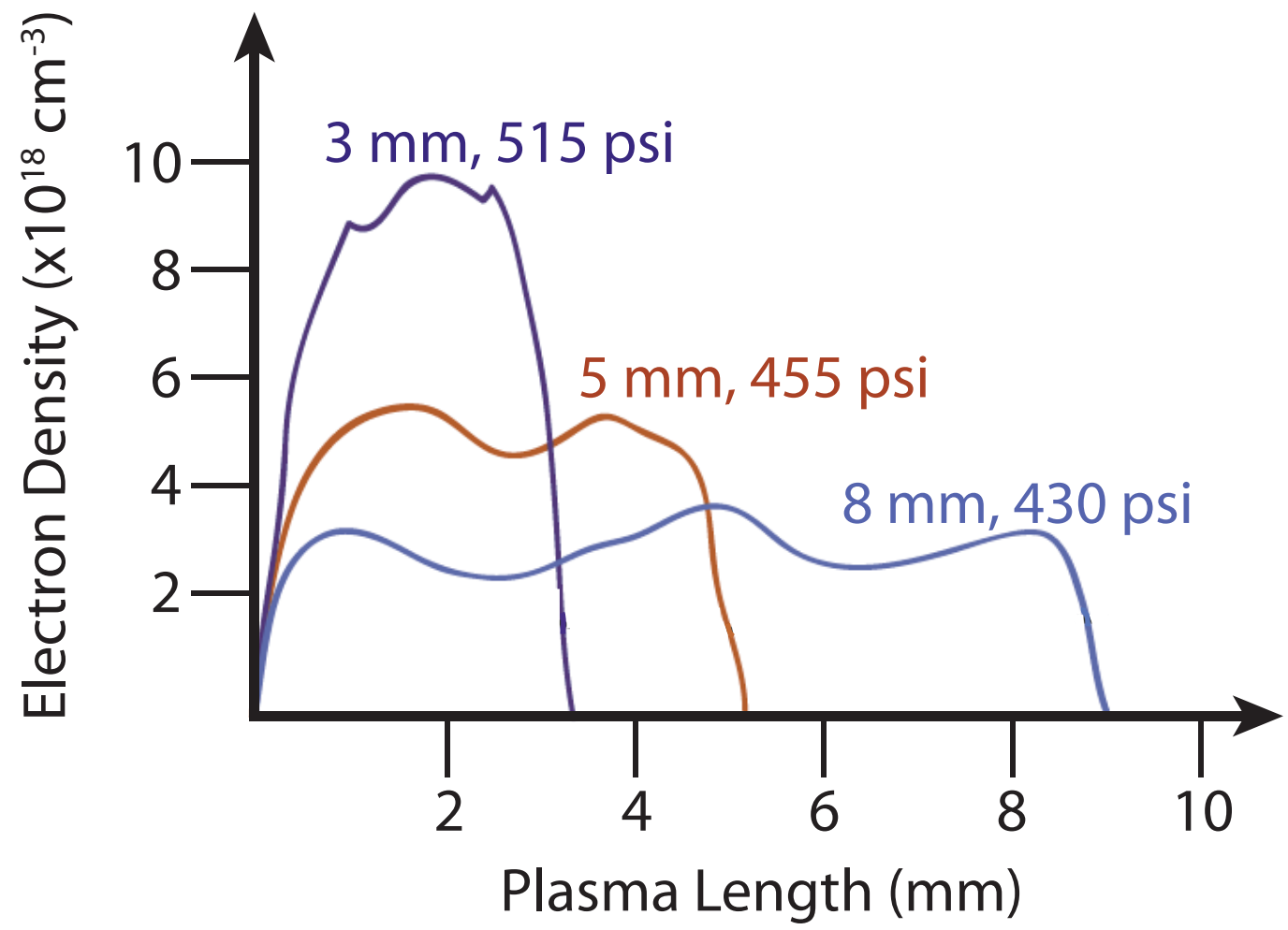

Figure 4.5: Characteristic electron density profiles for the $3 \mathrm{~mm}, 5 \mathrm{~mm}$, and 8 $\mathrm{mm}$ diameter gas jets. The backing pressure for each case is given. 
increases and eventually becomes sufficient (initially just on axis since the laser has a Gaussian transverse intensity profile) to ionize the nitrogen and oxygen molecules in the air and create a plasma. The alignment top-view system images the selfemission from this plasma as well as the gas jet nozzle. As the laser propagates further the ionization radius increases until the whole beam is above the threshold to ionize air (this is only a partial ionization); from this point to the laser focus the ionized region follows the laser profile. After focus the same process occurs in the reverse order until the laser intensity falls below the ionization threshold. Motorized translation stages allow the nozzle location to be adjusted under vacuum, and the interior edge of the upstream side of the nozzle is placed below the laser focus, which occurs at the center of the two-lobe plasma emission pattern.

A photograph of the $5 \mathrm{~mm}$ gas jet nozzle is shown in Fig. 4.4; the $3 \mathrm{~mm}$ and $8 \mathrm{~mm}$ nozzles are of the same design. Fig. 4.5 shows experimentally observed electron density profiles for each gas jet length. The electron density in each case is measured with interferometry during a high power laser shot and determined by applying Abel's inversion[34] to the interferogram; there is little shot-to-shot fluctuation in the longitudinal density profile. The electron density and backing pressures for each profile are given in Fig. 4.5.

\subsection{Evidence of Wake Formation}

While interferometry measurements indicate plasma formation, they do not imply wake production. In order to diagnose whether or not a wake is driven the transmitted laser diagnostics discussed in Ch. 3 are necessary; these provide information about the degree of self-guiding that occurs in the plasma and the extent of the red-shifting to the laser spectrum that results from wake generation. The layout of the transmitted laser diagnostics is shown in Fig. 4.6.

Representative data for the laser self-guiding is shown in Ch. 6. During the experiments described in the present chapter, in each case where electrons were trapped and accelerated in the wake the laser spot size at the exit of the plasma was with a factor of two of the focal spot size. For the $8 \mathrm{~mm}$ gas jet this 


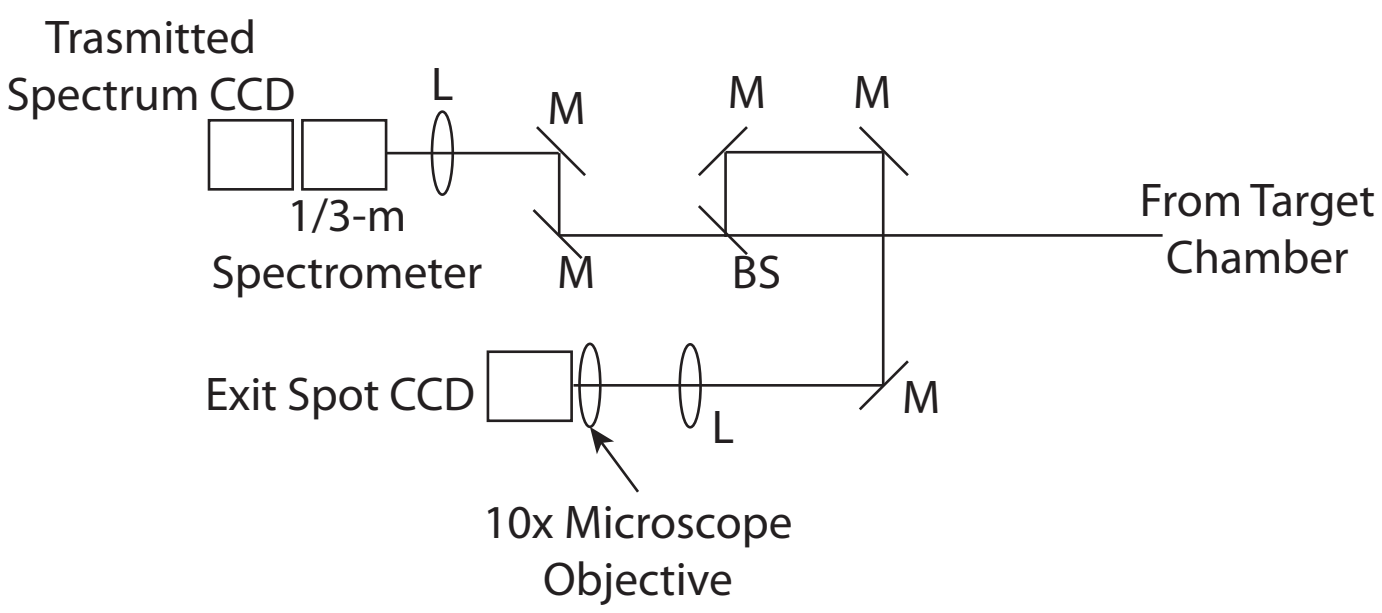

Figure 4.6: Laser light reflected by the pellicle inside the target chamber is collimated by a lens before exiting the target chamber. The collimated light is partially reflected by a beamsplitter into the exit spot imaging system, while the transmission through the beamsplitter is directed into the transmitted spectrum system. The spot size diagnostic consists of a foucsing lens that creates a 1:1 image of the plasma exit, which is magnified by a $10 \mathrm{x}$ microscope objective onto a 14-bit CCD camera. The system yields $0.7 \mu \mathrm{m} /$ pixel magnification with a resolution of $\approx 20 \mu \mathrm{m}$. The spectral diagnostic also creates a 1:1 image of the plasma exit plane, but here at the entrance slit to a $1 / 3-\mathrm{m}$ spectrometer that is coupled to a 16 -bit CCD camera. This spectral dispersion is $0.27 \mathrm{~nm} /$ pixel and has a spectral field of view of $280 \mathrm{~nm}$. 
corresponds to self-guiding over more than 10 Rayleigh lengths. Representative spectral data is shown in Ch. 6, where the spectrum is better resolved and the red-shifting extends further due to the increased plasma length in the experiments described therein. During the gas jet experiments a degree of red-shifting is always present when electrons are observed on the image plates.

\subsection{Electron Energy Scaling}

Electron spectra from each of the three gas jet targets have been recorded for electron densities ranging from $3 \times 10^{18} \mathrm{~cm}^{-3}$ to $9 \times 10^{18} \mathrm{~cm}^{-3}[14,36]$. The maximum electron beam energy recorded during these experiments is $720 \mathrm{MeV}$, which corresponds to an electron density of $3 \times 10^{18} \mathrm{~cm}^{-3}$ using the $8 \mathrm{~mm}$ gas jet. Fig. 4.7 shows the raw electron spectra on the first image plate for the highest energy electrons recorded using each gas jet. The laser power coupled into the central spot is 65 TW in each case.

The dephasing length is related to the plasma density and laser power through Eq. 2.25; the dephasing length for a fixed coupled power of $65 \mathrm{TW}$ is plotted in Fig. 4.8, as are the electron densities that correspond to the peak electron energies observed with each gas jet length. The dephasing-limited energy gain $\mathrm{W}_{\max }$ from Eq. 2.26 for the same conditions is plotted in Fig. 4.9, as well as the maximum energy achieved in each gas jet at the corresponding density. The horizontal error bars in each of these figures are from the uncertainty in the interferometry analysis; the energy error bar is from the uncertainty in determining the position of the electron beam on the image plates.

Comparing the results of Fig. 4.8 and Fig. 4.9 with the prediction from Fig. 4.2 there is a discrepancy between the expected energy and the maximum observed. The $3 \mathrm{~mm}, 5 \mathrm{~mm}$, and $8 \mathrm{~mm}$ gas jets are $1.93 \mathrm{~mm}, 3.165 \mathrm{~mm}$, and $3.375 \mathrm{~mm}$ longer, respectively, than the dephasing lengths for the densities which maximized the energy gain in each jet. From Eq. 4.4 the maximum energy gain in the $8 \mathrm{~mm}$ jet should have been only $349 \mathrm{MeV}$, while the other two jets were more than twice the dephasing length for the densities in question. To explore this 


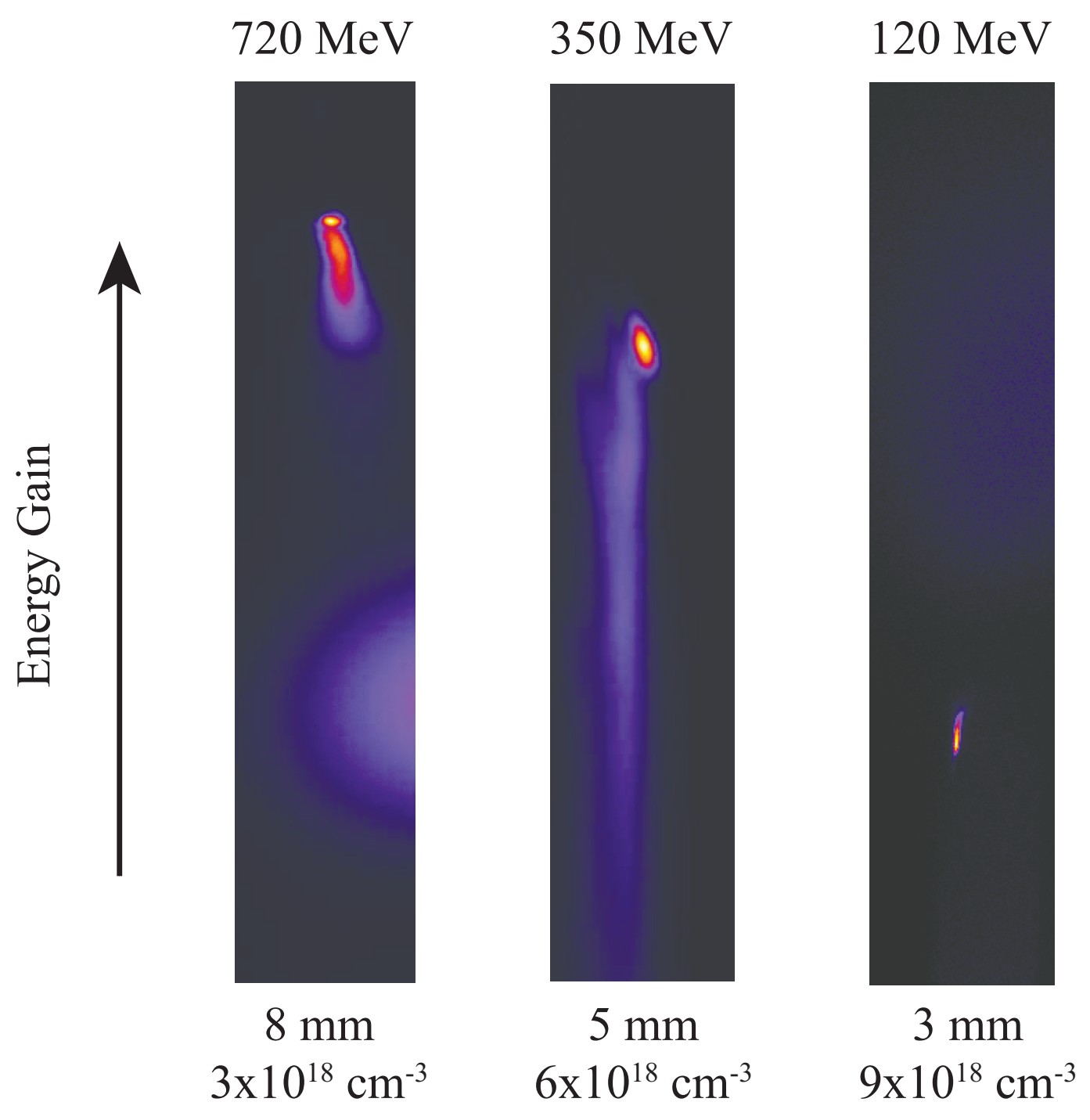

Figure 4.7: Raw dispersed electron spectra from the first image plate are shown for the maximum electron energy achieved in each of the gas jets. The laser power in each case is $65 \mathrm{TW}$. 


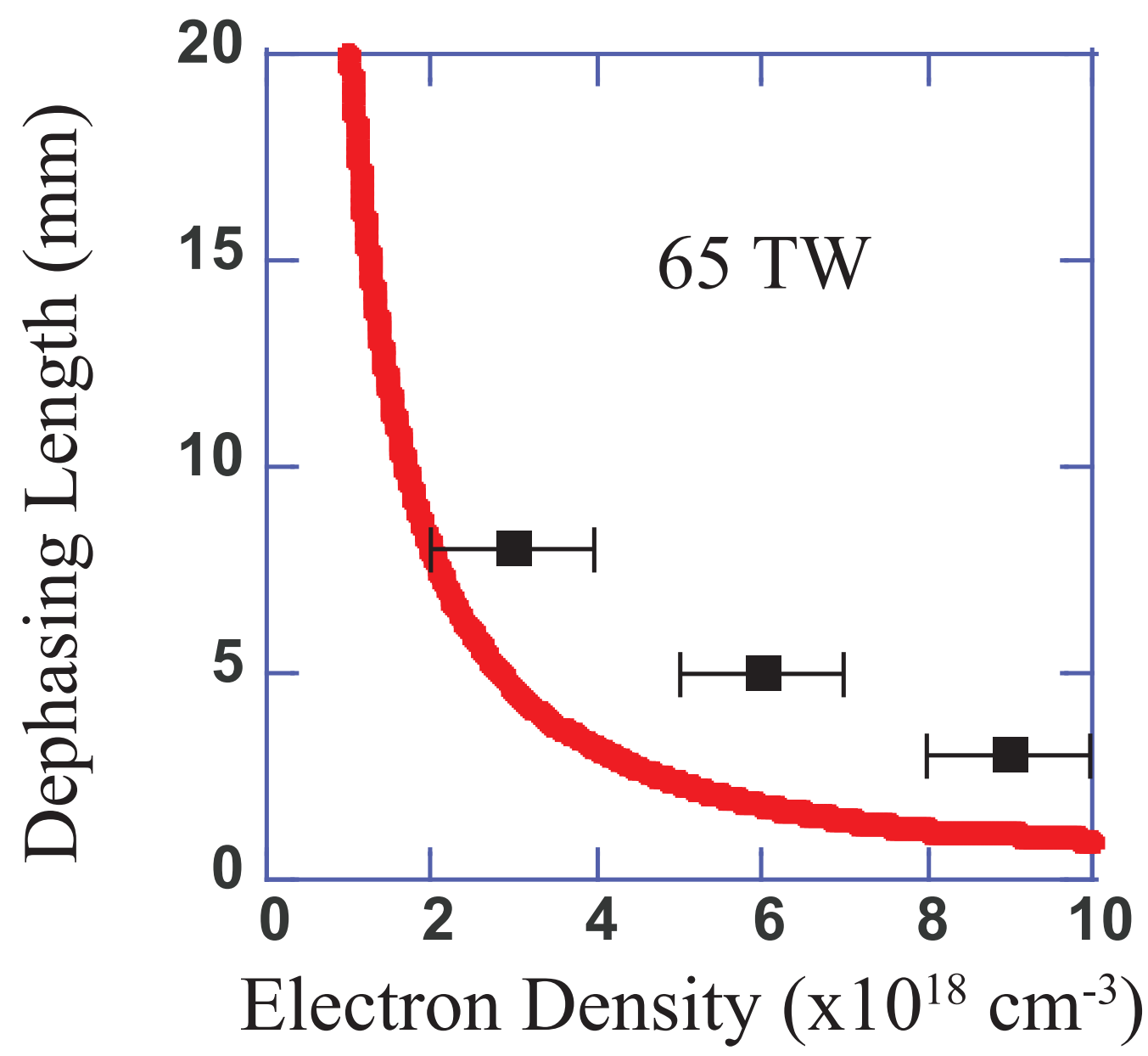

Figure 4.8: The dephasing length calculated in Eq. 2.25 for a laser power of 65 TW is shown in red. The black squares correspond to the electron density which maximized the electron energy for each gas jet length. 


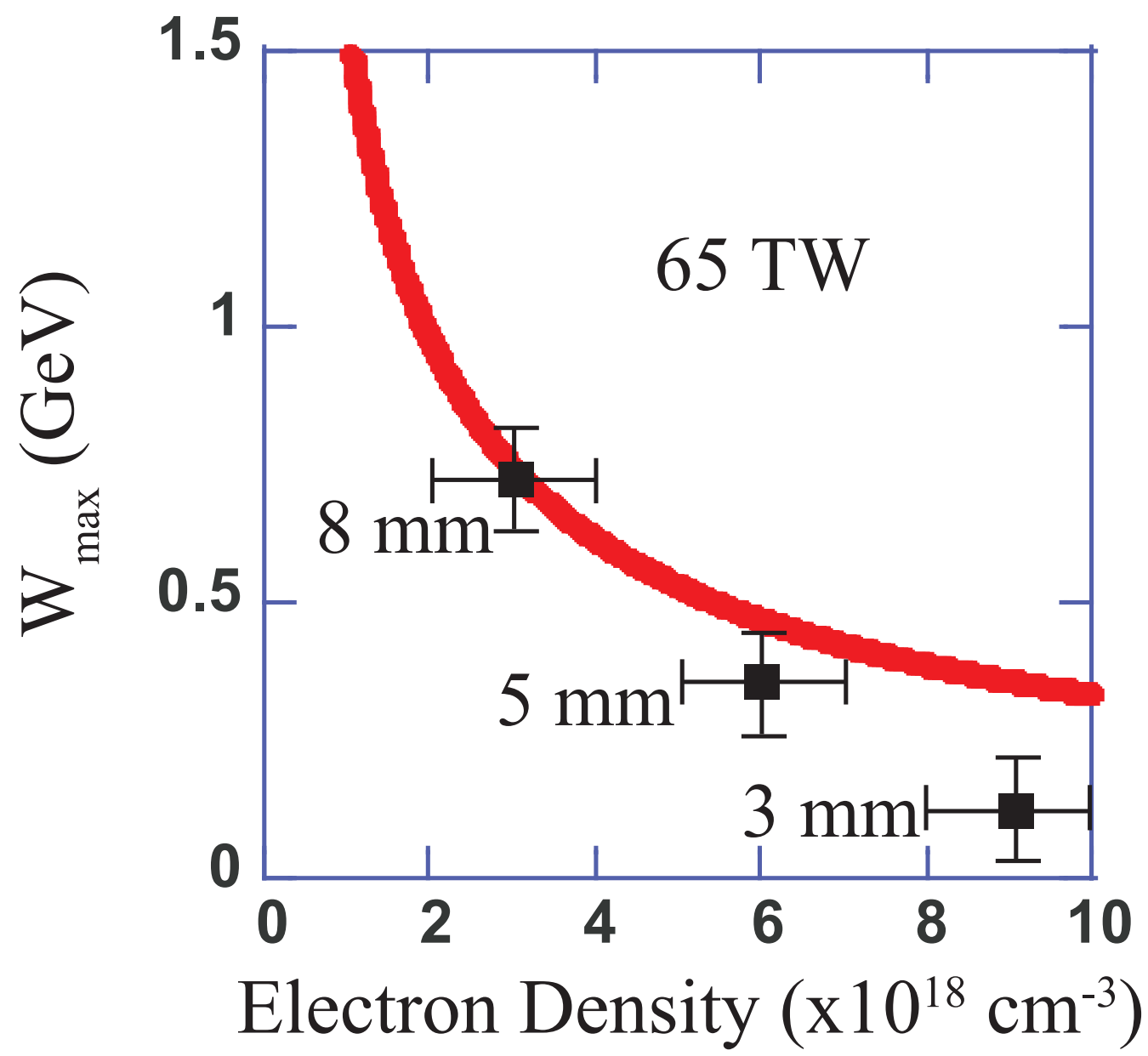

Figure 4.9: The dephasing-limited energy gain calculated in Eq. 2.26 for a laser power of $65 \mathrm{TW}$ is shown in red. In addition, the black squares indicate maximum energy achieved in each gas jet at the corresponding electron density. 


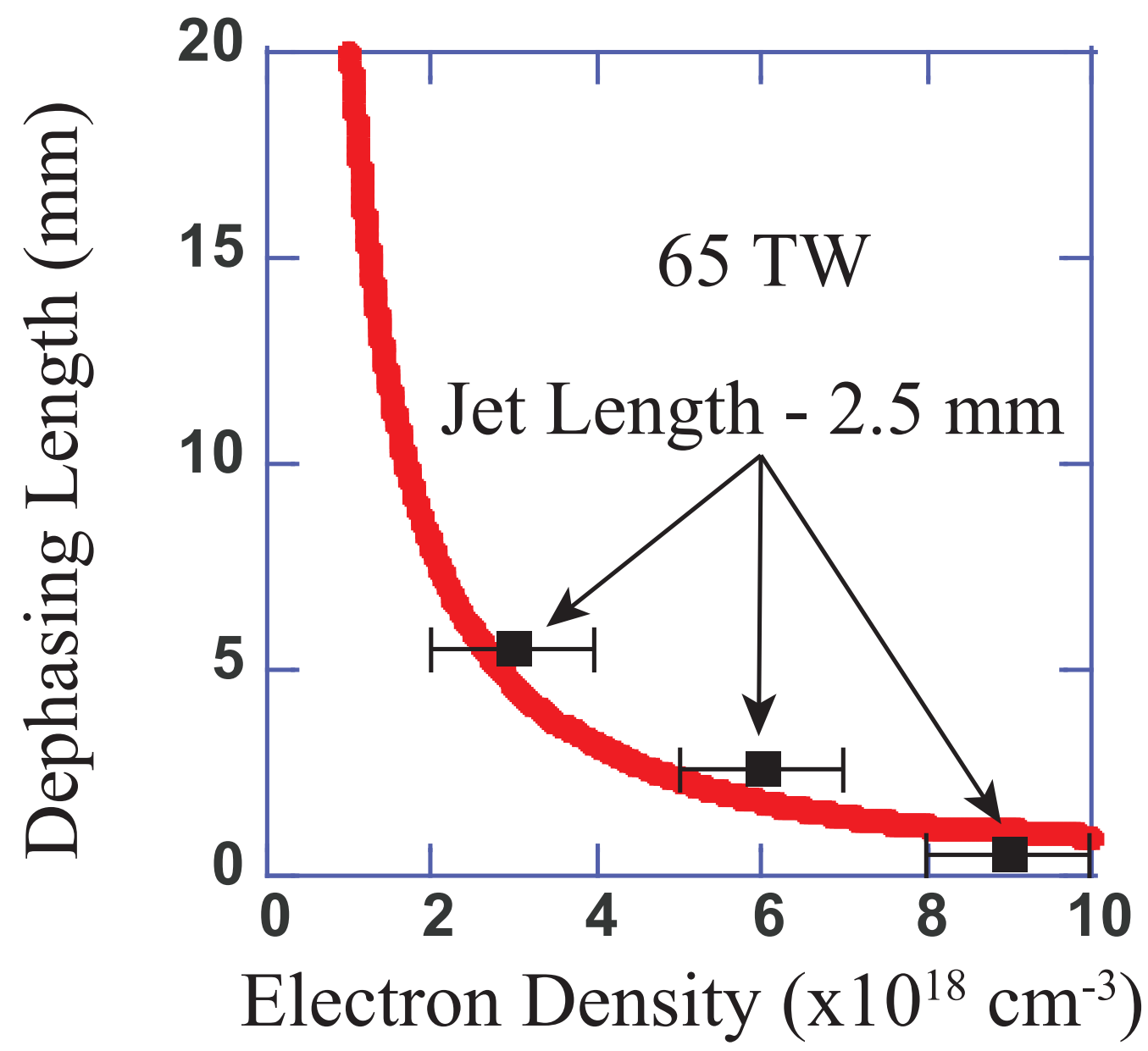

Figure 4.10: The dephasing length calculated in Eq. 2.25 for a laser power of 65 TW is shown in red. The black squares indicate the useful length of the plasma for each gas jet length at the density corresponding to the maximum recorded electron energy. 


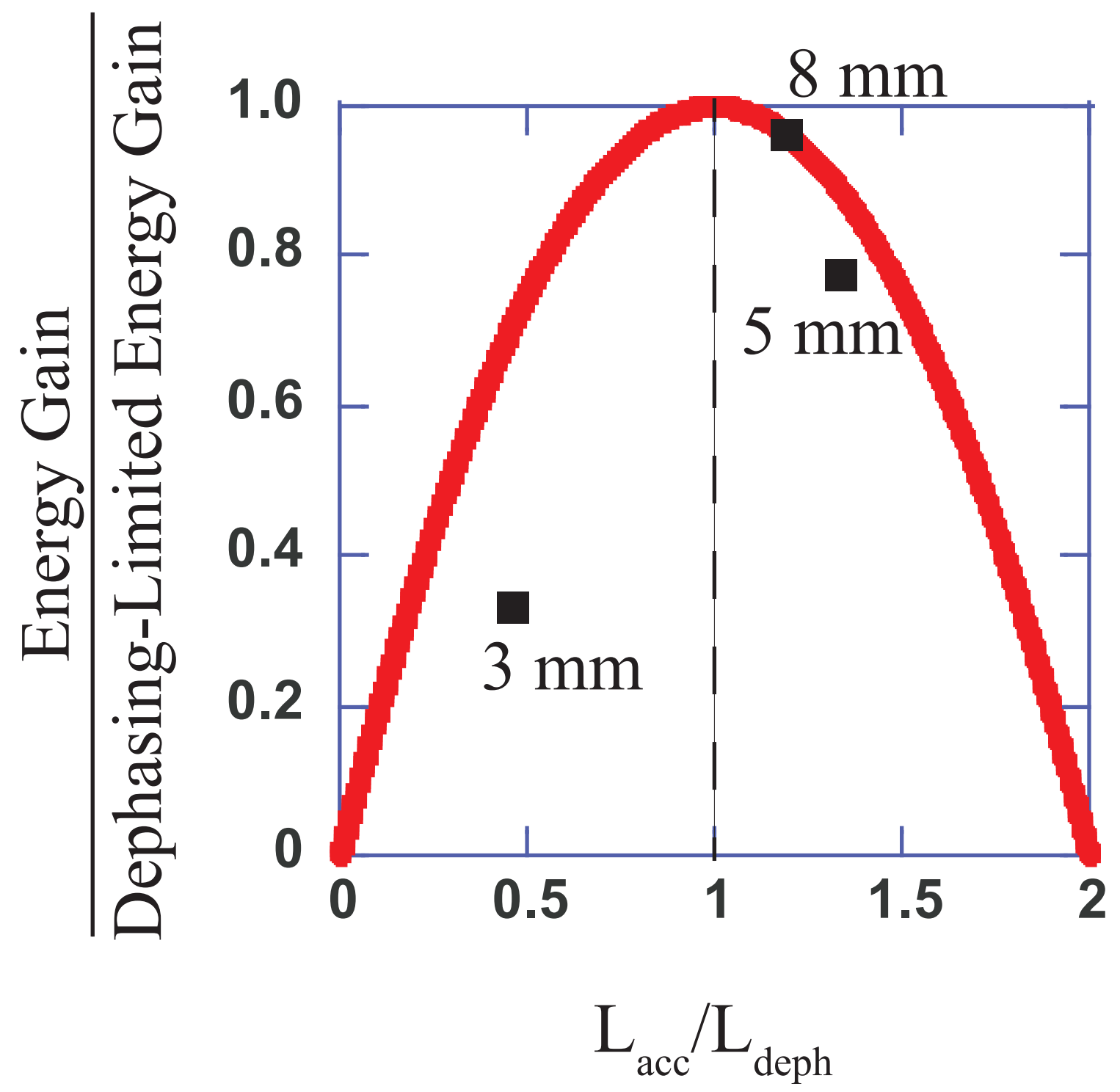

Figure 4.11: Eq. 4.4 is plotted with the experimental data corresponding to the maximum observed electron energy from each gas jet. 
further simulations using the PIC code OSIRIS[28] were employed to examine the LWFA process for the conditions of these experiments.

As discussed in Ch. 2, the blowout regime is optimized when the laser spot size $\mathrm{w}_{0}$ is close to the blowout radius from Eq. 2.15. The spot size at Callisto is $15 \mu \mathrm{m}$, while the blowout radius for 3,6 , and $9 \times 10^{18} \mathrm{~cm}^{-3}$ is $11.9,9.4$, and 8.2 $\mu \mathrm{m}$, respectively, for the laser parameters of these experiments. The simulations indicate that while the laser is self-guided immediately in the plasma (since $\mathrm{P}$ exceeds $\mathrm{P}_{\text {crit }}$ ) a propagation distance of $2.5 \mathrm{~mm}$ is required for the plasma to modify the laser spot to the matched spot size $\mathrm{R}[14]$. It is after this laser evolution length $\mathrm{L}_{e v}=2.5 \mathrm{~mm}$ that self-trapping is observed to begin, and thus to compare with theory which assumes trapping at the beginning of the plasma the evolution length must be subtracted from the total length of the plasma.

Fig. 4.10 again shows the dephasing length for a 65 TW laser pulse, but now the points corresponding to the densities which maximized electron energy for each jet include the $2.5 \mathrm{~mm}$ offset from the length of the jet as determined from the OSIRIS simulations. The points are now in much better agreement with the calculated dephasing length. Comparing again with Eq. 4.4 the $8 \mathrm{~mm}$ gas jet (with a useful length of $5.5 \mathrm{~mm}$ ) is 1.19 times the dephasing length for a density of $3 \times 10^{18} \mathrm{~cm}^{-3}$ and $65 \mathrm{TW}$; the resulting energy gain is expected to be $96.4 \%$ of the dephasing-limited energy gain $\mathrm{W}_{\max }=745 \mathrm{MeV}$, which is $718 \mathrm{MeV}$. The 5 $\mathrm{mm}$ jet is 1.36 times the dephasing length for $6 \times 10^{18} \mathrm{~cm}^{-3}$, corresponding to an energy gain $W=0.869 \times 470 \mathrm{MeV}=408 \mathrm{MeV}$. Finally, the $3 \mathrm{~mm}$ jet operating at $9 \times 10^{18} \mathrm{~cm}^{-3}$ is 0.47 times the dephasing length, which should result in a maximum possible energy of $257 \mathrm{MeV}$. These results are summarized in Fig. 4.11.

Callisto suffers from significant shot-to-shot fluctuations in the laser performance. As a result it can take many high-power system shots to conclude a parameter scan. Less than $10 \%$ of the data taken during these experiments used the $3 \mathrm{~mm}$ gas jet; the $3 \mathrm{~mm}$ gas jet is the furthest from the expected energy gain calculated with Eq. 4.4. The remainder of the data is nearly equally distributed between the $5 \mathrm{~mm}$ and $8 \mathrm{~mm}$ gas jets; the maximum energy achieved with the 8 $\mathrm{mm}$ jet is exactly as predicted by Eq. 4.4, while the maximum energy from the 5 


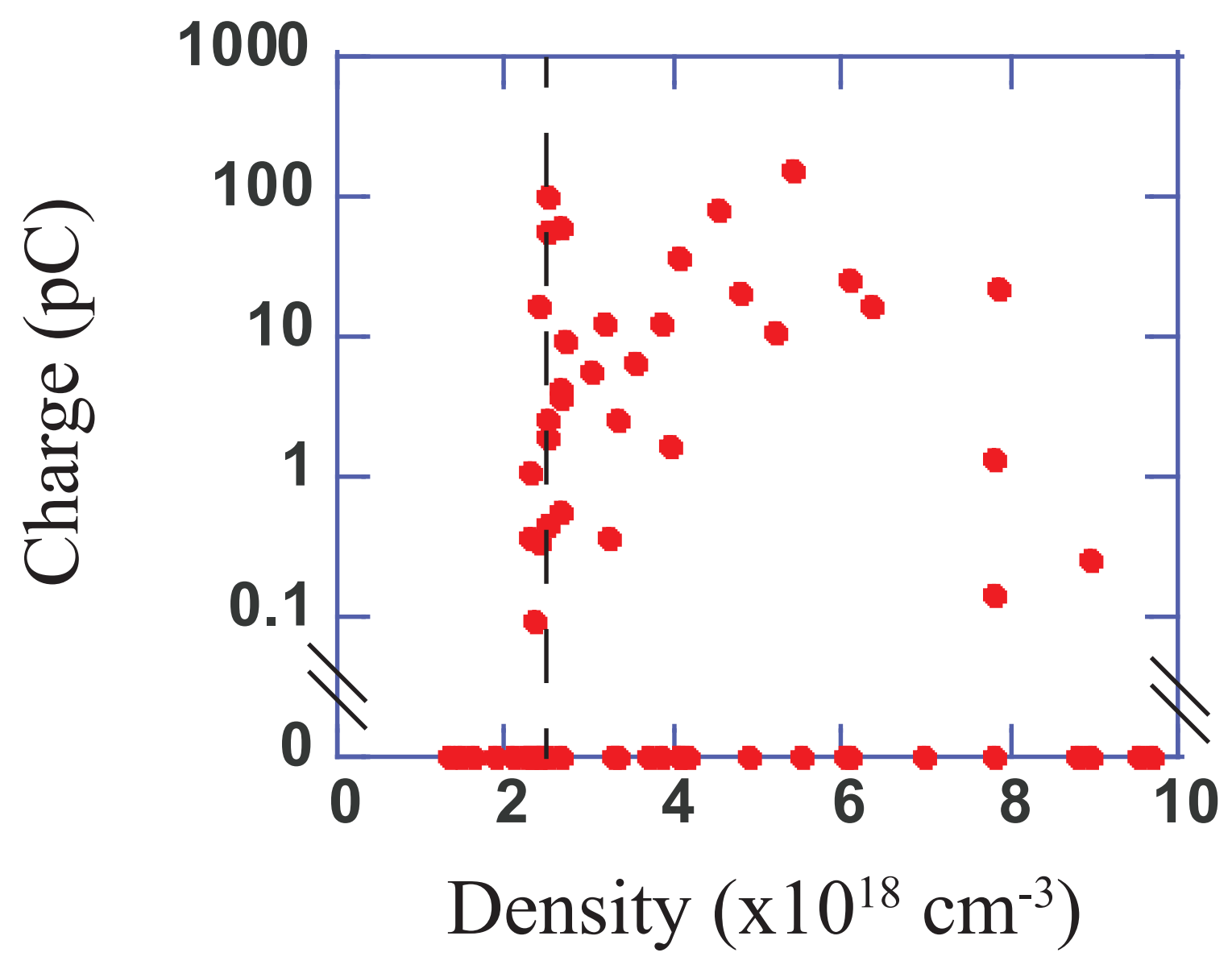

Figure 4.12: The charge observed on all high-power system shots. The vertical dashed line at $2.5 \times 10^{18} \mathrm{~cm}^{-3}$ represents the lowest density where reliable selfinjection is observed in these experiments.

$\mathrm{mm}$ jet is within $15 \%$ of the predicted value.

\subsection{Trapping Threshold}

In addition to showing that electron energy gain scales with electron density and accelerating length as predicted by the theory of the blowout regime laid out in Ch. 2, a second significant finding came out of this same set of experiments regarding the nature of the self-trapping mechanism. Fig. 4.12 shows the total charge observed during all of the high-power system shots as a function of the electron density. The charge is determined by the calibration of the Fujifilm 
image plates performed by Tanaka et. al.[33]. The self-trapping of background He electrons becomes unreliable for electron densities below $2.5 \times 10^{18} \mathrm{~cm}^{-3}$ for the laser parameters of these experiments. Several attempts were made to reduce the plasma density below this threshold, but resulted in a lack of charge recorded by the image plates. This behavior has also been observed in the OSIRIS simulations mentioned in the previous section[14], where instead of density the ratio of $\mathrm{P} / \mathrm{P}_{\text {crit }}$ is the dependent variable. As stated in Ch. 2 this density is above the required density to accelerate electrons to energies exceeding $1 \mathrm{GeV}$ in the blowout regime. The following chapter describes a series of experiments using a gas cell target, rather than gas jets, and a new charge injection mechanism that has demonstrated electron trapping at densities below $1.5 \times 10^{18} \mathrm{~cm}^{-3}$.

As a final comment, the existence of a threshold for self-trapping is perhaps not unexpected once the physical picture is examined again. As the electron density is reduced the laser group velocity, and hence the wake phase velocity, increases. This requires an electron to acquire more kinetic energy in order to be trapped by the wake. In addition to the wake velocity increasing, the bubble radius also increases as density is reduced. The theory developed by Lu et. al.[1] assumes a perfect laser pulse profile, which is never realized in the laboratory. For the larger blowout radii there is an increased chance for the electron trajectories at the back of the first bucket to miss the perfect overlap assumed by the theory, which smears out the magnitude of the electric field spike (see Fig. 2.2) at the rear of the bucket. The combination of an increased probability to smear out the spike and the increased kinetic energy required to trap then lead intuitively to a breakdown of the self-trapping mechanism, which is observed in both experiments and simulations. 


\section{Chapter 5}

\subsection{GeV Electrons from Ionization-Induced Injection}

In the previous chapter the electron beam energy produced from LWFA was shown to scale as predicted by the theory of Lu et.al. As the electron density is reduced and the plasma length increased the electron energy increases, where the maximum possible energy gain is set by the dephasing length for electrons in the wake. Additionally, it was demonstrated that the self-trapping mechanism became unreliable for injecting electrons into the wake at densities above the requirement for $>1 \mathrm{GeV}$ electron energy gain.

This chapter will describe a different injection mechanism, termed ionizationinduced injection $[15,16]$, which is able to inject charge into the wake at densities below $1.5 \times 10^{18} \mathrm{~cm}^{-3}$. A new gas cell target platform is also introduced, which allows uniform plasma densities to be produced over $>1 \mathrm{~cm}$ distances at these low densities. The result is the demonstration of $1.45 \mathrm{GeV}$ electrons, the highest energy electrons ever produced from LWFA, at the end of the tail of a broadband electron energy spectrum.

\subsection{Gas Cell Target}

Measurements of the electron density profiles in the gas jet plasmas of the previous chapter showed that producing uniform plasma profiles below $2 \times 10^{18} \mathrm{~cm}^{-3}$ 


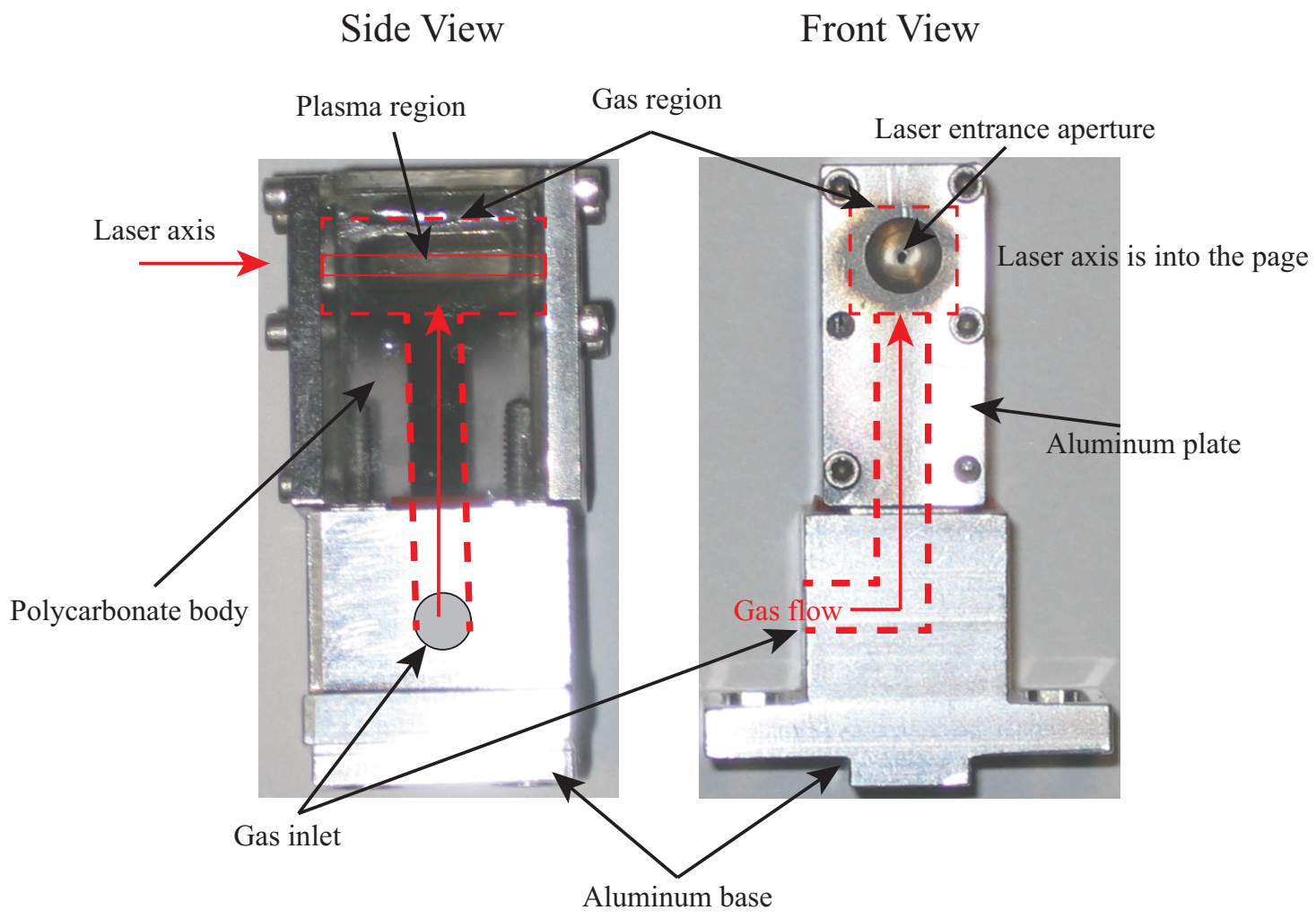

Figure 5.1: A photograph of the gas cell target. The aluminum base mounts to a set of motorized translation stages which allow the gas cell to be positioned under vacuum. The body is made of polycarbonate to allow an optical axis transverse to the laser axis for interferometry. An aluminum plate is attached to the front (back) of the polycarbonate body to seal the end of the cell; this plate contains a $500 \mu \mathrm{m}(2 \mathrm{~mm})$ aperture for the laser to propagate through the cell and for the electrons to exit the cell. The gas supply line is built into the aluminum base and the polycarbonate body, where a rubber gasket with a hole is placed between the two to form a gas seal. 


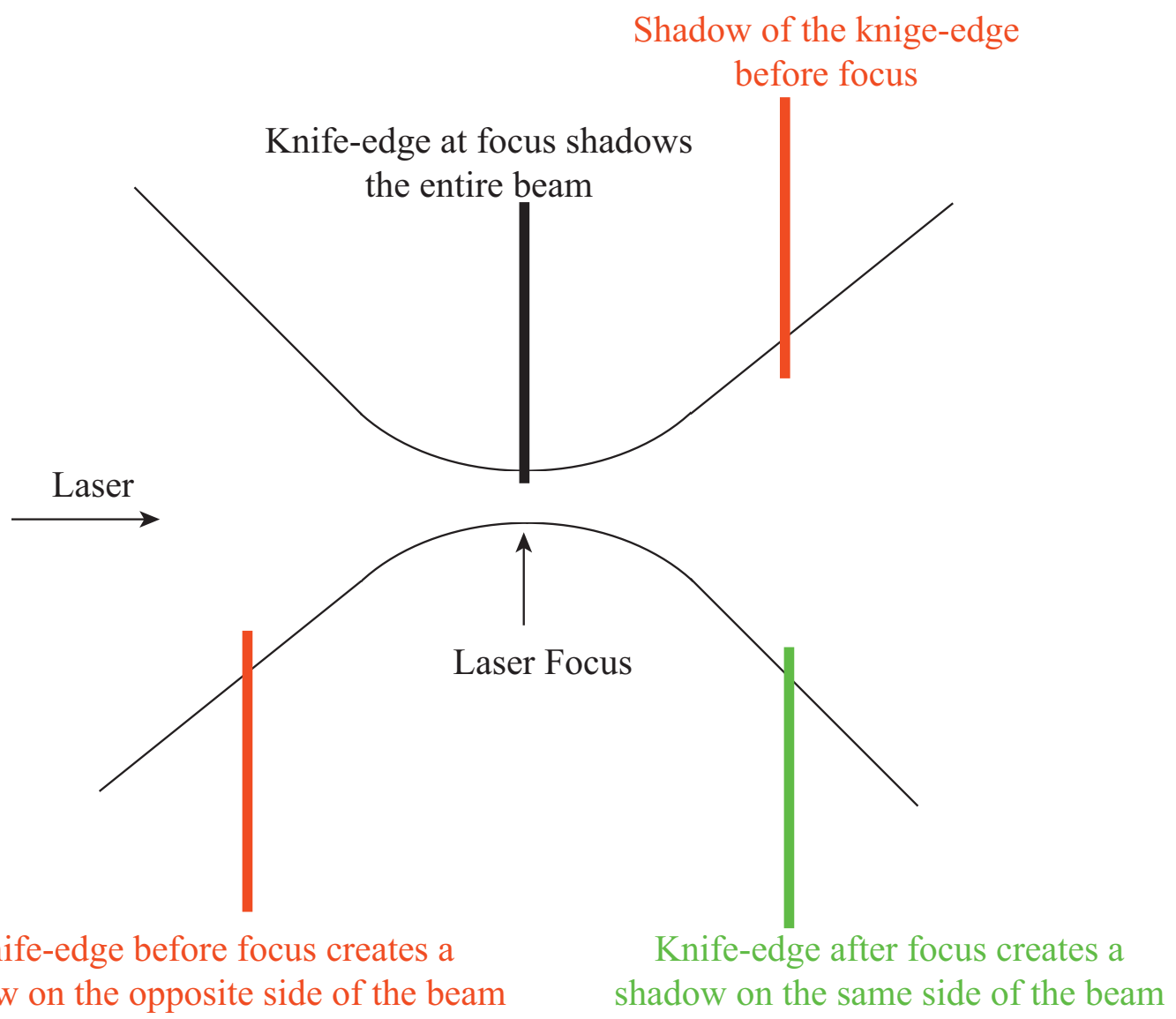

Figure 5.2: The front aperture of the gas cell is used as a knife-edge to align the gas cell. 
was not possible over the corresponding $>1 \mathrm{~cm}$ distances required for dephasing. To overcome this limitation a new target platform has been developed, where a gas cell replaces the gas jet targets. A photograph of the gas cell is shown in Fig. 5.1.

As with the gas jets, interferometry is performed in the transverse direction from the laser propagation axis. Here the optical axis is available due to the gas cell being constructed out of polycarbonate. The region of the cell that is filled with gas is $6 \times 6 \times 14 \mathrm{~mm}$, and the intereferometer sees the central $9 \mathrm{~mm}$ of the plasma along the laser axis. Off-line measurements of the gas fill time at the center of the cell have been performed with Ar using time-resolved interferometry in an offset-Sagnac configuration and indicate that the pressure inside the cell reaches steady-state after $50 \mathrm{~ms}$. Measurements taken during high-power shots during the experiment are consistent with the profile shown in Fig. 3.6, but at densities as low as $1.3 \times 10^{18} \mathrm{~cm}^{-3}$.

The alignment technique for the gas cell differs from that used with the gas jets. Rather than looking for the center of the lobe emission pattern from ionizing low pressure air, the front aperture of the gas cell is used as a knife-edge to shadow the beam as shown in Fig. 5.2. Within one Rayleigh length ahead of or behind focus, moving the knife-edge into the beam causes the transmitted beam to become shadowed just as if it were placed at focus. The determination of the best focus is made by measuring the length over which this uniform shadowing occurs and setting the plane of the aperture at the center of that range. The transverse axes are aligned by moving the gas cell up/down and left/right to observe where the transmitted beam is blocked by the aperture, and placing the cell such that the laser is in the center of both of these measured ranges (the center of the aperture).

\subsection{Ionization-induced Injection Mechanism}

Since self-trapping does not occur at electron densities relevant for $>1 \mathrm{GeV}$ electron acceleration, a different mechanism has been developed to inject and trap electrons into low density wakes. The technique, termed ionization-induced injection[15, 16], is enabled by adding small concentrations of high-Z dopant gas 


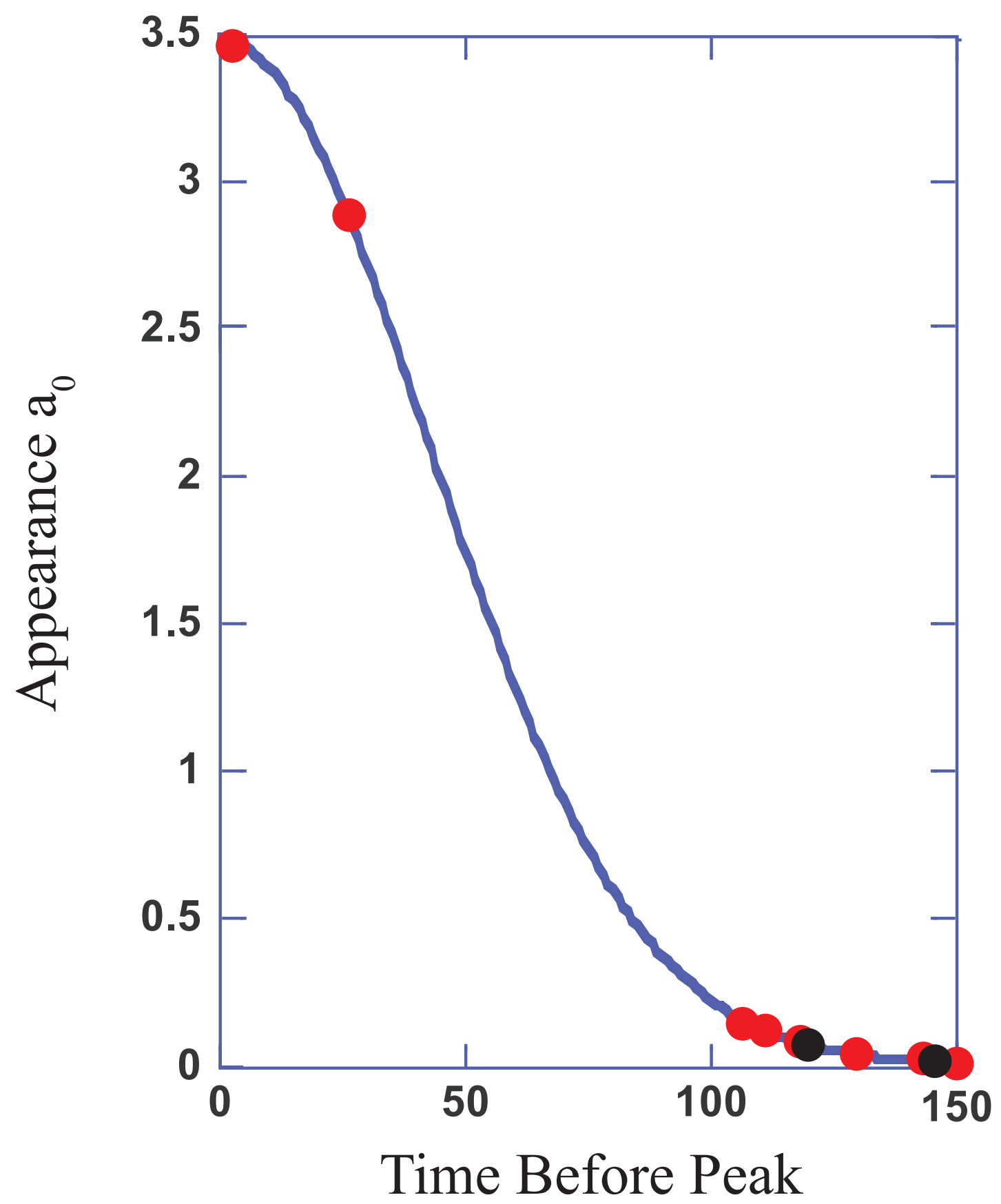

Figure 5.3: The temporal profile of a 60 fs laser pulse with a peak $\mathrm{a}_{0}=3.5$ is shown with the ionization locations for He (black circles) and $\mathrm{O}$ (red circles) electrons. 


\begin{tabular}{|l|c|r|}
\hline Ionization State & Ionization Potential $(\mathrm{eV})$ & $\mathrm{a}_{0}$ \\
\hline He I & 24.6 & 0.030 \\
He II & 54.4 & 0.072 \\
O I & 13.6 & 0.011 \\
O II & 35.1 & 0.033 \\
O III & 54.9 & 0.053 \\
O IV & 77.4 & 0.078 \\
O V & 113.9 & 0.132 \\
O VI & 138.1 & 0.162 \\
O VII & 739.3 & 2.85 \\
O VIII & 871.4 & 3.49 \\
\hline
\end{tabular}

Table 5.1: The ionization potentials and corresponding the $\mathrm{a}_{0}$ required to ionize as determined from ADK tunnel ionization[2] for He and O.

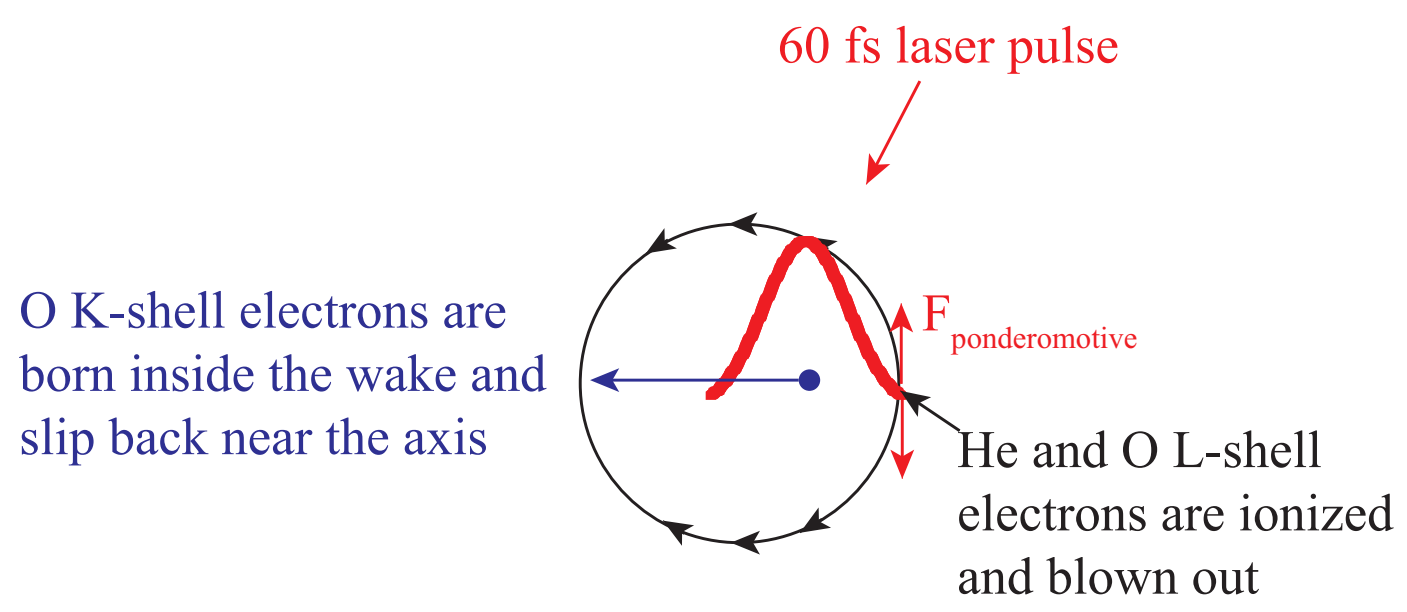

Figure 5.4: The trapping process for K-shell O electrons born inside the wake. 


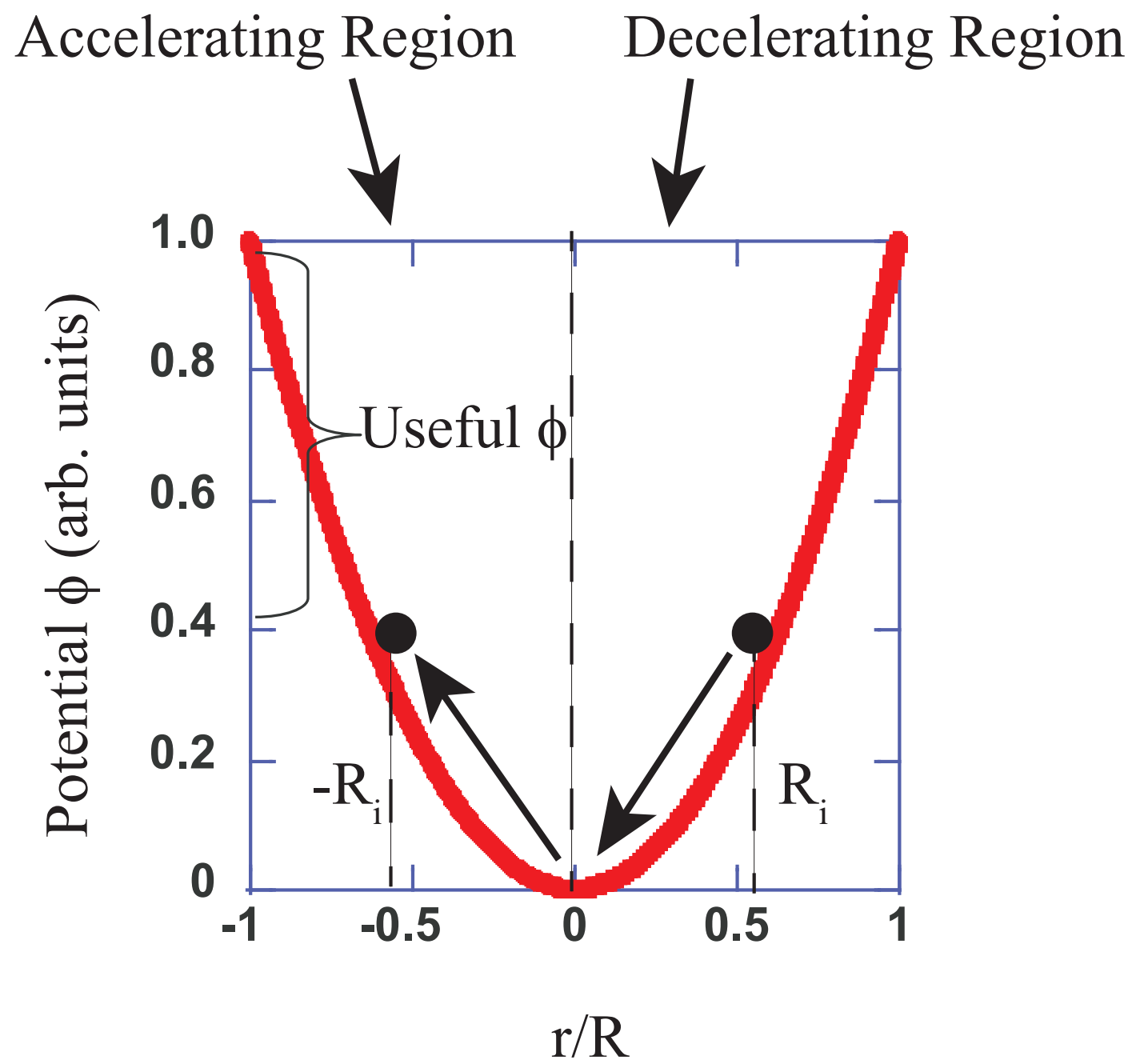

Figure 5.5: An electron ionized at a radius $\mathrm{r}=\mathrm{R}_{i}$ from the center of the wake does not start gaining energy from the wake until it reaches the position $\mathrm{r}=-\mathrm{R}_{i}$. The electron can then only become trapped if the remaining potential available is sufficient for the electron to reach the phase velocity of the wake before arriving at the back of the bucket (at radius $-\mathrm{R}$ ). 
to the nominal He gas typically used in these LWFA experiments. The choice of the dopant gas is determined by the intensity of the laser. Table 5.1 shows the ionization potentials for He and O. From ADK tunnel ionization theory[2] a corresponding laser intensity, or vector potential $\mathrm{a}_{0}$, can be calculated to ionize each state in a single cycle of the laser pulse; this required $\mathrm{a}_{0}$ from the ADK theory is also given in the table.

Fig. 5.3 shows the vector potential of the laser pulse in time on the laser axis assuming a gaussian temporal intensity profile of the form $I=I_{0} e^{-2 t^{2} / \tau^{2}}$ with $\tau=60$ fs and $\mathrm{I}_{0}=2.65 \times 10^{19} \mathrm{~W} / \mathrm{cm}^{2}$. Also shown are the locations within the temporal profile where the vector potentials corresponding to the ionization of He and $\mathrm{O}$ from Table 5.1 are achieved. The L-shell $\mathrm{O}$ electrons are ionized at nearly the same locations as the He electrons, while the K-shell O electrons are not able to be ionized until nearly the peak of the laser pulse. OSIRIS simulations with $\mathrm{He}+\mathrm{O}$ mixtures have been performed with the $\mathrm{O}$ electrons tagged as different species[13]. It is confirmed in these simulations that the L-shell O electrons follow nearly identical trajectories during the blowout as do the He electrons. The Kshell $\mathrm{O}$ electrons experience a different ponderomotive force than the rest of the electrons, and are not blown out to the radius $\mathrm{R}$ determined in $\mathrm{Ch}$. 2. Instead they slide backward in the wake from the point of ionization and are trapped if they gain enough energy from the electric field inside the wake to move at the wake phase velocity before reaching the back of the bucket. This process is shown in Fig. 5.4.

The peak laser intensity determines which gases can be candidates for the dopant. If the ionization potential for the K-shell electrons is above the peak intensity available then these electrons are never ionized and thus no electrons are trapped in the wake. Likewise, an electron born too early in the laser pulse (in the case that the peak intensity is well above the ionization potential) may not be able to gain enough energy from the wakefield to reach the wake phase velocity before slipping out of the back of the bucket. Recalling that the electric field $\mathbf{E}$ inside the wake is linear (except for the arrow spike due to the electron sheath at the back of the bucket, which is neglected here), the potential $\phi$ which satisfies 
the electrostatic $\mathbf{E}=-\nabla \phi$ is quadratic (and symmetric, as in the energy gain profile from Fig. 4.2). An electron born (at rest) in the front of the wake at a radius $\mathrm{r}=\mathrm{R}_{i}$ from the center is initially pulled backward in the wake because it is in the decelerating phase of the wakefield. Just as a ball rolling in a potential well, it continues to slip backward to a radius $\mathrm{r}=-\mathrm{R}_{i}$ before it actually begins gaining energy from the wake as shown in Fig. 5.5. It is then the remaining potential from the point $\mathrm{r}=-\mathrm{R}_{i}$ to $\mathrm{r}=-\mathrm{R}$ that is able to be imparted to the electron before it reaches the back of the bucket. From this picture it is evident that electrons born at the center of the wake have the highest probability to be trapped, as the useful potential is the full potential of the wake; likewise, electrons born at $\mathrm{r}=\mathrm{R}$ can never become trapped due to the symmetry of the potential. This theory is very well developed in the work of Pak et. al.[15].

In summary, ionization-induced injection is enabled by adding few-percent concentrations of high-Z gas to the background He. Electrons which are ionized in the appropriate phase of the wake can gain sufficient energy from the wake to become trapped before being lost at the back of the bucket. The choice of dopant gas is then a function of the available laser intensity, as this determines which gases can be ionized and where in the laser pulse (wake) ionization (injection) occurs.

\subsection{Experimental Results}

Experiments using the Callisto Laser System have been performed with the gas cell target in the same experimental configuration as the gas jet experiments of the previous chapter. However, improvements to the laser focusing resulted in $50 \%$ coupling of the laser power into the $1 / \mathrm{e}^{2}$ radius spot (compared to $30 \%$ in the gas jet experiments). Fig. 5.6 shows the result of a $\mathrm{P}=110 \mathrm{TW}$ (coupled) experiment with an electron density of $1.3 \times 10^{18} \mathrm{~cm}^{-3}$ in the $1.4 \mathrm{~cm}$-long gas cell. The peak electron energy determined by the two-screen spectrometer system is $1.45 \pm 0.1 \mathrm{GeV}$, the highest energy electrons ever produced from laser wakefield acceleration[13]. Additionally, the forward optical diagnostics indicate that the laser pulse was self-guided over the entire length of the gas cell, and that 

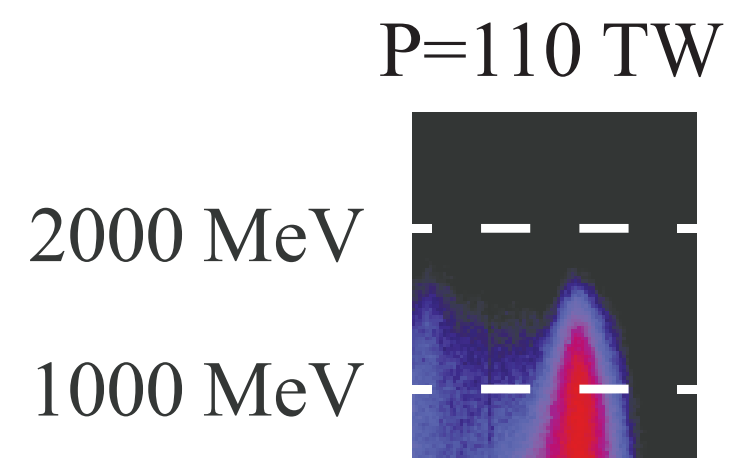

$500 \mathrm{MeV}$

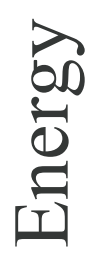

总

$100 \mathrm{MeV}$
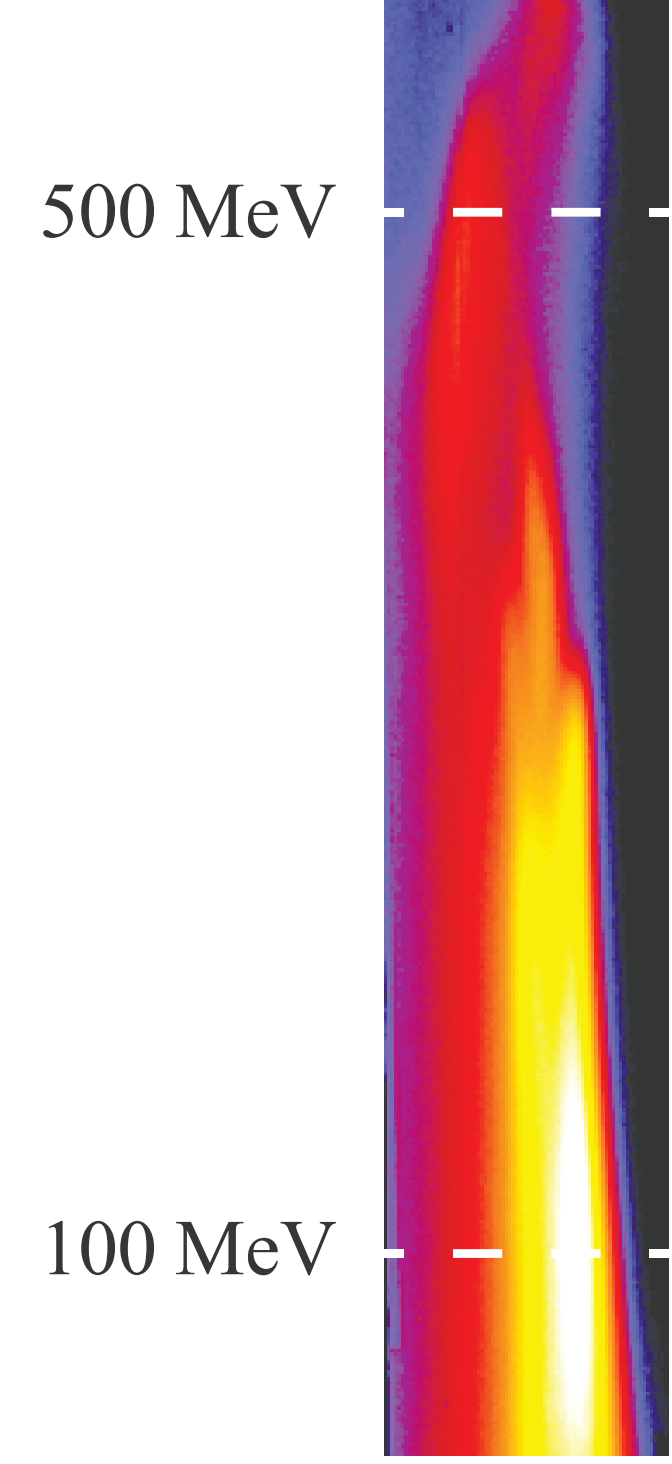

\section{$1.3 \times 10^{18} \mathrm{~cm}^{-3}$ Electron Density}

Figure 5.6: Electron spectrum from a $\mathrm{P}=110 \mathrm{TW}$ experiment with an electron density of $1.3 \times 10^{18} \mathrm{~cm}^{-3}$. The peak energy is $1.45 \mathrm{GeV}$, but the energy spread is quasi-continuous due to the method of injection. 
a strong wake was continuously driven[13]. The dephasing-limited energy gain for this power and density is $1.47 \mathrm{GeV}$, with a dephasing length of $1.35 \mathrm{~cm}$. This also implies that the wake must have existed throughout the entire gas cell in order for electrons to have achieved $99 \%$ of the maximum possible energy gain.

To achieve this result the gas composition in the cell was changed from pure He to $97 \% \mathrm{He}$ and $3 \% \mathrm{CO}_{2}$ by partial pressure in order to enable ionization-induced injection. At the same density with pure He no electrons were observed on the image plates. The choice of $\mathrm{CO}_{2}$ as the dopant gas was to account for shot-to-shot fluctuations in the laser energy (as the C K-shell electrons are easier to ionize than those of $\mathrm{O}$ ). For the $110 \mathrm{TW}$ shot the peak $\mathrm{a}_{0}=3.8$, which is just slightly above the required $\mathrm{a}_{0}$ to ionize the last state of $\mathrm{O}$ according to the ADK theory. OSIRIS simulations of the tunnel ionization process for the conditions of this shot indicate that only $\mathrm{O}^{7}$ and $\mathrm{O}^{8}$ electrons are trapped into the wake; the $\mathrm{C}^{5}$ and $\mathrm{C}^{6}$ electrons were born in the wrong phase to be trapped at this laser power, while all of the L-shell electrons from both $\mathrm{C}$ and $\mathrm{O}$ were blown out with the He electrons.

The parameters of this experiment were chosen to match the dephasing length to the length of the gas cell and provide the maximum possible electron energy gain. While utilizing ionization-induced injection did successfully allow electrons to be trapped at the correct density, there is an unfortunate consequence to this method of injection. The energy spread of the electron beam in Fig. ?? is nearly continuous; electrons have all energies from $\sim 100 \mathrm{MeV}$ to $1.45 \mathrm{GeV}$. This is a direct consequence of the ionization-induced injection scheme. O K-shell electrons are born in regions of high laser intensity. As the laser pulse propagates at its self-guided spot size, the intensity remains high enough to not only drive the wake over the entire length of the gas cell but also to continuously ionize the $\mathrm{K}$-shell of O. As a result charge is continuously trapped into the wake, filling the entire range of electron energies. This behavior is also observed in the OSIRIS simulations[13].

Up to this point the physics of laser wakefield acceleration has been shown to be in excellent agreement with the phenomenological theory put forth by Lu et. al. The previous chapter showed that by decreasing electron density and 
increasing plasma length the electron beam energy increased in accordance with Eq. 2.26. During these experiments a threshold for the self-trapping mechanism was discovered, which limited electron energies to less than $1 \mathrm{GeV}$. This chapter described an experiment involving ionization-induced injection, which allowed for low density trapping and $1.45 \mathrm{GeV}$ electron energy production. However, the new trapping scheme leads to quasi-continuous electron energy spread, which is undesirable in the particle acceleration community. If electron beams produced from LWFA are ever to be comparable to those produced in conventional RF linear accelerators the energy spread must be reduced to the percent level. The next chapter describes a modification to the gas cell target platform that achieves this goal, generating a $0.46 \mathrm{GeV}$ electron beam with $5 \%$ energy spread using the ionization-induced injection mechanism[17]. 


\section{Chapter 6}

\section{Electron Beam Energy Spread Reduction by Limiting the Ionization-Induced Injection Region}

It is evident from the LWFA scaling laws and the data presented in the previous chapters that in order to access electron energies exceeding $1 \mathrm{GeV}$ with conventional laser systems $(<100 \mathrm{TW})$ electron densities approaching $\sim 10^{18} \mathrm{~cm}^{-3}$ are required. Previous studies have shown that electron self-trapping is not an effective injection mechanism at these densities [14]. Ionization induced injection $[15,16]$, which is enabled by adding percent level concentrations of high-Z dopant gas to the low-Z background, has enabled trapping at electron densities as low as $1.3 \times 10^{18} \mathrm{~cm}^{-3}$ and peak electron energies of $\sim 1.5 \mathrm{GeV}$ from a centimeterscale plasma [13]. However, the electron energy spectrum was very broad due to continuous injection of electrons into the wake.

This chapter is concerned with reducing the energy spread of a high energy beam by separation of the injection and acceleration processes, and has resulted in the development of a two-stage LWFA. Charge is initially injected over a limited distance via ionization induced injection in a He- $\mathrm{N}_{2}$ mixture, and is then further 
accelerated in a pure He region without broadening the energy spectrum. Matching of electron density in these independent stages is critical for generating high quality, high energy electron beams. The consequences of a non-uniform density along the interaction length are examined by considering a density step at the interface between the stages. It will be shown that stepping down in density reduces the overall energy gain of the system, while stepping up can provide enhanced energy gain. However, in the latter case charge must be accelerated a minimum distance before the step to remain in the first wake period after the interface, and some charge will always be lost.

\subsection{Consequences of non-uniformities in the lon- gitudinal density profile}

Consider an electron trapped at the rear of the ion cavity (one radius $\mathrm{R}$ from the center of the cavity). The electron slips forward in the cavity as it accelerates, and reaches the center of the cavity after one dephasing length $\mathrm{L}$ in the lab frame. If during this period there is an abrupt change in the electron density, which will be represented as a density step for this discussion, the radius of the ion cavity will change to the size corresponding to the density after the step. This process is assumed to be sufficiently fast (on the order of the pulse duration) to be instantaneous for the electrons residing inside the cavity. Since the cavity forms from front to back, the center of the cavity shifts relative to the electrons, modifying the remaining useful accelerating region of the interaction.

We first examine the case of a step down in density, illustrated in Figure 6.1a, where electrons have been accelerated over the distance z1 in the lab frame (corresponding to a distance of R1-b in the cavity). The size of the cavity increases after the step, and the cavity center moves away from the laser pulse by (R2-R1). The remaining accelerating region in the cavity is shorter than b, so electrons will dephase after a shorter distance than the initial dephasing length. Additionally, the longitudinal electric field in the lower density region is reduced from that of the high density region. These two effects together act to reduce 
a)
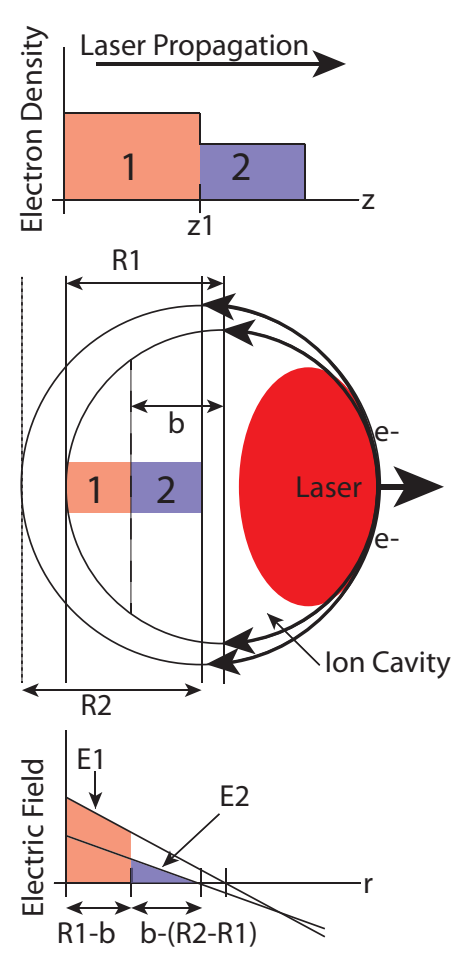

b)
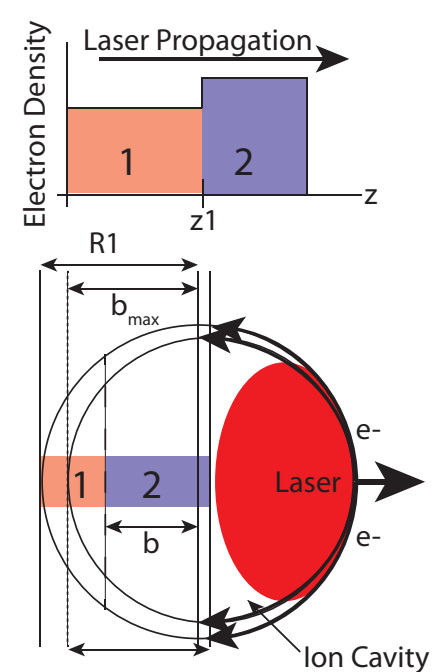

$\mathrm{R} 2$

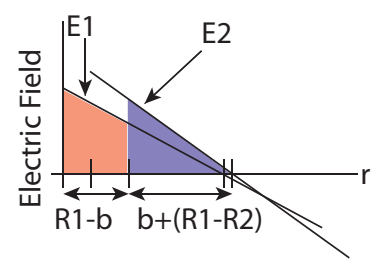

Figure 6.1: Schematic representation of the effects of a density step in a LWFA gas target. The laser propagates a distance $\mathrm{z} 1$ in the target at density $\mathrm{n} 1$, forming a blown-out ion cavity with radius R1. Electrons trapped at the rear of the cavity (and at the beginning of the target) have traveled a distance R1-b in the cavity when the laser reaches $\mathrm{z} 1$ in the target (the case $\mathrm{z} 1=\mathrm{L}$ corresponds to electrons reaching the cavity center, where $b=0$, and dephasing). a) The density is reduced at $\mathrm{z} 1$, causing the cavity radius to increase to $\mathrm{R} 2$ and the cavity center to shift toward the electrons. b) The density increases at $\mathrm{z} 1$, causing the blow-out radius to decrease to R2 and the cavity center to shift away from the electrons. 
the maximum energy gain achievable in the system. Furthermore, electrons that gained the maximum possible energy and dephased in the first region $(b=0)$ now reside past the center of the cavity, where the electric field decelerates electrons.

Conversely, if the density increases after the propagation distance z1, the cavity radius decreases. Figure $6.1 \mathrm{~b}$ shows that under these conditions the cavity center slips toward the laser pulse, lengthening the remaining accelerating region from $b$ to $b+(R 1-R 2)$. Since the density is higher in this region the electric field is increased, and these effects now increase the maximum possible energy gain. Even electrons that have reached dephasing at the time the density step occurs can see an increased energy gain. Note the distance bmax $=2(\mathrm{R} 1-\mathrm{R} 2)$ in Figure $6.1 \mathrm{~b}$; this is the maximum cavity region that can remain before the step to prevent charge from residing outside of the first wake period after the density transition. The criterion placed on the propagation distance is then $\mathrm{z} 1 \geq \mathrm{L} 1(1-(\mathrm{bmax} / \mathrm{R} 1))$. If the step occurs before this point, the rear of the high density cavity will form ahead of the electrons; this will cause the electrons to reside in the decelerating field portion of the next period of the wake.

In a two-stage LWFA experiment, should a density step occur it would be at the interface between the stages. If the first stage (the injector) is filled to sufficiently high density He for self-trapping to occur, then a transition to lower density He in the second stage (the accelerator) could be used to terminate the injection. While this may produce a narrower energy spectrum than a constant density He experiment, it comes at the expense of peak energy. However, taking advantage of ionization induced injection in the first stage would allow for trapping at low density, where the density in the accelerator can then be increased to improve energy gain. In order to maintain a limited energy spread, an upper limit on the accelerator density would be the self-trapping threshold of He. There will now be a trade-off between energy spread and total charge, as only the electrons that travel the required length in the injector will remain in the first wake period after the transition. For injector densities between $2-3 \times 10^{18} \mathrm{~cm}^{-3}$ and an accelerator density of $4 \times 10^{18} \mathrm{~cm}^{-3}$, the benefit to attempting the density increase is not more than a $5 \%$ increase in peak energy to preserve all charge within $10 \%$ of 
the peak energy. Therefore, our experiment attempts to equalize the densities in

the injector and accelerator until more reliable trapping at densities below $1 \times 10^{18}$ $\mathrm{cm}^{-3}$ is demonstrated and significant benefit from a higher density accelerator can be realized.

\subsection{Two-stage LWFA experiment}

In this chapter, we report that by limiting ionization-induced injection to a distinct region, a $460 \mathrm{MeV}$ electron beam with <5\% energy spread is produced in a two-stage LWFA. The experiments are performed with an $8 \mathrm{~mm}$ long, twostage gas cell, shown schematically in Figure 6.2. The cell is comprised of a 3 mm injection stage, filled with a mixture of $99.5 \% \mathrm{He}$ and $0.5 \% \mathrm{~N}_{2}$ gas, separated by a $1 \mathrm{~mm}$ diameter aperture from an immediately adjacent $5 \mathrm{~mm}$ acceleration stage containing pure He. This result is obtained at an electron density where self-trapping in He is not observed, and spectroscopic measurements of the gas species along the cell indicate that the $\mathrm{N}_{2}$ is confined to the injector stage. The spatial and spectral content of the laser beam at the exit of the gas cell imply that the laser is both self-guided and drives a wake over the entire length of the injector and accelerator stages.

These studies are conducted at the Jupiter Laser Facility, Lawrence Livermore National Laboratory, using the Callisto laser system. The laser beam, which delivers up to $200 \mathrm{TW}$ of power in a 60 fs laser pulse, is focused with an $\mathrm{f} / 8$, off-axis parabolic mirror to a vacuum spot size $\mathrm{w}_{0}$ of $15 \mu \mathrm{m}$ at the $1 / \mathrm{e}^{2}$ intensity point (containing $30 \%$ of the laser power) at a position $750 \mu \mathrm{m}$ inside the gas cell. For coupled laser powers of 30-60 TW $\mathrm{a}_{0}=2-2.8$, while the ionization thresholds to produce $\mathrm{N}^{6+}$ and $\mathrm{N}^{7+}$ are 1.8 and 2.3, respectively. Therefore, K-shell electrons from the nitrogen gas in the injector stage will be continuously ionized near the peak of the laser pulse, which resides near the zero-crossing of the longitudinal electric field. This injection phase is near-optimum for trapping because these electrons can now experience the entire potential difference within the wake [15].

Figure 6.3a shows electron beam data on the first of two image plates from 


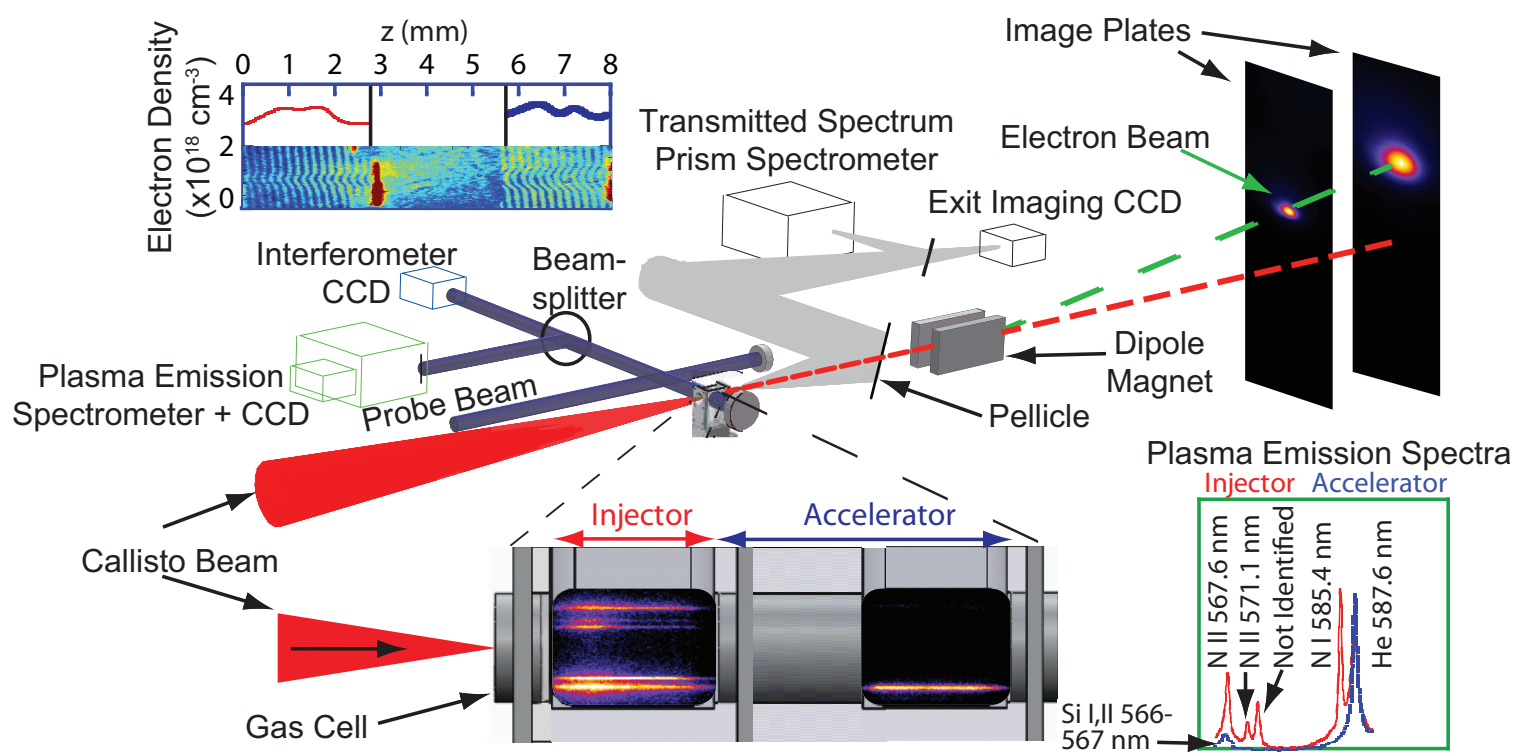

Figure 6.2: Schematic of the experimental setup showing the $800 \mathrm{~nm}$ Callisto laser beam (in red), the two-stage gas cell, the $800 \mathrm{~nm}$ probe beam (in blue), a measured interferogram with its associated Abel inverted density profile (above the interferometer CCD camera), the plasma emission spectrometer and CCD camera, plasma emission images (shown inside the gas cell windows) with the spatially resolved plasma emission spectrum along the laser axis from each stage of the gas cell, the vacuum laser axis after the gas cell (red dashed line), the 0.42T dipole magnet $(20 \mathrm{~cm}$ long, centered $66 \mathrm{~cm}$ from the exit of the gas cell), the deflected electron trajectory (green dashed line) onto the image plates (located $132 \mathrm{~cm}$ and $192 \mathrm{~cm}$ from the exit of the gas cell), and the optical path of the transmitted laser light (in grey) to an imaging system and a prism spectrometer. The transverse size of the plasma observed in the interferogram is larger in the accelerator section because the non-coupled (and therefore unguided) diffracting laser light ionizes a volume larger than the sub-50 $\mu \mathrm{m}$ wake, which is not resolved by this diagnostic. Optical access for the transverse diagnostics is provided by constructing the walls of the gas cell from microscope slides pressed against rubber gaskets to form a seal. The source of the Si line in the plasma emission image is suspected to be minute amounts of Si out-gassing from the gaskets under vacuum. The gas cell entrance (exit) aperture is $0.5(2.0) \mathrm{mm}$. 

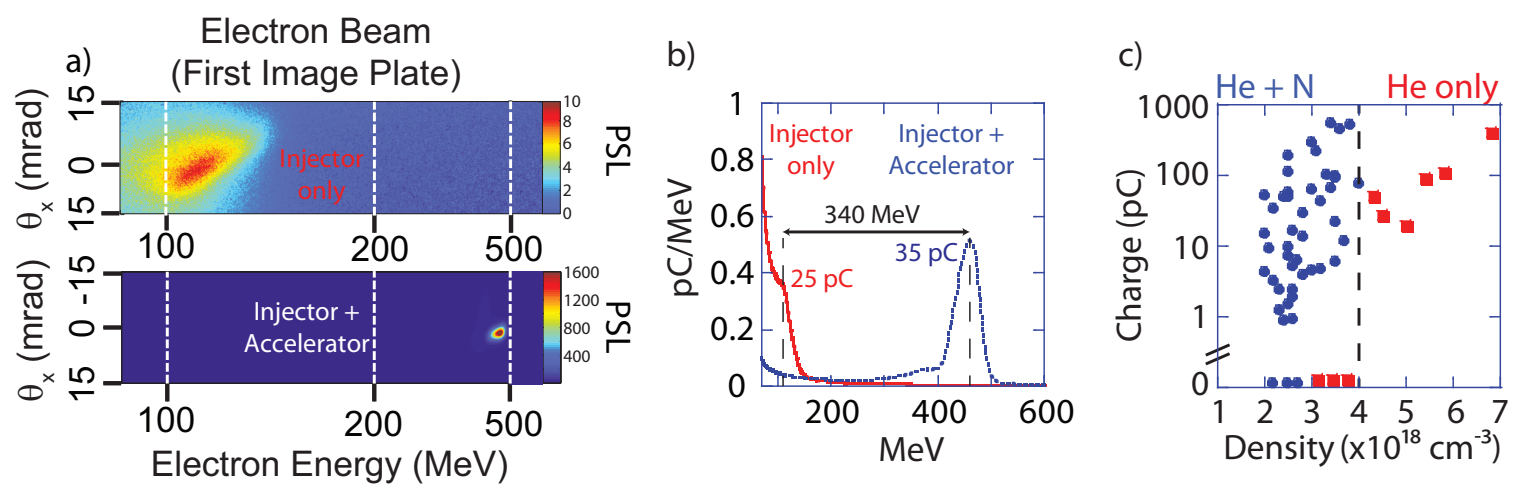

Figure 6.3: a) Magnetically dispersed electron beam images from a $4 \mathrm{~mm}$ injector only gas cell (top) and the $8 \mathrm{~mm}$ two-stage cell (bottom). b) Electron spectra above $70 \mathrm{MeV}$ for: the $8 \mathrm{~mm}$ two-stage injector-accelerator cell (dotted blue curve) filled to an electron density of $3 \times 10^{18} \mathrm{~cm}^{-3}$ in each stage for a coupled laser power of $40 \mathrm{TW}$; the $4 \mathrm{~mm}$ injector-only cell (solid red curve) filled to an electron density of $3.4 \times 10^{18} \mathrm{~cm}^{-3}$ for a coupled laser power of $50 \mathrm{TW}$. The injector gas fill in each case is $99.5 \% \mathrm{He}$ and $0.5 \% \mathrm{~N}_{2}$, and the total charge is indicated for each spectrum. c) The total observed charge above $70 \mathrm{MeV}$ for injector gas fills of pure He (red squares) and $99.5 \%$ He with $0.5 \% \mathrm{~N}_{2}$ (blue circles) for coupled laser powers between 30-60 TW.

injector only and injector + accelerator experiments. The electrons are dispersed by the dipole magnet and are recorded on two image plates to determine independently their energy and exit angle upon leaving the plasma [37, 3]. The corresponding spectra are shown in Figure 6.3b, where a 460 $\pm 25 \mathrm{MeV}$ electron beam containing $\sim 35 \mathrm{pC}$ of charge is produced in the two-stage cell with a density of $3 \pm 0.3 \times 10^{18} \mathrm{~cm}^{-3}$ (see Figure 6.2; the standard deviation of the longitudinal density profile is $5.6 \%$ ) for a coupled laser power of $40 \mathrm{TW}$. The injector-only spectrum is broad, consistent with ionization-induced injection [15], and exhibits a slight peak at $120 \mathrm{MeV}$. Conversely, after deconvolution in quadrature of the 2.3 mrad transverse beam size with the spectrum of Fig. 6.3b, the energy spread $\Delta \mathrm{E}$ of the two-stage experiment is inferred to be $5 \%$.

A Mach-Zehnder interferometer allows the electron density to be measured along the gas cell with a spatial resolution of $125 \mu \mathrm{m}$, and is timed such that the 100 fs probe beam traverses the plasma $\sim 20$ ps after the main beam has exited the gas cell. While injector-only data was taken for densities as low as $2 \times 10^{18} \mathrm{~cm}^{-3}$, 
the minimum density where the injector and accelerator densities were matched was $3 \times 10^{18} \mathrm{~cm}^{-3}$. Due to the additional electrons from the fully-ionized nitrogen atoms in the injector, the neutral gas pressure in the accelerator must be slightly higher than in the injector in order to balance the electron density between the two stages. This results in a small upstream pressure which helps to confine the nitrogen to the injector. The gas species in each stage is determined by 1:1 imaging the plasma emission along the laser propagation axis onto the $50 \mu \mathrm{m}$ entrance slit of a $1 / 3-m$ spectrometer coupled to a 16-bit charge-coupled device (CCD) camera, where the spectral resolution of the system is $2.5 \AA$. As illustrated by the plasma emission lines in Figure 6.2, which correspond to the same experiment shown in the interferogram, nitrogen is present in the injector stage only. As shown in Figure 6.3c, no self-injected electrons are observed in pure He plasma for electron densities below $4 \times 10^{18} \mathrm{~cm}^{-3}$ (at coupled laser powers $\leq 60 \mathrm{TW}$ ). This, along with the absence of nitrogen lines in the accelerator stage, indicates that the observed electrons are from the nitrogen dopant gas in the injector.

Figure 6.4 shows the spatial and spectral transmitted laser light properties for the injector-only and the two-stage gas cell, which demonstrate self-guiding and wakefield excitation in the self-guided blowout regime. Most of the transmitted light is confined to $100-130 \mu \mathrm{m}$ FWHM spots, which is much smaller than the unguided, vacuum spot sizes at those planes, and indicates the laser pulse was self-guided in both cases. Figures 6.4(c) and (d) show the respective "open-slit" spectra of the transmitted light, where the spectral features are dominated by the guided, bright features of Figs. 6.4(a) and (b). The spectrum corresponding to the injector-only case shows the expected blue- and red-shifts arising from photon acceleration/ionization [38] and local pump depletion [31], respectively, experienced by portions of the incident laser pulse as it produces the plasma and excites the wake within the injector stage. In the longer, two-stage case, the extent of the red-shifting approximately doubles compared with the injector-only data, indicating that, in addition to the laser pulse continuing to self-guide across the interface between the two stages, the wake is also driven over the extended distance.

Three dimensional particle-in-cell simulations performed using the mas- 


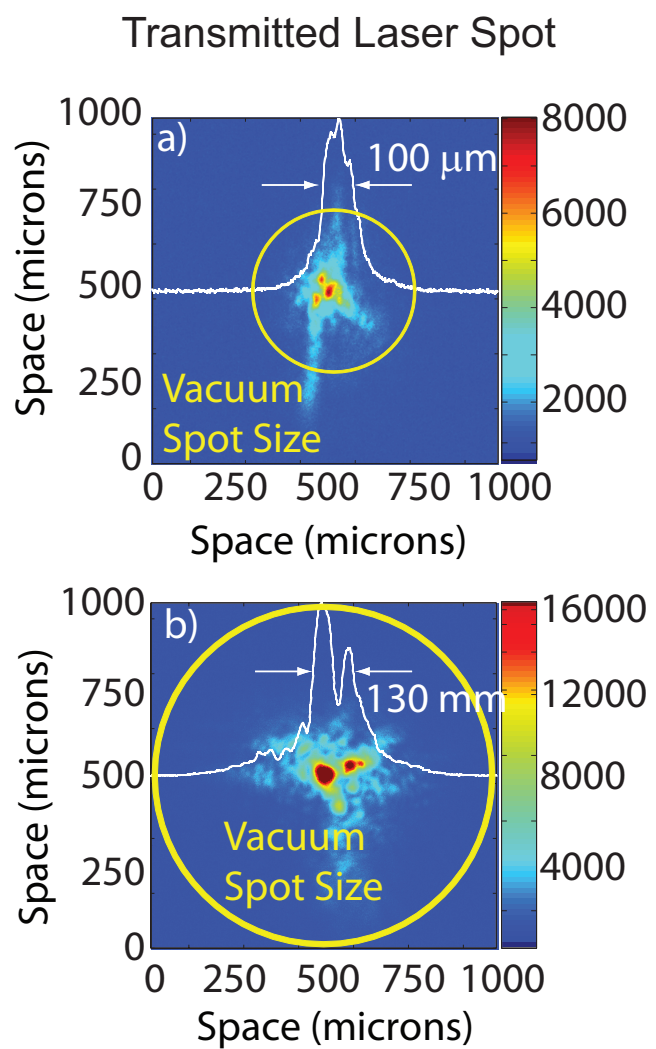

\section{Transmitted Laser Spectrum}
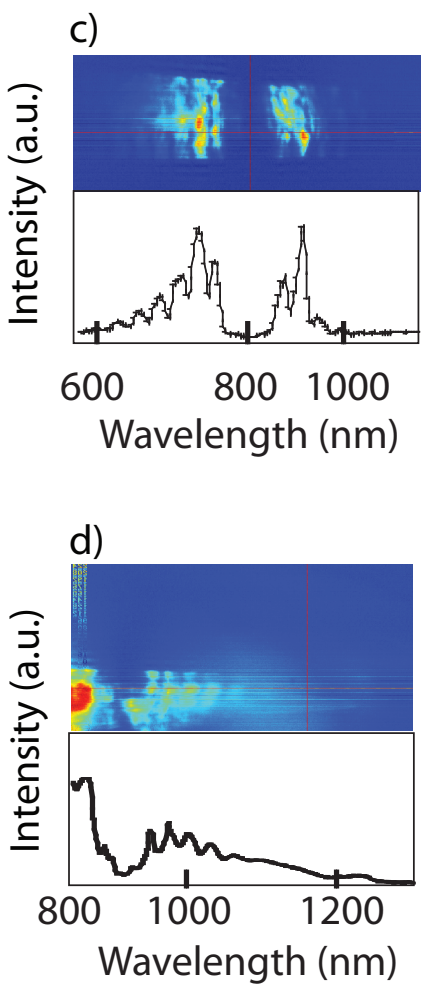

Figure 6.4: a,b) Images of the transmitted laser light at the exit of the $4 \mathrm{~mm}$ injector stage and the $8 \mathrm{~mm}$ two-stage gas cell, respectively. Vertically integrated lineouts indicate the guided laser spot size, while the vacuum laser spot size at the exit of the cell is denoted for each case by a yellow circle. A long-pass filter is placed in front of the camera to attenuate unshifted and blue-shifted light. c,d) The transmitted laser spectrum from the injector only and the two-stage cell, respectively. A mask is used to block the fundamental $800 \mathrm{~nm}$ light to take better advantage of the dynamic range of the 8-bit, frame grabbed, infrared camera. The spectral fringes are due to the etalon effect of the uncoated pellicle beamsplitter $\left(5 \mu \mathrm{m}\right.$ thick at $\left.45^{\circ}\right)$ seen in Fig.6.2. For each spectrum the recorded false-color image is shown. 
sively parallel code OSIRIS [28] - utilizing a moving window and an ADK [2] ionization model - demonstrate the essential features of this injector-accelerator concept. The simulations were initialized with the nominal experimental parameters, where the $40 \mathrm{TW}$ laser beam was focused to a Gaussian diffraction limited spot size of 15 microns at the top of a plasma density ramp followed by a uniform $8 \mathrm{~mm}$ long ( $3 \mathrm{~mm} \mathrm{He} / \mathrm{N}_{2}$ gas, $5 \mathrm{~mm}$ He Only) $3.0 \times 10^{18} \mathrm{~cm}^{-3}$ plasma. These simulations used a 130 um x 180 um x 180 um computational window corresponding to $4000 \times 300 \times 300$ grid points.

The simulation tracks the 6-D phase space of the He, N L-shell, and $\mathrm{N}$ $\mathrm{K}$-shell electrons. In the injector portion of the simulation the trapping is mainly of the N K-shell electrons, which were ionized close to the peak of the laser pulse. Although some He electrons are trapped towards the end of the accelerator section, the final energy spectrum is predominantly comprised of the $\mathrm{N}$ K-shell that form a $510 \mathrm{MeV}$ peak with a $\pm 20 \mathrm{MeV}$ energy spread. Both beam loading and phase space dynamics of the electrons are important in narrowing the eventual energy spread, and the details of this simulation will be published elsewhere. As has been reported previously $[13,14]$, the injected charge in the present simulation is significantly greater than what is observed in the experiments presented here. This is likely a result of the non-ideal initial laser conditions present in the experiment (e.g. pulse front tilt [39], non-Gaussian laser spot, radial group delay).

In conclusion, a cm-scale, two-stage injector-accelerator LWFA is shown to generate $\sim 0.5 \mathrm{GeV}$ electron beams with $<5 \%$ energy spread containing $35 \mathrm{pC}$ of charge using the ionization-induced injection mechanism. Extending the present work to densities approaching $1 \times 10^{17} \mathrm{~cm}^{-3}$ could provide a compact platform for producing high-quality, $10 \mathrm{GeV}$ electron beams with PW-class lasers for advanced light source and collider applications [40].

Chapter 6, in part, is a reprint of the material as it appears in: B.B. Pollock, G.R. Tynan, F. Albert, C. Filip, S.H. Glenzer, J. Meinecke, A. Pak, J.E. Ralph, C.E. Clayton, C. Joshi, K.A. Marsh, J. Shaw, K.L. Herpoldt, and D.H. Froula. The effects of a density mismatch in a two-stage LWFA. Proceedings of the 2011 Paricle Accelerator Conference, New York, 2011 (http://www.c- 
ad.bnl.gov/pac2011/proceedings/papers/weobs3.pdf), p. 1. The dissertation author was the primary investigator and author of this paper.

Chapter 6, in part, is a reprint of the material as it appears in: B. B. Pollock, C.E. Clayton, J.E. Ralph, F. Albert, A. Davidson, L. Divol, C. Filip, S.H. Glenzer, K. Herpoldt, W. Lu, K. Marsh, J. Meinecke, W.B. Mori, A. Pak, T.C. Rensink, J.S. Ross, J. Shaw, G.R. Tynan, C. Joshi, and D.H. Froula. Demonstration of a narrow energy spread, $0.5 \mathrm{GeV}$ electron beam from a two-stage Laser Wakefield Accelerator. Phys. Rev. Lett., 107, 045001 (2011). The dissertation author was the primary investigator and author of this paper. 


\section{Chapter 7}

\section{Conclusions}

Laser wakefield acceleration holds great promise for the future of particle acceleration. This thesis described a series of experiments which investigated the physics of LWFA in the self-guided blowout regime. Beginning with high density gas jet experiments the scaling of the electron beam energy with plasma electron density was found to be in excellent agreement with both phenomenological theory and with 3-D PIC simulations. It was also determined that self-trapping of background electrons exhibits a threshold as a function of the electron density, and at the densities required to produce electron beams with energies exceeding $1 \mathrm{GeV}$ a different mechanism was required to trap charge into low density wakes.

By introducing small concentrations of high- $Z$ gas to the nominal He background the ionization-induced injection mechanism enabled electron trapping at a density of $1.3 \times 10^{18} \mathrm{~cm}^{-3}$ and $1.45 \mathrm{GeV}$ electrons were demonstrated for the first time. This is currently the highest electron energy ever produced from LWFA. The ionization-induced trapping mechanism was also shown to generate quasicontinuous electron beam energies, which is undesirable for accelerator applications. By limiting the region over which ionization-induced trapping occurs the energy spread of the electron beams can be controlled. The development of a novel two-stage gas cell target provided the capability to tailor the gas composition in the longitudinal direction, and confine the trapping process to occur only in a limited, defined region. Using this technique a $460 \mathrm{MeV}$ electron beam was produced with an energy spread of $5 \%$. 


\subsection{Future Work}

Future experiments using the two-stage gas cell at the Callisto Laser System will pursue an increase in the electron beam energy to more than $2 \mathrm{GeV}$ with an energy spread of less than $1 \%$. This technique is directly scalable to $10 \mathrm{GeV}$ electron production, but requires more than $250 \mathrm{TW}$ of power coupled into the laser spot (which on Callisto requires 0.5-0.75 PW of compressed laser power). By varying the type and concentration of the dopant gas with the current system experiments examining laser self-guiding in high-Z gas can be performed. Additionally, ionization-induced injection experiments can be performed to maximize the total charge in the electron beams, rather than simply maximizing the energy gain. As the energy gain and charge of LWFA electrons beams increase, and the emittance and energy spread decrease, this field moves continually closer to providing the same high quality electron beam sources once available only at facilities such as SLAC at a size and cost to be practical for university, medical, and commercial applications. 


\section{Bibliography}

[1] W. Lu, M. Tzoufras, C. Joshi, F. S. Tsung, W. B. Mori, J. Vieira, R. A. Fonseca, and L. O. Silva, "Generating multi-gev electron bunches using single stage laser wakefield acceleration in a $3 \mathrm{~d}$ nonlinear regime," Phys. Rev. ST Accel. Beams, vol. 10, p. 061301, Jun 2007.

[2] M. Ammosov, N. Delone, and V. Krainov, "Tunnel ionization of complex atoms and of atomic ions in an alternating electromagnetic field," Soviet Physics - JETP, vol. 91, pp. 1191-4, Dec 1986.

[3] I. Blumenfeld, C. Clayton, F. Decker, M. Hogan, C. Huang, R. Ischebeck, R. Iverson, C. Joshi, T. Katsouleas, N. Kirby, et al., "Energy doubling of 42 gev electrons in a metre-scale plasma wakefield accelerator," Nature, vol. 445, no. 7129 , pp. 741-744, 2007.

[4] T. Tajima and J. M. Dawson, "Laser electron accelerator," Phys. Rev. Lett., vol. 43, pp. 267-270, Jul 1979.

[5] C. Joshi, W. B. Mori, T. Katsouleas, J. M. Dawson, J. M. Kindel, and D. W. Forslund, "Ultrahigh Gradient Particle-Acceleration by Intense Laser-Driven Plasma-Density Waves," Nature, vol. 311, no. 5986, pp. 525-529, 1984.

[6] C. E. Clayton, C. Joshi, C. Darrow, and D. Umstadter, "Relativistic PlasmaWave Excitation by Collinear Optical Mixing," Phys. Rev. Lett., vol. 54, no. 21, pp. 2343-2346, 1985.

[7] C. E. Clayton, K. A. Marsh, A. Dyson, M. Everett, A. Lal, W. P. Leemans, R. Williams, and C. Joshi, "Ultrahigh-Gradient Acceleration of Injected Electrons by Laser-Excited Relativistic Electron-Plasma Waves," Phys. Rev. Lett., vol. 70, pp. 37-40, JAN 41993.

[8] C. Joshi, "The development of laser- and beam-driven plasma accelerators as an experimental field," Phys. Plasmas, vol. 14, MAY 2007. 48th Annual Meeting of the Division of Plasma Physics of the APS, Philadelphia, PA, JAN 30-NOV 03, 2006. 
[9] D. Strickland and G. Mourou, "Compression of Amplified Chirped Optical Pulses," Opt. Commun., vol. 56, pp. 219-221, DEC 11985.

[10] A. Modena, Z. Najmudin, A. E. Dangor, C. E. Clayton, K. A. Marsh, C. Joshi, V. Malka, C. B. Darrow, C. Danson, D. Neely, and F. N. Walsh, "Electron Acceleration from the Breaking of Relativistic Plasma-Waves," NATURE, vol. 377, pp. 606-608, OCT 191995.

[11] W. P. Leemans, B. Nagler, A. J. Gonsalves, C. Toth, K. Nakamura, C. G. R. Geddes, E. Esarey, C. B. Schroeder, and S. M. Hooker, "Gev electron beams from a centimetre-scale accelerator," Nat. Phys., vol. 2, pp. 696-699, 2006.

[12] J. E. Ralph, K. A. Marsh, A. E. Pak, W. Lu, C. E. Clayton, F. Fang, W. B. Mori, and C. Joshi, "Self-guiding of ultrashort, relativistically intense laser pulses through underdense plasmas in the blowout regime," Phys. Rev. Lett., vol. 102, p. 175003, Apr 2009.

[13] C. E. Clayton, J. E. Ralph, F. Albert, R. A. Fonseca, S. H. Glenzer, C. Joshi, W. Lu, K. A. Marsh, S. F. Martins, W. B. Mori, A. Pak, F. S. Tsung, B. B. Pollock, J. S. Ross, L. O. Silva, and D. H. Froula, "Self-guided laser wakefield acceleration beyond 1 gev using ionization-induced injection," Phys. Rev. Lett., vol. 105, p. 105003, Sep 2010.

[14] D. H. Froula, C. E. Clayton, T. Döppner, K. A. Marsh, C. P. J. Barty, L. Divol, R. A. Fonseca, S. H. Glenzer, C. Joshi, W. Lu, S. F. Martins, P. Michel, W. B. Mori, J. P. Palastro, B. B. Pollock, A. Pak, J. E. Ralph, J. S. Ross, C. W. Siders, L. O. Silva, and T. Wang, "Measurements of the critical power for self-injection of electrons in a laser wakefield accelerator," Phys. Rev. Lett., vol. 103, p. 215006, Nov 2009.

[15] A. Pak, K. A. Marsh, S. F. Martins, W. Lu, W. B. Mori, and C. Joshi, "Injection and trapping of tunnel-ionized electrons into laser-produced wakes," Phys. Rev. Lett., vol. 104, p. 025003, Jan 2010.

[16] C. McGuffey, A. G. R. Thomas, W. Schumaker, T. Matsuoka, V. Chvykov, F. J. Dollar, G. Kalintchenko, V. Yanovsky, A. Maksimchuk, K. Krushelnick, V. Y. Bychenkov, I. V. Glazyrin, and A. V. Karpeev, "Ionization induced trapping in a laser wakefield accelerator," Phys. Rev. Lett., vol. 104, p. 025004, Jan 2010.

[17] B. B. Pollock, C. E. Clayton, J. E. Ralph, F. Albert, A. Davidson, L. Divol, C. Filip, S. H. Glenzer, K. Herpoldt, W. Lu, K. A. Marsh, J. Meinecke, W. B. Mori, A. Pak, T. C. Rensink, J. S. Ross, J. Shaw, G. R. Tynan, C. Joshi, and D. H. Froula, "Demonstration of a Narrow Energy Spread, similar to $0.5 \mathrm{GeV}$ Electron Beam from a Two-Stage Laser Wakefield Accelerator," Phys. Rev. Lett., vol. 107, JUL 182011. 
[18] D. H. Froula, L. Divol, P. Davis, J. P. Palastro, P. Michel, V. Leurent, S. H. Glenzer, B. B. Pollock, and G. Tynan, "Magnetically controlled plasma waveguide for laser wakefield acceleration," Plasma Phys. Controlled Fusion, vol. 51, FEB 2009.

[19] B. B. Pollock, D. H. Froula, G. R. Tynan, L. Divol, D. Price, R. Costa, F. Yepiz, S. Fulkerson, F. Mangini, and S. H. Glenzer, "Multicentimeter long high density magnetic plasmas for optical guiding," Rev. Sci. Instr., vol. 79, OCT 2008. 17th Topical Conference on High-Temperature Plasma Diagnostics, Albuquerque, NM, 2008.

[20] D. H. Froula, J. S. Ross, B. B. Pollock, P. Davis, A. N. James, L. Divol, M. J. Edwards, A. A. Offenberger, D. Price, R. P. J. Town, G. R. Tynan, and S. H. Glenzer, "Quenching of the nonlocal electron heat transport by large external magnetic fields in a laser-produced plasma measured with imaging thomson scattering," Phys. Rev. Lett., vol. 98, MAR 302007.

[21] B. B. Pollock, D. H. Froula, P. F. Davis, J. S. Ross, S. Fulkerson, J. Bower, J. Satariano, D. Price, K. Krushelnick, and S. H. Glenzer, "High magnetic field generation for laser-plasma experiments," Rev. Sci. Instr., vol. 77, NOV 2006.

[22] B. B. Pollock, P. Davis, L. Divol, S. H. Glenzer, J. P. Palastro, D. Price, G. R. Tynan, and D. H. Froula, "Magnetically Controlled Optical Plasma Waveguide for Electron Acceleration," in ADVANCED ACCELERATOR CONCEPTS (Schroeder, CB and Leemans, W and Esarey, E, ed.), vol. 1086 of AIP Conference Proceedings, pp. 165-170, US DOE; Lawrence Berkeley Natl Lab; Univ Calif Berkeley, 2009. 13th Advanced Accelerator Concepts Workshop, Santa Cruz, CA, JUL 27-AUG 02, 2008.

[23] P. Mora and T. M. Antonsen, "Kinetic modeling of intense, short laser pulses propagating in tenuous plasmas," Phys. Plasmas, vol. 4, pp. 217-229, JAN 1997.

[24] D. J. Spence and S. M. Hooker, "Investigation of a hydrogen plasma waveguide," Phys. Rev. E, vol. 63, p. 015401, Dec 2000.

[25] F. S. Tsung, W. Lu, M. Tzoufras, W. B. Mori, C. Joshi, J. M. Vieira, L. O. Silva, and R. A. Fonseca, "Simulation of monoenergetic electron generation via laser wakefield accelerators for 5-25 tw lasers," Physics of Plasmas, vol. 13, no. 5, p. 056708, 2006.

[26] E. Esarey, P. Sprangle, J. Krall, and A. Ting, "Overview of plasma-based accelerator concepts," IEEE Trans. Plamsa Sci., vol. 24, pp. 252-288, APR 1996. International Workshop on 2nd-Generation Plasma Accelerators, KARDAMYLI, GREECE, JUN 26-30, 1995. 
[27] Guo-Zheng Sun, E. Ott, Y. Lee, and P. Guzdar, "Self-focusing of short intense pulses in plasmas," Phys. Fluids, pp. 526-32.

[28] R. Fonseca, L. Silva, F. Tsung, V. Decyk, W. Lu, C. Ren, W. Mori, S. Deng, S. Lee, T. Katsouleas, and J. Adam, "Osiris: A three-dimensional, fully relativistic particle in cell code for modeling plasma based accelerators," Lect. Notes Comp. Sci., vol. 2331, pp. 324-351, 2002.

[29] I. Kostyukov, E. Nerush, A. Pukhov, and V. Seredov, "Electron self-injection in multidimensional relativistic-plasma wake fields," Phys. Rev. Lett., vol. 103, p. 175003 , Oct 2009.

[30] W. Lu, C. Huang, M. Zhou, M. Tzoufras, F. S. Tsung, W. B. Mori, and T. Katsouleas, "A nonlinear theory for multidimensional relativistic plasma wave wakefields," Physics of Plasmas, vol. 13, no. 5, p. 056709, 2006.

[31] C. D. Decker, W. B. Mori, K.-C. Tzeng, and T. Katsouleas, "The evolution of ultra-intense, short-pulse lasers in underdense plasmas," Physics of Plasmas, vol. 3, no. 5, pp. 2047-2056, 1996.

[32] M. Perry, "Multilayer dielectric gratings: Increasing the power of light," Lawrence Livermore National Laboratory Science $\&$ Technology Review, pp. 24-33, Sep 1995.

[33] K. Tanaka, T. Yabuuchi, T. Sato, R. Kodama, Y. Kitagawa, T. Takahashi, T. Ikeda, Y. Honda, and S. Okuda, "Calibration of imaging plate for high energy electron spectrometer," Rev. Sci. Instr., vol. 76, JAN 2005.

[34] K. BOCKASTEN, "Transformation of Observed Radiances into Radial Distribution of Emission of a Plasma," J. Opt. Soc. Am., vol. 51, no. 9, pp. 943-\&, 1961.

[35] V. Malka, C. Coulaud, J. Geindre, V. Lopez, Z. Najmudin, D. Neely, and F. Amiranoff, "Characterization of neutral density profile in a wide range of pressure of cylindrical pulsed gas jets," Rev. Sci. Instr., vol. 71, pp. 2329-2333, JUN 2000.

[36] J. E. Ralph, C. E. Clayton, F. Albert, B. B. Pollock, S. F. Martins, A. E. Pak, K. A. Marsh, J. L. Shaw, A. Till, J. P. Palastro, W. Lu, S. H. Glenzer, L. O. Silva, W. B. Mori, C. Joshi, and D. H. Froula, "Laser wakefield acceleration at reduced density in the self-guided regime," Phys. Plasmas, vol. 17, no. 5, p. $056709,2010$.

[37] B. B. Pollock, J. S. Ross, G. R. Tynan, L. Divol, S. H. Glenzer, V. Leurent, J. P. Palastro, J. E. Ralph, D. H. Froula, C. E. Clayton, K. A. Marsh, A. E. Pak, T. L. Wang, and C. Joshi, "Two-screen method for determining electron 
beam energy and deflection from laser wakefield acceleration," in Proceedings of the 2009 Particle Accelerator Conference, (Vancouver, Canada), May 04 May 082009.

[38] C. W. Siders, S. P. Le Blanc, D. Fisher, T. Tajima, M. C. Downer, A. Babine, A. Stepanov, and A. Sergeev, "Laser wakefield excitation and measurement by femtosecond longitudinal interferometry," Phys. Rev. Lett., vol. 76, pp. 35703573, May 1996.

[39] A. Popp, J. Osterhoff, Z. Major, R. Horlein, M. Fuchs, R. Weigngartner, J. Vieira, M. Marti, R. A. Fonseca, L. O. Silva, S. M. Hooker, F. Gruner, F. Krasz, and S. Karsh, "All-optical steering of laser-wakefield-accelerated electron beams," Phys. Rev. Lett. (submitted), 2010.

[40] S. F. Martins, R. A. Fonseca, W. Lu, W. B. Mori, and L. O. Silva, "Exploring laser-wakefield-accelerator regimes for near-term lasers using particle-in-cell simulation in lorentz-boosted frames," Nat Phys, vol. 6, pp. 311-316, 042010. 